\title{
Clinical aspects of irritable bowel syndrome, with a special focus on visceral hypersensitivity and intestinal permeability
}

Citation for published version (APA):

Ludidi, S. (2014). Clinical aspects of irritable bowel syndrome, with a special focus on visceral hypersensitivity and intestinal permeability. [Doctoral Thesis, Maastricht University]. Maastricht University. https://doi.org/10.26481/dis.20140903sl

Document status and date:

Published: 01/01/2014

DOI:

10.26481/dis.20140903sl

Document Version:

Publisher's PDF, also known as Version of record

Please check the document version of this publication:

- A submitted manuscript is the version of the article upon submission and before peer-review. There can be important differences between the submitted version and the official published version of record.

People interested in the research are advised to contact the author for the final version of the publication, or visit the DOI to the publisher's website.

- The final author version and the galley proof are versions of the publication after peer review.

- The final published version features the final layout of the paper including the volume, issue and page numbers.

Link to publication

\footnotetext{
General rights rights.

- You may freely distribute the URL identifying the publication in the public portal. please follow below link for the End User Agreement:

www.umlib.nl/taverne-license

Take down policy

If you believe that this document breaches copyright please contact us at:

repository@maastrichtuniversity.nl

providing details and we will investigate your claim.
}

Copyright and moral rights for the publications made accessible in the public portal are retained by the authors and/or other copyright owners and it is a condition of accessing publications that users recognise and abide by the legal requirements associated with these

- Users may download and print one copy of any publication from the public portal for the purpose of private study or research.

- You may not further distribute the material or use it for any profit-making activity or commercial gain

If the publication is distributed under the terms of Article $25 \mathrm{fa}$ of the Dutch Copyright Act, indicated by the "Taverne" license above, 
Clinical aspects of irritable bowel syndrome, with a special focus on visceral hypersensitivity and intestinal permeability 
(C) Copyright Samefko Ludidi, Maastricht 2014

ISBN: 978-90-9028436-1

Coverdesign: Stark Artworks

Layout: Tiny Wouters

Printing: Andi Druk

Printing of this thesis was financially supported by: Winclove Probiotics B.V., Nederlandse Vereniging voor Gastro-Enterologie, Ortho-Vision Holding B.V., Hotel Beaumont B.V., Harry's B.V., ABN-AMRO, Fysiostofberg B.V., Authentic Communication and Health Potential. 


\title{
Clinical aspects of irritable bowel syndrome, with a special focus on visceral hypersensitivity and intestinal permeability
}

\author{
PROEFSCHRIFT
}

ter verkrijging van de graad van doctor aan de Universiteit Maastricht, op gezag van de Rector Magnificus, Prof. dr. L.L.G. Soete volgens het besluit van het College van Decanen, in het openbaar te verdedigen op woensdag 3 september 2014 om 14.00 uur

door

Samefko Ludidi 


\section{Promotores}

Prof. dr. A.A.M. Masclee

\section{Copromotores}

Dr. J.M. Conchillo

Dr. D.M.A.E. Jonkers

\section{Beoordelingscommissie}

Prof. dr. F-J. van Schooten, voorzitter

Dr. J.W.M. Muris

Prof. dr. J.J. van Os

Prof. dr. A.J.P.M. Smout, AMC, Amsterdam

Dr. P.P. van der Veek, Medisch Centrum Haaglanden, Den Haag 
Aan mijn ouders 



\section{Contents}

$\begin{array}{lll}\text { Chapter } 1 \text { General introduction } & 9\end{array}$

Chapter 2 Does meal ingestion enhance sensitivity of visceroperception $\quad 27$ assessment in irritable bowel syndrome?

Neurogastroenterol Motil. 2012;24:47-53, e3

Chapter 3 Rectal hypersensitivity as hallmark for irritable bowel syndrome:

defining the optimal cut-off

Neurogastroenterol Motil. 2012;24:729-733, e345-346

Chapter $4 \quad$ Markers for visceral hypersensitivity in patients with

irritable bowel syndrome

Neurogastroenterology and Motility - Epub ahead of print

Chapter 5 Small intestinal permeability is increased in diarrhoea

predominant IBS, while alterations in gastroduodenal

permeability in all IBS subtypes are largely attributable to

confounders

Aliment Pharmacol and Ther - Epub ahead of print

Chapter 6 The intestinal barrier in irritable bowel syndrome:

subtype-specific effects of the systemic compartment

in an in vitro model

PLoS One - Provisionally accepted

Chapter $7 \quad$ Randomized clinical trial on the effect of a multispecies

probiotic on visceroperception in hypersensitive IBS patients

Neurogastroenterol Motil. 2014;26:705-714

Chapter 8 General discussion

Summary

Samenvatting

Dankwoord

Curriculum vitae

Scientific publications

Valorisation 




\title{
Chapter 1
}

\author{
General introduction
}


Chapter 1 


\section{Irritable bowel syndrome}

Irritable bowel syndrome (IBS) is a common functional disorder of the gastrointestinal (GI) tract, characterised by abdominal pain associated with an altered stool pattern, in the absence of apparent organic abnormalities ${ }^{1,2}$. The prevalence of IBS varies geographically with higher rates, i.e. up to $15 \%$, in Western countries ${ }^{3-5}$, indicating an association of the disorder with a Western lifestyle ${ }^{4}$. Furthermore, $50-70 \%$ of the total IBS population is female and disease onset peaks between the age of 25 and 55 years ${ }^{6}$.

In addition to the predominant symptoms (abdominal pain and altered bowel habits), IBS patients frequently report abdominal cramping, flatulence, bloating and dyspepsia. Non-GI symptoms such as headache, lower back pain and genitourinary symptoms are also more frequently reported among IBS patients, compared to healthy subjects ${ }^{4,7}$. It is apparent that IBS shows phenotypical heterogeneity and symptoms resemble those of other intestinal disorders such as inflammatory bowel disease, diverticular disease, diverticulitis and microscopic colitis ${ }^{8}$. Diagnosis therefore follows the exclusion of organic causes, and is based on the identification of symptoms according to the Rome III criteria for functional GI disorders ${ }^{1}$. The current Rome III Criteria define 4 subtypes, namely: IBS with either diarrhoea or constipation (IBS-D and IBS-C, respectively), IBS with a mixed stool pattern (IBS-M) and unspecified IBS (IBS-U).

Although IBS is (certainly) not a life-threatening disorder, it has clear impact on the patient's health-related quality of life (QoL). Studies have shown that symptoms form a major burden for IBS patients and affect physical and psychosocial functioning ${ }^{9-11}$, with a reduction in several domains of their QoL when compared to the general population, but also compared to patients suffering from other chronic conditions, (e.g. reflux disease, inflammatory bowel disease or diabetes) ${ }^{12}$. Low QoL in IBS patients has been attributed to somatic perceptions, e.g. pain and fatigue, as well as non-somatic perceptions, including a reduced perceived general health and dysfunctional cognitions $^{10,13}$. Furthermore, IBS is associated with increased use of health care services and high health care costs ${ }^{14-16}$. A study by Quigley et al. reported IBS to pose a significant economic burden to society in Europe, with costs estimated between $€ 700-€ 1600$ per patient a year ${ }^{17}$.

\section{Pathophysiology of IBS}

Although the exact pathophysiology is not known, several intestinal as well as central nervous system (brain) and psychosocial factors have a role in the development of IBS. At the gut level, altered visceral perception, reduced intestinal epithelial integrity ${ }^{18,19}$, 
low-grade inflammation ${ }^{3,20}$ and disturbed intestinal microbiota composition ${ }^{21}$ are more frequently observed in IBS. Furthermore, acute and chronic stress, anxiety, depression and dysfunctional cognitions have been reported to be more prevalent in patients with IBS compared to healthy controls ${ }^{13}$.

The pathophysiology of IBS is multifactorial and IBS comprises a wide phenotypical diversity. It is (certainly) not one single factor that characterises IBS and that is responsible for symptom generation; a complex interplay between several mechanisms occurs, resulting in many different IBS subtypes ${ }^{19,22,23}$. The following sections will further elaborate on (the more) several predominant pathophysiological mechanisms of IBS.

\section{Inflammation in IBS}

In the 1960s, Chaudhary and Truelove reported on an association between an episode of dysentery and self-reported symptom severity and showed that in approximately $25 \%$ of the patients with 'irritable colon syndrome', symptom onset occurred within a short interval after an enteric infection ${ }^{24}$. Subsequent studies confirmed a previous enteritis following Salmonella, Shigella or Campylobacter infections being a risk factor for what is now referred to as Post-infectious (PI-) IBS ${ }^{25-27}$. One of the largest studies on PI-IBS, the Walkerton cohort study, found an incidence of PI-IBS of $36.2 \%$ in 2069 subjects 2 years after the acute gastroenteritis (following water contamination with Escherichia coli and Campylobacter spp.) versus $10.1 \%$ in a control group s without gastroenteritis $^{25}$. Female sex ${ }^{3,28,29}$, duration of gastroenteritits, smoking ${ }^{30}$ and psychological distress and altered coping strategies prior to exposure ${ }^{3,4,28}$ were found to be risk factors for the development of PI-IBS.

The association with prior enteric infections, points to a role of intestinal inflammation in the development of IBS. Although organic causes should be excluded in the work-up for the diagnosis of IBS, over the years inflammation has gained interest as a putative pathophysiological mechanism. Hiatt \& Katz were among the first researchers that found increased numbers of mast cells in the muscularis propria layer of surgical colonic specimens of IBS patients ${ }^{31}$. To date, increasing evidence exists for a low-grade inflammation in IBS. Mucosal mast cell numbers were found to be increased in mucosal specimens of patients with IBS ${ }^{32,33}$. In addition, higher numbers of mast cells have been reported in close proximity to enteric nerve endings. Barbara et al., found mast cell infiltration in the colonic mucosa of about $75 \%$ of IBS patients, covering $9.2 \%$ of the mucosal area in IBS patients versus 3.3\% in healthy subjects. In these patients, mucosal mast cells numbers correlated significantly with abdominal pain scores ${ }^{34}$. It was also 
found that mast-cell activation. (i.e. degranulation and release of tryptase) and increased numbers of degranulating mast cells were present in IBS patients versus controls $^{34}$. Presently, findings are not uniform but in general, data point to a more pronounced increase in mast cells numbers and activation in patients with the IBS-D subtype s3,35-38. $^{3}$.

Furthermore, several studies have suggested an imbalanced systemic cytokine profile, potentially contributing to IBS pathophysiology ${ }^{39}$. Some studies only found minor differences or even no differences in plasma cytokine levels between IBS patients and healthy subjects ${ }^{40-42}$, but several groups have reported increased pro-inflammatory and/ or reduced anti-inflammatory plasma cytokine levels in IBS patients versus healthy subjects $^{40,43-45}$. In a review by Bashashati et al., increases were reported for IL- 6 and IL-8 and a decrease for $\mathrm{IL}-10^{39}$, In addition, genotypes encoding for a high TNF- $\alpha$ secretion $^{46,47}$ and a low IL-10 secretion ${ }^{47,48}$, were significantly more frequent in IBS patients compared to healthy controls.

\section{Intestinal permeability}

The intestinal barrier permits absorption of nutrients while preventing undesirable solutes, microbes, and antigens from entering the body. The intestinal barrier consist of a monolayer of epithelial cells covered by a mucus layer and the intestinal microbiota, and underneath supported by the presence of the local intestinal immune system.

The epithelial barrier is sealed by the intercellular apical junctional complex, consisting of tight junctions and adherens junctions, regulating paracellular permeability. Increased intestinal permeability is associated with several intestinal disease conditions, such as Inflammatory Bowel Diseases (IBD), but also systemic disorders such as diabetes mellitus (DM) type 1 and 2. Also in IBS, intestinal permeability was repeatedly found to be increased ${ }^{19,49-51}$. An altered barrier function is a more frequent phenomenon in patients with IBS-D and it has been reported that up to $40 \%$ of patients with IBS-D have an altered intestinal permeability ${ }^{19}$.

Recently, Bertieaux et al. found that there is also a subtype-dependent alteration in TJ protein expression and distribution in patients with IBS. In general, protein expression of Zonula-occludens (ZO)-1 and occludin were found to be reduced in IBS when compared to HC. Moreover, expression for occludin and claudin-1 lower in patients with IBS-D, but not in IBS-C and IBS-M subgroups ${ }^{51}$. Several biological substances can affect $\mathrm{TJ}$ functioning and integrity, including inflammatory mediators. Pro-inflammatory cytokines, such as interleukin(IL)-1, IL-3 and IL-4, tumor necrosis factor (TNF)-alpha and interferon (IF)-gamma, have been shown to reduce epithelial integrity in vitro ${ }^{52-54}$. 
In patients with IBS, proteases may also contribute to altered barrier function. Serine proteases, such as mast cell-derived tryptase, were found to be increased in the mucosa and faecal homogenates of IBS patients, especially in those with the diarrhoeapredominant subtype ${ }^{35-37}$. Gecse et al. found that inhibition of serine proteases prevented the increase in intestinal permeability in patients with IBS- $D^{35}$. Up to now, several research groups have confirmed that luminal exposure to tryptase results in an increased paracellular permeability employing in vitro and ex vivo models ${ }^{35,37,55}$. Most studies on potential mediators contributing to intestinal (hyper)permeability in IBS have focused on luminal factors (i.e. apical exposure). The potential role of systemic factors remains to be explored.

Finally, an altered intestinal microbiota composition may influence intestinal permeability in multiple ways. First, if the intestinal microbiota composition is altered, this could negatively affect competition with noxious entero-invading bacterial strains for epithelial colonisation sites, hence intestinal permeability. Second, interactions with the local enteric immune system e.g. via Toll-like receptors and subsequent signal transduction may affect permeability directly and indirectly. Third, the intestinal microbiota may influence epithelial tight junctions (e.g. rearrangement), hereby affecting intestinal permeability. Via changes in intestinal microbiota composition intestinal permeability may be affected in a negative way but potentially also in a positive way by re-enforcing the barrier.

\section{Altered visceral perception}

Visceral hypersensitivity has been observed in $30-70 \%$ of IBS patients, and is considered an important hallmark of the disorder (56-59). Hypersensitivity originally refers to increased sensations in response to somatic stimuli. To date, it also applies to sensations to visceral stimuli. In clinical practice, mechanical stimuli are represented by pressure- or volume increments applied to the intestinal lumen. In this respect, visceral hypersensitivity refers to an increased perception of mechanical stimuli applied to the intestinal lumen, perceived as discomfort and pain. Hypersensitivity in general comprises two components, i.e. allodynia and hyperalgesia. The former refers to an increased pain sensation in response to physiological stimuli (i.e. lower threshold for pain), whereas the latter refers to increased intensity of pain sensation in response to painful stimuli ${ }^{60,61}$.

The rectal barostat is a generally accepted and reproducible method to assess and quantify visceral perception ${ }^{62}$. It consists of a computer-driven pump that is able to inand deflate air into a polyethylene bag, which is placed inside the rectum, or elsewhere 
in the intestine. Distension protocols differ between institutes with regard to duration and type of inflation (tonic vs. phasic), pressure interval (ascending vs. randomized distension), and measurement of response magnitude with perceptual thresholds or ratings using Likert- or visual analogue scales respectively. This lack of standardisation may contribute to the varying prevalence of visceral hypersensitivity reported in IBS patients.

Although the exact relation between altered visceroperception and clinical outcomes in IBS is unclear ${ }^{63}$, IBS patients with enhanced visceroperception, not only show reduced thresholds for pain but also increased viscerosomatic referral in response to colonic or rectal distensions. Also, severity of self-reported somatic and psychological symptoms (e.g. pain, bloating, diarrhoea, anxiety, depression) have shown to be related to visceral hypersensitivity $^{59,64}$.

The exact causes of visceral hypersensitivity are unknown, but it is hypothesised that both peripheral and central mechanisms are involved in the perception of pain ${ }^{65}$. Postinflammatory hyperalgesia is a well-documented phenomenon ${ }^{66,67}$, and inflammationrelated sensitisation of the intestine could be an important local factor contributing to visceral hypersensitivity in $\mathrm{IBS}^{68}$. Local inflammation could give rise to altered barrier function, allowing both luminal contents and inflammatory mediators to trigger sensory afferent nerve endings ${ }^{69,70}$.

Evidence for this hypothesis was obtained by Zhou et al., showing visceral hypersensitivity in IBS-D patients with increased permeability ${ }^{19}$. Several animal studies indicate that the inflammatory mediators histamine and tryptase contribute to altered visceral perception by inducing hyperalgesia ${ }^{27,71,72}$. In addition, Gl pathogens or commensal intestinal bacteria are able to affect nociception by employing direct effects on visceral afferents or indirectly by modulating barrier function and/ or the immune system $^{73,74}$.

Finally, dysregulation of the enteric neuroendocrine system may contribute to visceral hypersensitivity as observed in a subgroup of $\mathrm{IBS}^{75}$. Increasing evidence suggests that serotonergic dysregulation may contribute to altered intestinal perception in patients with $\mathrm{IBS}^{76,77}$. In line with this, Cremon et al. demonstrated that the release of serotonin (5-hydroxyryptamine; 5 -HT) correlated with intensity of perceived abdominal pain in patients with IBS (78). Upon its release from enterochromaffin (EC) cells, 5-HT excites extrinsic afferent neurons via $5-\mathrm{HT} 3$ receptors. Sensory input is subsequently transferred via the dorsal root ganglion of the spinal cord to be processed in the CNS. When compared to HC, IBS patients have higher mucosal serotonin levels ${ }^{29,78}$. An excess of 5-HT in plasma of PI-IBS and IBS-D, but contrarily also abnormally low 5-HT availability in IBS-C plasma has been observed ${ }^{79}$. To date, inconsistencies exist with 
respect to 5-HT levels in IBS and how this relates to IBS-subtypes. Here, a lower serotonin reuptake transporter (SERT) expression in patients with IBS-D particularly may be involved ${ }^{80,81}$.

Apart from local alterations, central changes such as increased brain activity can occur in response to increased peripheral sensory input ${ }^{82}$. Negative emotions and stress, frequently occurring among IBS patients, could also affect perception to viscerally applied stimuli ${ }^{41}$. As a result, cortical processes, such as vigilance, anticipation and increased symptom-directed anxiety could explain the increased tendency to report early pain in $\mathrm{IBS}^{65,83}$.

Several studies have shown that visceroperception correlates with symptom severity in patients with IBS and these patients with visceral hypersensitivity may be regarded as a distinct subgroup, regardless of bowel habit. Can visceroperception be considered as biomarker for IBS and IBS symptoms? Do changes in viscerperception by (therapeutic) interventions reflect or predict changes in IBS symptoms and should the barostat test become a standard instrument in selection of IBS patients for therapeutic intervention trials? These question need to be answered in the near future

\section{Psychosocial factors}

Apart from the possible involvement of several physiological mechanistic factors, IBS is also considered a bio-psychosocial disorder ${ }^{84-86}$. Neuroticism, anxiety, depression and dysfunctional cognitions have been reported to be more prevalent in IBS ${ }^{13,87-89}$. These factors and other psychosocial factors (e.g. chronic stress, abuse history or childhood trauma) may induce or aggravate symptoms ${ }^{85}$. For instance, Thijssen et al. showed that the presence of anxiety or depressive disorders as well as dysfunctional cognitions significantly affect symptom severity in patients with IBS $^{13}$. Also, at the level of visceral sensitivity it has been demonstrated that patients with IBS and a history of abuse had significantly lower thresholds for pain and urge and a greater tendency to report pain in response to rectal distensions versus IBS patients without history of abuse ${ }^{90}$.

\section{Treatment of IBS}

Because of the diversity of symptoms and varying stool patterns in patients with IBS, pharmacological and other treatment strategies and effects vary widely. In general, treatment focuses on those symptoms - often predominant bowel habits - that have largest impact on the patients' QoL. 
Although many treatment approaches have been investigated in the past decades, only few have shown to be effective. A recent Cochrane review by Ruepert et al. summarises recent findings and has shown that there is no clear evidence that bulking agents are effective as treatment option for IBS. Antispasmodics on the contrary, were found to have proven efficacy. Especially cimetropium/ diclomine ${ }^{91-93}$, peppermint oil $^{94,95}$, pinaverium $^{96}$ and trimebutine ${ }^{97}$ have shown to be effective in improving pain scores, overall IBS symptom scores. Although effectiveness may vary with individual patient features, anti-depressants (i.e. tricylic acids and selective serotonin reuptake inhibitors) have also shown to be beneficial for the reduction of pain ${ }^{98,99}$ and overall IBS symptoms $^{99,100}$.

Also, psychosocial-directed therapies and mind-body directed interventions may help IBS patients in managing symptoms. Having modest but proven efficacy, mindfulness, meditation, relaxation

training and hypnotherapy are the most commonly employed psychological interventions ${ }^{101,102}$. Implementation of such therapies in daily practice however, may be difficult. Patients with IBS have alternating symptom presentation and tend to seek specialised help only when symptoms are present. Compliance to therapy may be low as a result of high drop-out rates, especially in these psychosocial-directed therapies. This is partly explained by the alternating disease state of IBS: patients typically seek medical care when symptoms are at worst, if symptoms are not present, patients have the tendency to restrain from medical help.

IBS patients commonly report symptom aggravation after food intake, such as dairy, caffeine, alcohol or foods high in carbohydrates and/ or fats ${ }^{103}$. While food serves as a trigger, food products may also provide symptom relief. Böhn et al. found lower meat intake and higher fruit and vegetable intake among IBS patients ${ }^{104}$. These dietary habits showed no vast differences in overall nutrient intake between IBS patients and healthy subjects $^{104}$ and previous research shows that avoidance of food items among IBS patients had no effects on body weight ${ }^{103}$. Interest in dietary interventions for IBS patients is increasing. Diets low in fermentable oligo- di- monosaccharide and polyols (FODMAPS) have gained interest as they may have reduce bacterial fermentation and gas production, and hence symptom reduction in IBS. A recent study by de Roest et al. has shown that a low FODMAP diet contributed to symptom improvement of abdominal pain, bloating, flatulence and diarrhoea in patients with IBS after 15 months of follow-up ${ }^{105}$.

The increasing interest in the role of the intestinal microbiota, resulted in several probiotic intervention studies. Probiotics are reported to affect the intestinal microbiota and, immune function, but may also improve intestinal barrier and even 
visceral perception ${ }^{106}$. Recently, it has been reported that overall, probiotics have beneficial effect when compared to placebo ${ }^{107}$. However, in general effects are only modest, which might (partly) be due to the heterogonous group of patients included and studies are difficult to compare because of differences in probiotic products given and study designs and intention-to treat analyses are often lacking ${ }^{107,108}$.

Further developments in understanding the complex interactions along the brain-gut axis, taking into account the disease heterogeneity may lead to a better understanding and more insight in pathophysiology and symptom development. A next step includes expansion of knowledge and development of quality instruments to evaluate pharmacological, dietary, prebiotic/ probiotic, metabolic and psychological therapeutic interventions intended to provide relief of symptoms in IBS ${ }^{109,110}$.

\section{Aims and outline of investigations in this thesis}

IBS is a highly prevalent functional disorder with a heterogeneous phenotype and the complex multifactorial pathophysiology, which seems to differ between subgroups of patients.

Visceral hypersensitivity is regarded a key factor and hallmark and potential biomarker of IBS. We aimed to optimise and standardise the diagnostic procedures for visceral hypersensitivity in patients with IBS, to investigate putative underlying mechanisms and to target and thereafter treat a subgroup of IBS patients with visceral hypersensitivity with a specifically designed multispecies probiotic.

As food intake may trigger symptoms in patients with IBS, we assessed whether a standardised meal would help to enhance sensitivity of the rectal barostat procedure in detecting visceral hypersensitivity in IBS and discriminating IBS patients from healthy controls (chapter 2). Subsequently, in chapter 3 optimising the cut-off for assessing visceral hypersensitivity with the rectal barostat has been described.

Since visceral hypersensitivity is frequently observed in IBS patients, though not in all patients, we questioned in chapter 4 which biological and clinical features could further characterise the hypersensitive versus the normosensitive patients. One potential mechanism associated with IBS is an increased intestinal permeability, but studies in large well-defined cohorts are scarce. The in vivo intestinal permeability for proximal GI tract, small intestine and large intestine was therefore assessed with a newly validated multi-sugar in a large group of 91 well phenotyped IBS patients as well as healthy controls (chapter 5). Although altered intestinal permeability is generally considered to be affected by intestinal factors, in chapter 6 we took another route and investigated whether the central compartment with circulating mediators may have a role in altered permeability in different IBS subtypes. For this purpose we used an in vitro model with 
cultured intestinal cells in 3D structure. Finally in chapter 7, an intervention is performed in which we aimed to reduce visceral hypersensitivity using a multifactorial approach targeting visceroperception itself, intestinal permeability, inflammation with a specifically designed multispecies probiotic. Chapter 8 summarises the main findings of all studies ending up with a discussion and implications for future research. 


\section{References}

1. Longstreth GF, Thompson WG, Chey WD, Houghton LA, Mearin F, Spiller RC. Functional bowel disorders. Gastroenterology. 2006;130:1480-91.

2. Thompson WG. Irritable bowel syndrome: a management strategy. Baillieres Best Pract Res Clin Gastroenterol. 1999;13:453-60..

3. Spiller R, Garsed K. Infection, inflammation, and the irritable bowel syndrome. Dig Lliver Dis. 2009;41:844-9.

4. Gwee KA, Wee S, Wong ML, Png DJ. The prevalence, symptom characteristics, and impact of irritable bowel syndrome in an asian urban community. Am J Gastroenterol. 2004;99: 924-31.

5. Xiong LS, Chen MH, Chen HX, Xu AG, Wang WA, Hu PJ. A population-based epidemiologic study of irritable bowel syndrome in South China: stratified randomized study by cluster sampling. Aliment Pharmacol Ther. 2004;19:1217-24.

6. Hungin AP, Chang L, Locke GR, Dennis EH, Barghout V. Irritable bowel syndrome in the United States: prevalence, symptom patterns and impact. Aliment Pharmacol Ther. 2005;21:1365-75.

7. Heitkemper MM, Jarrett ME. Update on irritable bowel syndrome and gender differences. Nutr Clin Pract. 2008;23:275-83.

8. Spiegel BM, Gralnek IM, Bolus R, Chang L, Dulai GS, Naliboff B, Mayer EA. Is a negative colonoscopy associated with reassurance or improved health-related quality of life in irritable bowel syndrome? Gastrointest Endosc. 2005;62:892-9.

9. El-Serag HB, Olden K, Bjorkman D. Health-related quality of life among persons with irritable bowel syndrome: a systematic review. Aliment Pharmacol Ther. 2002;16:1171-85.

10. Gralnek IM, Hays RD, Kilbourne A, Naliboff B, Mayer EA. The impact of irritable bowel syndrome on health-related quality of life. Gastroenterology. 2000;119:654-60.

11. Gulewitsch MD, Enck P, Hautzinger M, Schlarb AA. Irritable bowel syndrome symptoms among German students: prevalence, characteristics, and associations to somatic complaints, sleep, quality of life, and childhood abdominal pain. European journal of gastroenterology \& hepatology. 2011 Apr;23(4):311-6.

12. Monnikes H. Quality of life in patients with irritable bowel syndrome. J Clin Gastroenterol. 2011;45 Suppl:S98-101.

13. Thijssen AY, Jonkers DM, Leue C, van der Veek PP, Vidakovic-Vukic M, van Rood YR, Clemens $C H$, Masclee AA. Dysfunctional cognitions, anxiety and depression in irritable bowel syndrome. J Clin Gastroenterol. 2010;44:e236-41.

14. Brun-Strang C, Dapoigny M, Lafuma A, Wainsten JP, Fagnani F. Irritable bowel syndrome in France: quality of life, medical management, and costs: the Encoli study. Eur J Gastroenterol Hepatol. 2007;19: 1097-103.

15. Hillila MT, Farkkila NJ, Farkkila MA. Societal costs for irritable bowel syndrome--a population based study. Scand J Gastroenterol. 2010;45:582-91.

16. Simren M, Brazier J, Coremans G, Dapoigny M, Muller-Lissner SA, Pace F, Smout AJ, Stockbrugger RW, Vatn $\mathrm{MH}$, Whorwell PJ. Quality of life and illness costs in irritable bowel syndrome. Digestion. 2004;69:254-61.

17. Quigley EM, Bytzer P, Jones R, Mearin F. Irritable bowel syndrome: the burden and unmet needs in Europe. Dig Liver Dis. 2006;38:717-23.

18. Rao AS, Camilleri M, Eckert DJ, Busciglio I, Burton DD, Ryks M, Wong BS, Lamsam J, Singh R, Zinsmeister AR. Urine sugars for in vivo gut permeability: validation and comparisons in irritable bowel syndromediarrhea and controls. Am J Physiol Gastrointest Liver Physiol. 2011;301:G919-28.

19. Zhou $Q$, Zhang B, Verne GN. Intestinal membrane permeability and hypersensitivity in the irritable bowel syndrome. Pain. 2009;146:41-6.

20. Ohman L, Simren M. Pathogenesis of IBS: role of inflammation, immunity and neuroimmune interactions. Nat Rev Gastroenterol Hepatol. 2010;7:163-73.

21. Ohman L, Simren M. Intestinal microbiota and its role in irritable bowel syndrome (IBS). Curr Gastroenterol Rep. 2013;15:323. 
22. Piche T, Barbara G, Aubert P, Bruley des Varannes S, Dainese R, Nano JL, Cremon C, Stanghellini V, De Giorgio R, Galmiche JP, Neunlist M. Impaired intestinal barrier integrity in the colon of patients with irritable bowel syndrome: involvement of soluble mediators. Gut. 2009;58:196-201.

23. Yu YB, Zuo XL, Zhao QJ, Chen FX, Yang J, Dong YY, Wang P, Li YQ. Brain-derived neurotrophic factor contributes to abdominal pain in irritable bowel syndrome. Gut. 2012;61:685-94.

24. Chaudhary NA, Truelove SC. The irritable colon syndrome. A study of the clinical features, predisposing causes, and prognosis in 130 cases. Q J Med. 1962;31:307-22.

25. Marshall JK. Post-infectious irritable bowel syndrome following water contamination. Kidney lint Suppl. 2009;(112):S42-3.

26. McKendrick MW, Read NW. Irritable bowel syndrome--post salmonella infection. J Infect. 1994;29:1-3.

27. Spiller RC, Jenkins D, Thornley JP, Hebden JM, Wright T, Skinner M, Neal KR. Increased rectal mucosal enteroendocrine cells, T lymphocytes, and increased gut permeability following acute Campylobacter enteritis and in post-dysenteric irritable bowel syndrome. Gut. 2000;47:804-11.

28. Barbara G, De Giorgio R, Stanghellini V, Cremon C, Corinaldesi R. A role for inflammation in irritable bowel syndrome? Gut. 2002;51 Suppl 1:i41-4.

29. Dunlop SP, Jenkins D, Spiller RC. Distinctive clinical, psychological, and histological features of postinfective irritable bowel syndrome. Am J Gastroenterol. 2003;98:1578-83.

30. Parry S, Forgacs I. Intestinal infection and irritable bowel syndrome. Eur J Gastroenterol Hepatol. 2005; 17:5-9.

31. Hiatt RB, Katz L. Mast cells in inflammatory conditions of the gastrointestinal tract. Am J Gastroenterol. 1962;37:541-5.

32. Wang LH, Fang XC, Pan GZ. Bacillary dysentery as a causative factor of irritable bowel syndrome and its pathogenesis. Gut. 2004;53:1096-101.

33. Lee H, Park JH, Park DI, Kim HJ, Cho YK, Sohn Cl, Jeon WK, Kim BI, Chae SW. Mucosal mast cell count is associated with intestinal permeability in patients with diarrhea predominant irritable bowel syndrome. J Neurogastroenterol Motil. 2013;19:244-50.

34. Barbara G, Stanghellini V, De Giorgio R, Cremon C, Cottrell GS, Santini D, Pasquinelli G, Morselli-Labate AM, Grady EF, Bunnett NW, Collins SM, Corinaldesi R. Activated mast cells in proximity to colonic nerves correlate with abdominal pain in irritable bowel syndrome. Gastroenterology. 2004;126: 693-702.

35. Gecse K, Roka R, Ferrier L, Leveque M, Eutamene H, Cartier C, Ait-Belgnaoui A, Rosztoczy A, Izbeki F, Fioramonti J, Wittmann T, Bueno L. Increased faecal serine protease activity in diarrhoeic IBS patients: a colonic lumenal factor impairing colonic permeability and sensitivity. Gut. 2008;57:591-9.

36. Lee JW, Park JH, Park DI, Park JH, Kim HJ, Cho YK, Sohn Cl, Jeon WK, Kim BI. Subjects with diarrheapredominant IBS have increased rectal permeability responsive to tryptase. Dig Dis Sci. 2010;55:2922-8.

37. Annahazi A, Ferrier L, Bezirard V, Leveque $M$, Eutamene $H$, Ait-Belgnaoui $A$, Coeffier M, Ducrotte $P$, Roka R, Inczefi O, Gecse K, Rosztoczy A, Molnar T, Ringel-Kulka T, Ringel Y, Piche T, Theodorou V, Wittmann T, Bueno L. Luminal Cysteine-Proteases Degrade Colonic Tight Junction Structure and Are Responsible for Abdominal Pain in Constipation-Predominant IBS. Am J Gastroenterol. 2013;108: 1322-31.

38. Lee KJ, Kim YB, Kim JH, Kwon HC, Kim DK, Cho SW. The alteration of enterochromaffin cell, mast cell, and lamina propria T lymphocyte numbers in irritable bowel syndrome and its relationship with psychological factors. J Gastroenterol Hepatol. 2008;23:1689-94.

39. Bashashati M, Rezaei N, Andrews CN, Chen CQ, Daryani NE, Sharkey KA, Storr MA. Cytokines and irritable bowel syndrome: where do we stand? Cytokine. 2012;57:201-9.

40. Dinan TG, Quigley EM, Ahmed SM, Scully P, O'Brien S, O'Mahony L, O'Mahony S, Shanahan F, Keeling PW. Hypothalamic-pituitary-gut axis dysregulation in irritable bowel syndrome: plasma cytokines as a potential biomarker? Gastroenterology. 2006;130:304-11.

41. Elsenbruch S, Holtmann G, Oezcan D, Lysson A, Janssen O, Goebel MU, Schedlowski M. Are there alterations of neuroendocrine and cellular immune responses to nutrients in women with irritable bowel syndrome? Am J Gastroenterol. 2004;99:703-10.

42. McKernan DP, Gaszner G, Quigley EM, Cryan JF, Dinan TG. Altered peripheral toll-like receptor responses in the irritable bowel syndrome. Aliment Pharmacol Ther. 2011;33:1045-52. 
43. Ohman L, Isaksson S, Lindmark AC, Posserud I, Stotzer PO, Strid H, Sjovall H, Simren M. T-cell activation in patients with irritable bowel syndrome. Am J Gastroenterol. 2009;104:1205-12.

44. Scully P, McKernan DP, Keohane J, Groeger D, Shanahan F, Dinan TG, Quigley EM. Plasma cytokine profiles in females with irritable bowel syndrome and extra-intestinal co-morbidity. Am J Gastroenterol. 2010 Oct;105(10):2235-43.

45. Hua MC, Lai MW, Kuo ML, Yao TC, Huang JL, Chen SM. Decreased interleukin-10 secretion by peripheral blood mononuclear cells in children with irritable bowel syndrome. J Pediatric Gastroenterol Nutr. 2011;52:376-81.

46. Barkhordari E, Rezaei N, Mahmoudi M, Larki P, Ahmadi-Ashtiani HR, Ansaripour B, Alighardashi M, Bashashati M, Amirzargar AA, Ebrahimi-Daryani N. T-helper 1, T-helper 2, and T-regulatory cytokines gene polymorphisms in irritable bowel syndrome. Inflammation. 2010;33:281-6.

47. van der Veek PP, van den Berg M, de Kroon YE, Verspaget HW, Masclee AA. Role of tumor necrosis factor-alpha and interleukin-10 gene polymorphisms in irritable bowel syndrome. Am J Gastroenterol. 2005;100:2510-6.

48. Lee HJ, Lee SY, Choi JE, Kim JH, Sung IK, Park HS, Jin CJ. G protein beta3 subunit, interleukin-10, and tumor necrosis factor-alpha gene polymorphisms in Koreans with irritable bowel syndrome. Neurogastroenterol Motil. 2010;22:758-63.

49. Dunlop SP, Hebden J, Campbell E, Naesdal J, Olbe L, Perkins AC, Spiller RC. Abnormal intestinal permeability in subgroups of diarrhea-predominant irritable bowel syndromes. Am J Gastroenterol. 2006;101:1288-94.

50. Marshall JK, Thabane M, Garg AX, Clark W, Meddings J, Collins SM, Investigators WEL. Intestinal permeability in patients with irritable bowel syndrome after a waterborne outbreak of acute gastroenteritis in Walkerton, Ontario. Aliment Pharmacol Ther. 2004;20:1317-22.

51. Bertiaux-Vandaele N, Youmba SB, Belmonte L, Lecleire S, Antonietti M, Gourcerol G, Leroi AM, Dechelotte $\mathrm{P}$, Menard JF, Ducrotte $\mathrm{P}$, Coeffier $\mathrm{M}$. The expression and the cellular distribution of the tight junction proteins are altered in irritable bowel syndrome patients with differences according to the disease subtype. Am J Gastroenterol. 2011;106:2165-73.

52. Ahdieh $M$, Vandenbos $T$, Youakim A. Lung epithelial barrier function and wound healing are decreased by IL-4 and IL-13 and enhanced by IFN-gamma. Am J Physiol Cell Physiol. 2001;281:C2029-38.

53. Oshima T, Laroux FS, Coe LL, Morise Z, Kawachi S, Bauer P, Grisham MB, Specian RD, Carter P, Jennings S, Granger DN, Joh T, Alexander JS. Interferon-gamma and interleukin-10 reciprocally regulate endothelial junction integrity and barrier function. Microvasc Res. 2001;61:130-43.

54. Youakim A, Ahdieh M. Interferon-gamma decreases barrier function in T84 cells by reducing ZO-1 levels and disrupting apical actin. Am J Physiol. 1999;276:G1279-88.

55. Jacob C, Yang PC, Darmoul D, Amadesi S, Saito T, Cottrell GS, Coelho AM, Singh P, Grady EF, Perdue M, Bunnett NW. Mast cell tryptase controls paracellular permeability of the intestine. Role of proteaseactivated receptor 2 and beta-arrestins. J Biol Chem. 2005;280:31936-48.

56. Azpiroz F. Hypersensitivity in functional gastrointestinal disorders. Gut. 2002;51 Suppl 1:i25-8.

57. Azpiroz F, Bouin M, Camilleri M, Mayer EA, Poitras P, Serra J, Spiller RC. Mechanisms of hypersensitivity in IBS and functional disorders. Neurogastroenterol Motil. 2007;19(1 Suppl):62-88.

58. Ludidi S, Conchillo JM, Keszthelyi D, Van Avesaat M, Kruimel JW, Jonkers DM, Masclee AA. Rectal hypersensitivity as hallmark for irritable bowel syndrome: defining the optimal cutoff. Neurogastroenterol Motil. 2012;24:729-e346.

59. Posserud I, Syrous A, Lindstrom L, Tack J, Abrahamsson H, Simren M. Altered rectal perception in irritable bowel syndrome is associated with symptom severity. Gastroenterology. 2007;133:1113-23.

60. Camilleri M. Testing the sensitivity hypothesis in practice: tools and methods, assumptions and pitfalls. Gut. 2002;51 Suppl 1:i34-40.

61. Eijkelkamp N, Heijnen CJ, Carbajal AG, Willemen HL, Wang H, Minett MS, Wood JN, Schedlowski M, Dantzer R, Kelley KW, Kavelaars A. G protein-coupled receptor kinase 6 acts as a critical regulator of cytokine-induced hyperalgesia by promoting phosphatidylinositol 3-kinase and inhibiting p38 signaling. Mol Med. 2012;18:556-64.

62. Hammer HF, Phillips SF, Camilleri M, Hanson RB. Rectal tone, distensibility, and perception: reproducibility and response to different distensions. Am J Physiol. 1998;274:G584-90. 
63. Piche $M$, Arsenault $M$, Poitras $P$, Rainville $P$, Bouin $M$. Widespread hypersensitivity is related to altered pain inhibition processes in irritable bowel syndrome. Pain. 2010;148:49-58.

64. van der Veek PP, Van Rood YR, Masclee AA. Symptom severity but not psychopathology predicts visceral hypersensitivity in irritable bowel syndrome. Clin Gastroenterol Hepatol. 2008;6:321-8.

65. Keszthelyi D, Troost FJ, Simren M, Ludidi S, Kruimel JW, Conchillo JM, Masclee AA. Revisiting concepts of visceral nociception in irritable bowel syndrome. Eur J Pain. 2012 Apr 13.

66. Hughes PA, Brierley SM, Blackshaw LA. Post-inflammatory modification of colonic afferent mechanosensitivity. Clin Exp Pharmacol Physiol. 2009;36:1034-40.

67. Vergnolle N. Visceral afferents: what role in post-inflammatory pain? Auton Neurosci. 2010;153:79-83.

68. Mayer EA, Gebhart GF. Basic and clinical aspects of visceral hyperalgesia. Gastroenterology. 1994;107:271-93.

69. Dai C, Guandalini S, Zhao DH, Jiang M. Antinociceptive effect of VSL\#3 on visceral hypersensitivity in a rat model of irritable bowel syndrome: a possible action through nitric oxide pathway and enhance barrier function. Mol Cell Biochem. 2012;362:43-53.

70. Zhou Q, Souba WW, Croce CM, Verne GN. MicroRNA-29a regulates intestinal membrane permeability in patients with irritable bowel syndrome. Gut. 2010;59:775-84.

71. Barbara G, Wang B, Stanghellini V, de Giorgio R, Cremon C, Di Nardo G, Trevisani M, Campi B, Geppetti P, Tonini M, Bunnett NW, Grundy D, Corinaldesi R. Mast cell-dependent excitation of visceralnociceptive sensory neurons in irritable bowel syndrome. Gastroenterology. 2007;132:26-37.

72. Bueno L, Fioramonti J, Delvaux M, Frexinos J. Mediators and pharmacology of visceral sensitivity: from basic to clinical investigations. Gastroenterology. 1997;112:1714-43.

73. Al-Khatib K, Lin HC. Immune activation and gut microbes in irritable bowel syndrome. Gut Liver. 2009;3:14-9.

74. Rescigno M. The intestinal epithelial barrier in the control of homeostasis and immunity. Trends Immunol. 2011;32:256-64.

75. Zhou Q, Verne GN. New insights into visceral hypersensitivity--clinical implications in IBS. Nature reviews Gastroenterol Hepatol. 2011;8:349-55.

76. Labus JS, Mayer EA, Jarcho J, Kilpatrick LA, Kilkens TO, Evers EA, Backes WH, Brummer RJ, van Nieuwenhoven MA. Acute tryptophan depletion alters the effective connectivity of emotional arousal circuitry during visceral stimuli in healthy women. Gut. 2011;60:1196-203.

77. Spiller R, Bennett A. Searching for the answer to irritable bowel syndrome in the colonic mucosa: SERTainty and unSERTainty. Gastroenterology. 2007;132:437-41.

78. Cremon C, Carini G, Wang B, Vasina V, Cogliandro RF, De Giorgio R, Stanghellini V, Grundy D, Tonini M, De Ponti F, Corinaldesi R, Barbara G. Intestinal serotonin release, sensory neuron activation, and abdominal pain in irritable bowel syndrome. Am J Gastroenterol. 2011;106:1290-8.

79. Spiller R. Serotonergic agents and the irritable bowel syndrome: what goes wrong? Curr Opin Pharmacol. 2008;8:709-14.

80. Foley S, Garsed K, Singh G, Duroudier NP, Swan C, Hall IP, Zaitoun A, Bennett A, Marsden C, Holmes G, Walls A, Spiller RC. Impaired uptake of serotonin by platelets from patients with irritable bowel syndrome correlates with duodenal immune activation. Gastroenterology. 2011;140:1434-43 e1.

81. Spiller R. Serotonin and GI clinical disorders. Neuropharmacology. 2008;55:1072-80.

82. Wilder-Smith $\mathrm{CH}$, Schindler D, Lovblad K, Redmond SM, Nirkko A. Brain functional magnetic resonance imaging of rectal pain and activation of endogenous inhibitory mechanisms in irritable bowel syndrome patient subgroups and healthy controls. Gut. 2004;53:1595-601.

83. Dorn SD, Palsson OS, Thiwan SI, Kanazawa M, Clark WC, van Tilburg MA, Drossman DA, Scarlett Y, Levy RL, Ringel Y, Crowell MD, Olden KW, Whitehead WE. Increased colonic pain sensitivity in irritable bowel syndrome is the result of an increased tendency to report pain rather than increased neurosensory sensitivity. Gut. 2007;56:1202-9.

84. Drossman DA. Presidential address: Gastrointestinal illness and the biopsychosocial model. Psychosom Med. 1998;60:258-67.

85. Fichna J, Storr MA. Brain-Gut Interactions in IBS. Front Pharmacol. 2012;3:127.

86. Mach T. The brain-gut axis in irritable bowel syndrome--clinical aspects. Med Sci Monit. 2004;10: RA125-31. 
87. Chitkara DK, van Tilburg MA, Blois-Martin N, Whitehead WE. Early life risk factors that contribute to irritable bowel syndrome in adults: a systematic review. Am J Gastroenterol. 2008;103:765-74.

88. Levy RL, Olden KW, Naliboff BD, Bradley LA, Francisconi C, Drossman DA, Creed F. Psychosocial aspects of the functional gastrointestinal disorders. Gastroenterology. 2006;130:1447-58.

89. Whitehead WE, Burnett CK, Cook EW, 3rd, Taub E. Impact of irritable bowel syndrome on quality of life. Dig Dis Sci. 1996;41:2248-53.

90. Ringel Y, Drossman DA, Leserman JL, Suyenobu BY, Wilber K, Lin W, Whitehead WE, Naliboff BD, Berman S, Mayer EA. Effect of abuse history on pain reports and brain responses to aversive visceral stimulation: an FMRI study. Gastroenterology. 2008;134:396-404.

91. Centonze V, Imbimbo BP, Campanozzi F, Attolini E, Daniotti S, Albano O. Oral cimetropium bromide, a new antimuscarinic drug, for long-term treatment of irritable bowel syndrome. Am J Gastroenterol. 1988;83:1262-6.

92. Dobrilla G, Imbimbo BP, Piazzi L, Bensi G. Longterm treatment of irritable bowel syndrome with cimetropium bromide: a double blind placebo controlled clinical trial. Gut. 1990;31:355-8.

93. Page JG, Dirnberger GM. Treatment of the irritable bowel syndrome with Bentyl (dicyclomine hydrochloride). J Clin Gastroenterol. 1981;3:153-6.

94. Cappello G, Spezzaferro M, Grossi L, Manzoli L, Marzio L. Peppermint oil (Mintoil) in the treatment of irritable bowel syndrome: a prospective double blind placebo-controlled randomized trial. Dig Liver Dis. 2007;39:530-6.

95. Lech $\mathrm{Y}$, Olesen KM, Hey H, Rask-Pedersen E, Vilien M, Ostergaard O. [Treatment of irritable bowel syndrome with peppermint oil. A double-blind study with a placebo]. Ugeskr Laeger. 1988;150:2388-9.

96. Awad R, Dibildox M, Ortiz F. Irritable bowel syndrome treatment using pinaverium bromide as a calcium channel blocker. A randomized double-blind placebo-controlled trial. Acta Gastroenterol Latinoam. 1995;25:137-44.

97. Fielding JF. Double blind trial of trimebutine in the irritable bowel syndrome. Ir Med J. 1980;73: 377-9.

98. Drossman DA, Ringel Y, Vogt BA, Leserman J, Lin W, Smith JK, Whitehead W. Alterations of brain activity associated with resolution of emotional distress and pain in a case of severe irritable bowel syndrome. Gastroenterology. 2003;124:754-61.

99. Vahedi H, Merat S, Momtahen S, Kazzazi AS, Ghaffari N, Olfati G, Malekzadeh R. Clinical trial: the effect of amitriptyline in patients with diarrhoea-predominant irritable bowel syndrome. Aliment Pharmacol Ther. 2008;27:678-84.

100. Bahar RJ, Collins BS, Steinmetz B, Ament ME. Double-blind placebo-controlled trial of amitriptyline for the treatment of irritable bowel syndrome in adolescents. J Pediatr. 2008;152:685-9.

101. Pajak R, Lackner J, Kamboj SK. A systematic review of minimal-contact psychological treatments for symptom management in irritable bowel syndrome. J Psychosomatic Res. 2013;75:103-12.

102. Zijdenbos IL, de Wit NJ, van der Heijden GJ, Rubin G, Quartero AO. Psychological treatments for the management of irritable bowel syndrome. The Cochrane database of systematic reviews. 2009(1):CD006442.

103. Simren M, Mansson A, Langkilde AM, Svedlund J, Abrahamsson H, Bengtsson U, Bjornsson ES. Foodrelated gastrointestinal symptoms in the irritable bowel syndrome. Digestion. 2001;63:108-15.

104. Bohn L, Storsrud S, Simren M. Nutrient intake in patients with irritable bowel syndrome compared with the general population. Neurogastroenterol Motil. 2013;25:23-30 e1.

105. de Roest RH, Dobbs BR, Chapman BA, Batman B, O'Brien LA, Leeper JA, Hebblethwaite CR, Gearry RB. The low FODMAP diet improves gastrointestinal symptoms in patients with irritable bowel syndrome: a prospective study. Int J Clin Pract. 2013;67:895-903.

106. Hoveyda N, Heneghan C, Mahtani KR, Perera R, Roberts N, Glasziou P. A systematic review and metaanalysis: probiotics in the treatment of irritable bowel syndrome. BMC gastroenterology. 2009;9:15.

107. Moayyedi P, Ford AC, Talley NJ, Cremonini F, Foxx-Orenstein AE, Brandt LJ, Quigley EM. The efficacy of probiotics in the treatment of irritable bowel syndrome: a systematic review. Gut. 2010;59:325-32.

108. Simren M, Barbara G, Flint HJ, Spiegel BM, Spiller RC, Vanner S, Verdu EF, Whorwell PJ, Zoetendal EG, Rome Foundation $C$. Intestinal microbiota in functional bowel disorders: a Rome foundation report. Gut. 2013;62:159-76. 
109. Camilleri M. Review article: new receptor targets for medical therapy in irritable bowel syndrome. Aliment Pharmacol Ther. 2010;31:35-46.

110. Camilleri M, Katzka DA. Irritable bowel syndrome: methods, mechanisms, and pathophysiology. Genetic epidemiology and pharmacogenetics in irritable bowel syndrome. Am J Physiol Gastrointest Liver Physiol. 2012;302:G1075-84. 


\section{Chapter}

Does meal ingestion enhance sensitivity of visceroperception assessment in irritable bowel syndrome?

S Ludidi, J Conchillo, D Keszthelyi, C Koning, S Vanhoutvin, P Lindsey, A Leufkens, J Kruimel, D Jonkers, A Masclee

Neurogastroenterol Motil. 2012;24:47-53, e3. 


\section{Abstract}

\section{Background}

Visceral hypersensitivity is frequently observed in irritable bowel syndrome (IBS). Previous studies have shown that administration of a meal can aggravate symptoms or increase visceroperception in IBS patients. We investigated whether meal ingestion could increase the sensitivity of the barostat procedure for the detection of visceral hypersensitivity in IBS patients.

\section{Methods}

Seventy-one IBS patients and 30 healthy controls (HC) were included in the study. All subjects underwent a barostat procedure under fasted and postprandial conditions to measure visceroperception. Urge, discomfort, and pain were scored on a visual analog scale. Furthermore, percentages of hypersensitive IBS patients and HC were calculated and dynamic rectal compliance was assessed.

\section{Key Results}

In IBS patients, urge, discomfort, and pain scores were significantly increased postprandially vs. the fasted state. The HC showed increased scores for urge and pain only. Rectal dynamic compliance remained unaltered in both groups. Postprandial hypersensitivity percentages did not significantly differ vs. the fasted state in IBS patients, nor in HC.

\section{Conclusions \& Inferences}

Postprandial barostat measurement enhances visceroperception in IBS but has no added value to detect visceral hypersensitivity in individual IBS patients. 


\section{Introduction}

Irritable bowel syndrome (IBS) is a frequently occurring functional gastrointestinal disorder, characterized by abdominal discomfort or pain associated with changes in bowel habits ${ }^{1,2}$. In the Western world, IBS affects up to $20 \%$ of the population and is more frequent in women than men $^{1}$. One of the mechanisms involved in symptom generation and in the pathophysiology of IBS is visceral hypersensitivity ${ }^{3,4}$. In 1973, Ritchie et al. were the first to demonstrate increased pain responses to rectal balloon distensions in IBS patients compared to healthy individuals ${ }^{5}$. Since then, several studies have confirmed that perception of pain, but also of urge and discomfort in response to rectal distension, is increased in IBS compared to healthy controls $(\mathrm{HC})^{5-10}$. This is referred to as visceral hypersensitivity and is associated with symptoms ${ }^{3,11-14}$. Studies have reported different results with respect to the prevalence of visceral hypersensitivity in IBS patients, with rates varying between $33 \%$ and $90 \%^{3,9-12,15}$. In IBS, meal ingestion often provokes or aggravates symptoms ${ }^{16-20}$. In several studies, the effect of nutrients on visceral perception in IBS has been assessed ${ }^{23-29}$. As fat-rich food in particular is reported to induce abdominal complaints in $\mathrm{IBS}^{17,21,22}$, most investigators have used food with a high-fat content to modulate visceral perception ${ }^{23-28}$. These studies found that pain perception in the fasted state was increased in IBS patients vs. HC. Additionally, they showed a postprandial increase in visceral perception compared to the fasted state in IBS patients but not in controls. These observations indicate that nutrients and fat in particular, have an important role in the modulation of visceral perception in IBS. However, when analyzing these studies in more detail, it is obvious that calorie-density varied markedly among studies and also that lipids or fat were often administered intraduodenally ${ }^{23-25,28}$, which is not a physiologic route.

As food triggers IBS symptoms and visceroperception is a potential biomarker for IBS, we hypothesize that oral ingestion of an 'average size' meal will increase sensitivity of the visceroperception test in IBS patients compared to measurement in the fasted state. We also postulate that ingestion of a meal will increase hypersensitivity in IBS patients and thereby potentially enhance the discriminative capacity between IBS and HC.

\section{Materials and methods}

\section{Participants}

A total of 71 IBS patients diagnosed according to the Rome III criteria, were included via routine clinical care for barostat measurement, at the outpatient clinic of the division 
Gastroenterology-Hepatology of the Maastricht University Medical Center+. Thirty healthy volunteers were recruited via local advertisements. Before participation, a brief medical history was taken to exclude the presence of gastrointestinal disorders. Informed consent was obtained prior to their participation. The study protocol had been approved by the Ethics Committee of the Maastricht University Medical Center+ and was executed according to the Declaration of Helsinki (59th general assembly of the WMA, Seoul, South Korea, October 2008). The study has been registered in the US National Library of Medicine (http://www.clinicaltrials.gov, NCT00775060).

Sample size was determined using OpenEpi Sample Size Calculator (Emory University, Atlanta, GA, USA). The primary aim was to assess the effect of a meal on visceral perception in IBS patients. Based on data from Simrén ${ }^{23}$, it was estimated that 36 subjects would provide a power of $80 \%$ to detect an $8 \mathrm{mmHg}$ pressure change of the pain threshold before and after the meal, assuming a variance of 12 and a two-sided significance level of 0.05 . Assuming that $50 \%$ of IBS patients are hypersensitive, we aimed to include 72 patients with IBS. As secondary aim, we studied the effect of a meal in $\mathrm{HC}$ and compared these findings with those in IBS patients.

\section{Protocol}

All patients and $\mathrm{HC}$ underwent two barostat measurements interspaced with a $15 \mathrm{~min}$ break that was followed by ingestion of a standard liquid meal before the second barostat measurement. The barostat protocol for the assessment of rectal sensitivity and dynamic compliance was performed as described previously by Vanhoutvin et al. ${ }^{30}$.

\section{Meal}

The liquid meal consisted of $200 \mathrm{ml}$ of Nutridrink $^{\circledR}$ (Nutricia Nederland B.V.; Zoetermeer, the Netherlands), enriched with $15 \mathrm{ml} \mathrm{Calogen}{ }^{\circledR}$ (50 g fat per $100 \mathrm{ml}$ ) (Nutricia Nederland B.V.). Total energy density of the drink was $368 \mathrm{kcal}$, and included $12.0 \mathrm{~g}$ proteins, $36.8 \mathrm{~g}$ carbohydrates, and $19.1 \mathrm{~g}$ fat.

\section{Barostat}

Participants arrived in the hospital after an overnight fast. They self-administered a rectal enema containing $60 \mathrm{ml}$ of tap water to clean the rectum and were instructed to void rectal contents 5 min thereafter.

Rectal perception and compliance were measured with an electronic barostat (Distender II; G\&J Electronics, Toronto, ON, Canada, part: C7-CB-R). During the rectal barostat investigation, a commercially available barostat balloon of non-compliant material (Mui Scientific, Missisauga, ON, Canada, part: C7-2CB-R) was lubricated with KY-gel (Johnsson \& Joshnsson, Longhorne, PA, USA) and inserted into the rectum, $4 \mathrm{~cm}$ 
proximal to the anal sphincter. Balloon distensions were controlled using a standard software package (Version 6.7; G\&J Electronics).

The barostat protocol consisted of four subsequent procedures (Figure 2.1) for the assessment of visceral perception and rectal compliance, respectively. In both patients and controls, only subpart 3 and 4 of the barostat protocol were repeated during the second, postprandial measurement:

1. Balloon unfolding - The first part of the protocol consisted of a single distension at an absolute pressure of $25 \mathrm{mmHg}$ to sensitize the rectum and to ensure that the balloon was not leaky and placed correctly.

2. Minimal distension pressure measurement - Subsequently, to measure minimal distension pressure (MDP), a staircase distension protocol was applied with increasing pressure steps of $1 \mathrm{mmHg}$ for $30 \mathrm{~s}$ each, ranging from 0 to $20 \mathrm{mmHg}$. The minimal balloon pressure needed to overcome the intra-abdominal pressure (MDP), was detected via interference of the respiratory curves with the balloon volume curve. The obtained MDP-value was used to standardize the perception protocol (see section 3).

3. Measurement of visceral perception - A semi-random staircase protocol was applied, consisting of 17 pressure steps between 0 and $50 \mathrm{mmHg}$ above MDP, with increments of $3 \mathrm{mmHg}$. Duration of each pressure step was $1 \mathrm{~min}$, interspaced with a $30 \mathrm{~s}$ interval at MDP. Thirty seconds after initiation of each pressure step urge, discomfort, and pain were scored, using a visual analog scale (VAS) with a range between 0 and $100 \mathrm{~mm}$.

4. Compliance - After finishing the visceral perception measurement, MDP was set to zero. Rectal compliance was then measured using a staircase distension protocol with pressure steps of $5 \mathrm{mmHg}$ each (range $0-50 \mathrm{mmHg}$ ) and a duration of $30 \mathrm{~s}$. Rectal dynamic compliance was assessed by calculating the maximum volume increase between two pressure steps over the compliance protocol, divided by the pressure difference of the two steps.

\section{Visceral hypersensitivity}

Fasting and postprandial visceral hypersensitivity were calculated according to the definition used by van der Veek et al. for each time point, i.e. the mean pain threshold in $\mathrm{HC}$ minus $2 \mathrm{SD} .{ }^{15}$ Using the data of this study, the fasted mean pain threshold in $\mathrm{HC}$ was $11 \mathrm{mmHg}$ above MDP, whereas the postprandial mean pain threshold in HC was $8 \mathrm{mmHg}$ above MDP. A VAS-score $>10 \mathrm{~mm}$ for pain at or before this threshold indicated hypersensitivity. According to this cut-off, percentages IBS patients and $\mathrm{HC}$ classified as hypersensitive in the fasted and postprandial state were compared. 


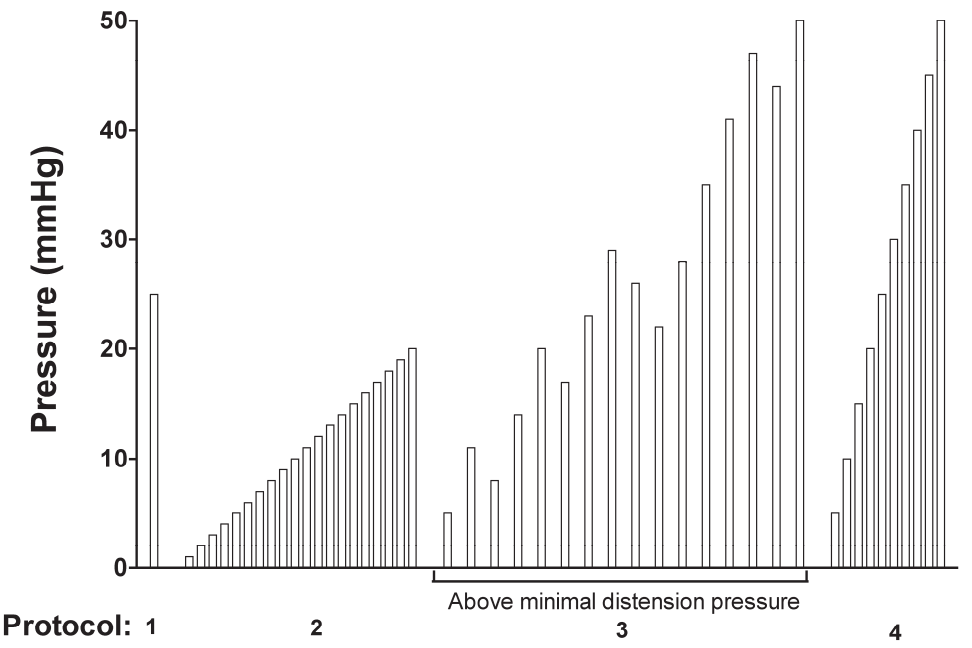

Figure 2.1

Barostat protocol with (1) sensitization; (2) measurement of MDP;

(3) assessment of visceroperception, and (4) measurement of rectal dynamic compliance.

\section{Statistical analyses}

Urge, discomfort, pain, and compliance curves were analyzed using a Gaussian nonlinear regression, including a random effect and an autocorrelation ${ }^{30}$. The effect of a meal on urge, discomfort, and pain and on rectal dynamic compliance was considered significant if the $90 \%$ confidence intervals (Cls) did not overlap ${ }^{31}$. Furthermore, differences of area under curves (AUCs) between IBS patients and HC were calculated in the fasted $v s$. the postprandial state to assess and quantitate the effect of meal ingestion between groups. A Wilcoxon signed rank test was used to compare thresholds for urge, discomfort, and pain in the fasting vs the postprandial state. Dichotomous variables were compared with a McNemar cross-tabulation within groups and using a chi-square test between groups (SPSS 16.0 for Macintosh, Chicago IL, USA). A Mann-Whitney $U$-test was used to compare age between IBS patients and controls. A two-sided $P$-value below 0.05 was considered to be statistically significant.

\section{Results}

\section{Baseline characteristics}

Mean age $( \pm$ SEM) did not differ between IBS patients and HC $(38.4 \pm 1.7$ years and $36.6 \pm 3.1$ years, respectively; $P=0.59$ ). Of the IBS patients, $70 \%$ was female compared to 
$63 \%$ in the HC. In the IBS patients, bowel habits for diarrhea (IBS-D), constipation (IBSC), and mixed stool pattern (IBS-M) were $49 \%, 31 \%$, and $20 \%$, respectively.

\section{Effect of a meal within groups}

Figures 2.2-4 show the non-linear regression curves (with their corresponding Cl's) of visceral perception, i.e., urge, discomfort, and pain, in IBS patients and HC. Cl's were very small in all cases.

\section{Urge}

According to the Cls, scores for urge were significantly higher during the postprandial measurement compared to the fasted state in IBS patients. The increase was observed within the pressure range $5-50 \mathrm{mmHg}$. The $\mathrm{HC}$ also showed a significant increase of urge, over the whole pressure range of the protocol in the postprandial vs. the fasted state, which is between 0 and $50 \mathrm{mmHg}$ (Figure 2.2).

IBS patients

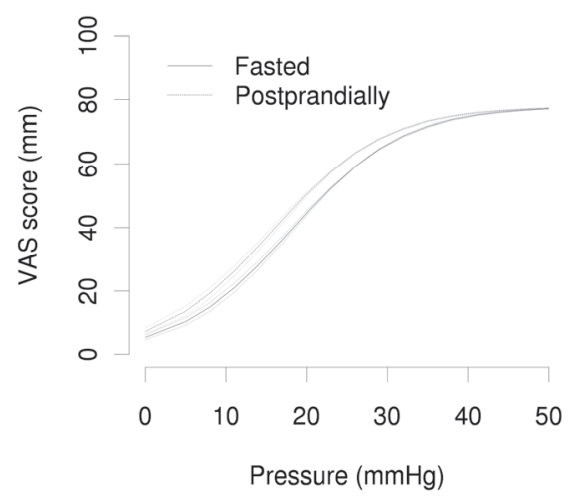

Healthy controls

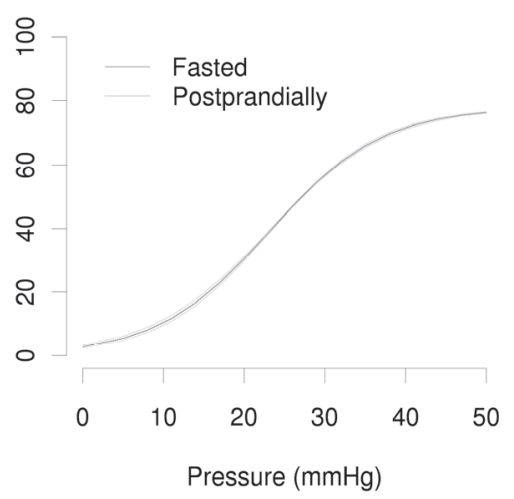

Figure 2.2 Urge scores in IBS patients and healthy controls under fasted and postprandial conditions.

\section{Discomfort}

Scores for discomfort were significantly increased in IBS patients over the pressure range $(5-47 \mathrm{mmHg})$, during the postprandial measurement vs. fasting. Discomfort scores in HC did not differ significantly between the fasted vs. postprandial measurements (Figure 2.3). 
IBS patients

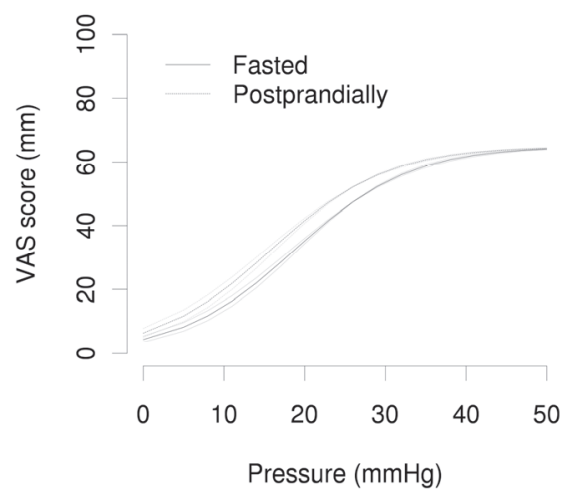

Healthy controls

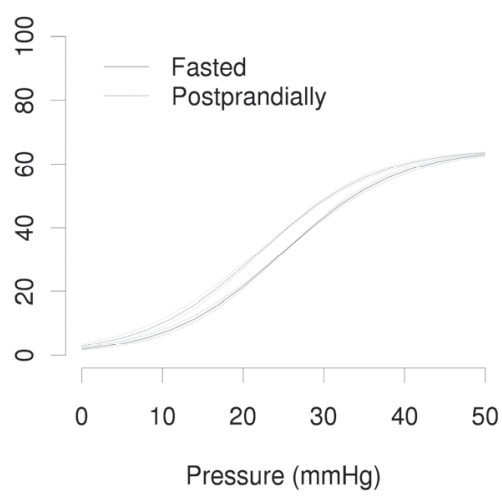

Figure 2.3 Discomfort scores in IBS patients and healthy controls under fasted and postprandial conditions.

\section{Pain}

Within IBS patients, as well as in HC, postprandial scores for pain were significantly increased over the whole pressure range, compared to the fasted state (Figure 2.4).

IBS patients

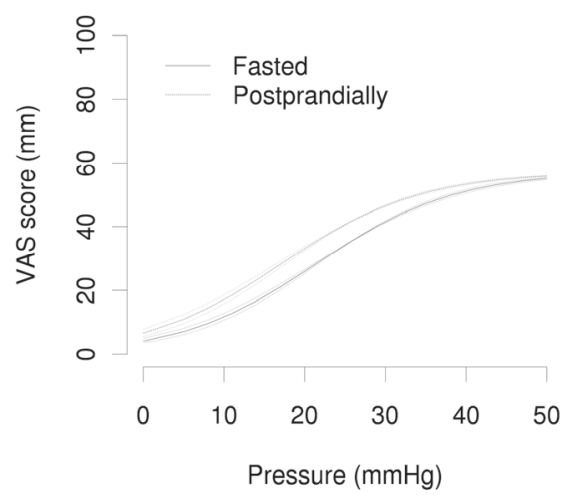

Healthy controls

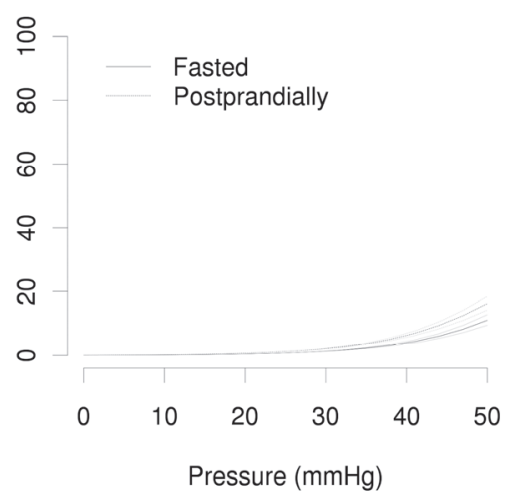

Figure 2.4 Pain scores in IBS patients and healthy controls under fasted and postprandial conditions. 


\section{Thresholds}

In Figure 2.5, the thresholds for urge, discomfort, and pain are shown. A significant increase of postprandial vs. fasting median thresholds was found for urge [20

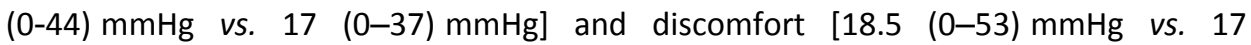
$(0-53) \mathrm{mmHg}$ ] in $\mathrm{HC}$ and for discomfort in IBS patients [14 (0-50) $\mathrm{mmHg}$ vs. 11 $(0-50) \mathrm{mmHg}$. A significant postprandial decrease was found for pain in IBS patients [14 (0-53) mmHg vs. $17(0-53) \mathrm{mmHg}$, while no differences were found for pain in $\mathrm{HC}$, nor for urge in IBS patients.
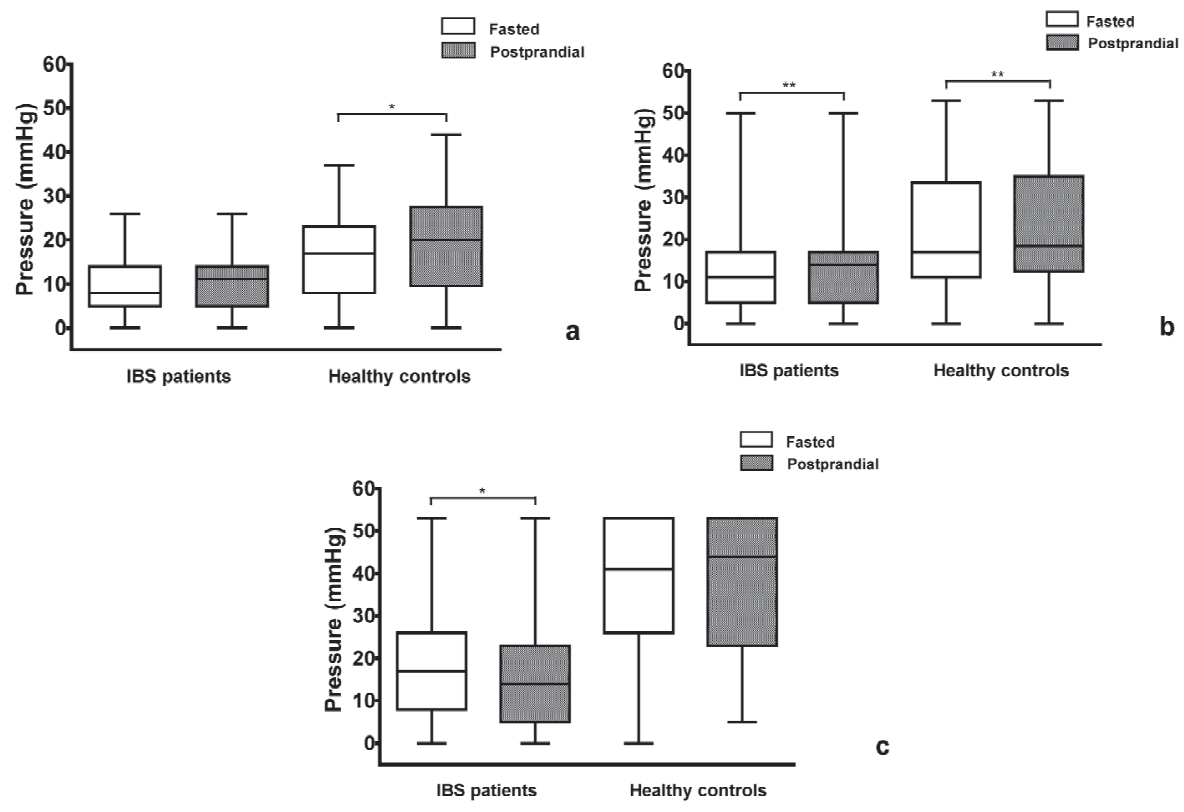

Figure 2.5 A-C Thresholds for urge (A), discomfort (B), and pain (C) in IBS patients and healthy controls, under fasted vs postprandial conditions. ${ }^{*} P<0.05 ;{ }^{*} P<0.01$.

\section{Visceral hypersensitivity}

Figure 2.6 shows that the percentages of IBS patients and HC with hypersensitivity in the fasted vs. postprandial state were not significantly different: for IBS patients $40.9 \%$ (29 of 71 ) vs. $39.4 \%$ (28 of 71 ) and for HC 6.7\% (2 of 30) vs. 3.3\% (1 of 30), based on previously defined criteria. ${ }^{15}$ Postprandially, four normosensitive IBS patients became hypersensitive, whereas five hypersensitive patients became normosensitive. With respect to IBS subgroups, no significant changes were observed in the percentage 
patients with hypersensitivity before compared to after meal intake: $34.3 \%$ vs $40.0 \%$ in IBS-D ( $P=0.73) ; 45.5 \%$ vs. $31.8 \%$ in IBS-C $(P=0.38)$ and $50.0 \%$ vs. $50.0 \%$ in IBS-M ( $P=1.00)$.

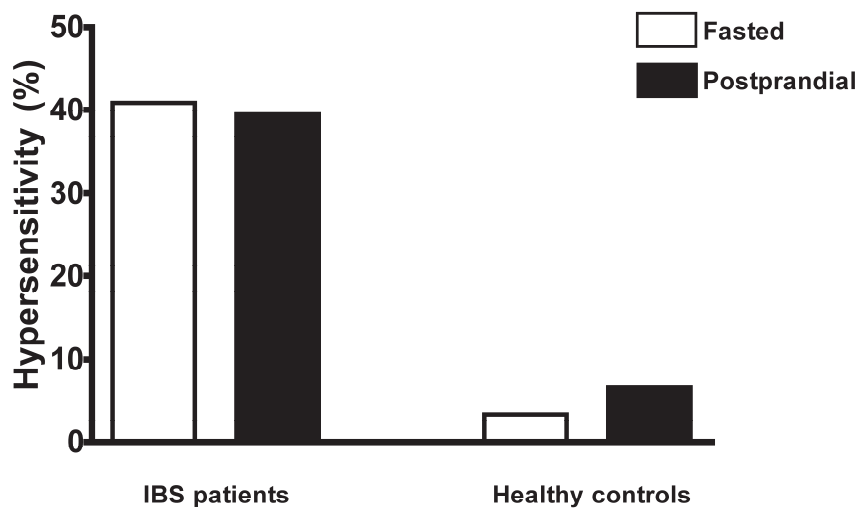

Figure 2.6 Fasted and postprandial hypersensitivity percentages in IBS patients and healthy controls.

\section{Rectal dynamic compliance}

As shown in Figure 2.7, rectal dynamic compliance remained unaltered in the postprandial state, compared to the fasted state in both groups.

IBS patients

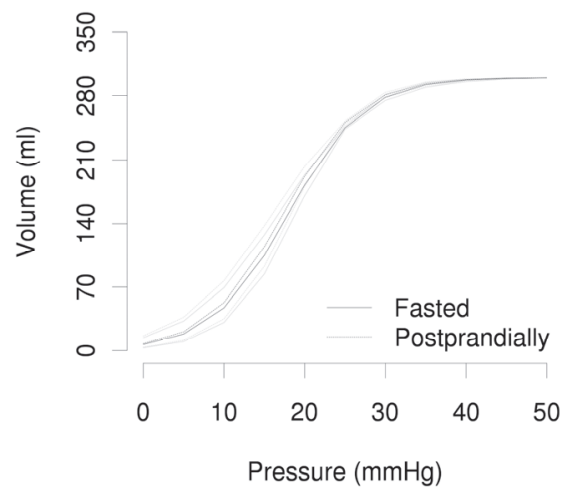

Healthy controls

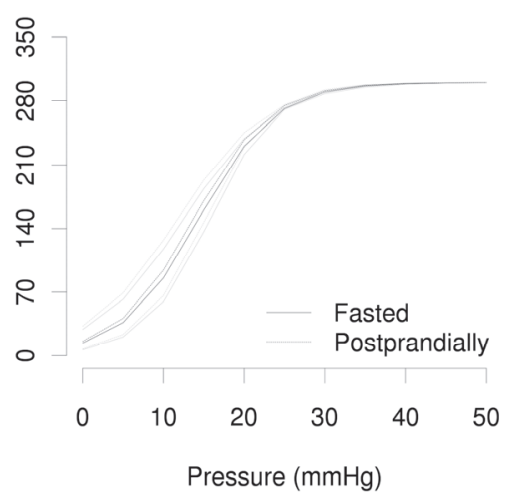

Figure 2.7 Rectal dynamic compliance in IBS patients and healthy controls under fasted and postprandial conditions. 


\section{Effect of meal between groups}

\section{Urge, discomfort, pain, and rectal dynamic compliance}

The difference in perception scores for urge and pain between IBS patients and HC was significantly larger in the postprandial state compared to the fasted state. No such difference was found for discomfort (Table 2.1). Furthermore, according to the confidence intervals, no differences between IBS patients and $\mathrm{HC}$ were found for rectal dynamic compliance in the fasted, nor in the postprandial state.

Table 2.1 Differences of visceral perception between IBS patients and HC in the fasted vs. the postprandial state for urge, discomfort, and pain

\begin{tabular}{ll}
\hline Perception parameter & Delta AUC of IBS patients $-\mathrm{HC}[90 \% \mathrm{CI}]$ \\
\hline Urge* & $367.13[307.89-426.84]$ \\
$\quad$ Fasted & $535.97[475.01-597.50]$ \\
Postprandial & \\
Discomfort & $377.99[306.64-450.48]$ \\
$\quad$ Fasted & $371.91[296.49-488.06]$ \\
$\quad$ Postprandial & \\
Pain* & $1497.02[1452.90-1540.61]$ \\
Fasted & $1655.57[1604.922-1705.75]$ \\
Postprandial & \\
\hline
\end{tabular}

The asterisks indicate significant differences between the deltas of the area under curve (AUC) of irritable bowel syndrome (IBS) patients minus healthy controls $(\mathrm{HC})$ in the fasted vs the postprandial state.

\section{Discussion}

The primary aim of this study was to investigate whether a liquid meal increased the sensitivity of the barostat procedure. We found an increased visceral perception in IBS patients for urge, discomfort, and pain after meal ingestion, and for urge and pain in HC. Despite the increase in visceroperception postprandially, the overall percentage of IBS patients with visceral hypersensitivity did not increase. Rectal compliance remained unaltered after meal intake.

Findings from this study confirm previous literature data with respect to an increase in visceral sensitivity in IBS patients vs healthy subjects ${ }^{5-10}$. Several mechanisms have been postulated to be responsible for this altered perception, mainly aiming at increased perception and processing of pain by IBS patients ${ }^{32-34}$. In addition, there is growing evidence suggesting that peripheral immune mechanisms and disturbed neuro-immune communication may play a role in the hypersensitivity observed in IBS patients $b^{36}$.

Our data confirm that a high-fat meal may augment visceral sensory responses of IBS patients as was previously shown after intraduodenal fat administration ${ }^{23-25,28}$. Only 
two other studies have assessed the effect of meal intake on visceroperception via the physiologic route, i.e. after oral ingestion ${ }^{26,27}$. Van der Veek et al. assessed the effect of a fat-rich liquid meal with a caloric load of $600 \mathrm{kcal}$ [39 energy (EN)\% fat, i.e., $234 \mathrm{kcal}$ ), on visceral perception in 26 IBS patients and $13 \mathrm{HC}$. They found that postprandial but not fasting pain scores were significantly elevated in IBS patients compared to $\mathrm{HC}^{27}$. Simrén et al. studied 11 IBS patients and 13 healthy subjects in the fasted state and after a meal with a high caloric load (i.e., $800 \mathrm{kcal}$ ) and low in fat (20 EN\% fat, i.e. $160 \mathrm{kcal}$ ) or high in fat (60 EN\% fat, i.e. $480 \mathrm{kcal}$ ). In IBS patients only, rectal sensitivity increased significantly (i.e. lower thresholds for discomfort and pain and higher perceived pain intensity by VAS) at 30 and 60 min postprandially after the high-fat meal, but not after the low-fat meal.

These studies suggest that the fat load per se is a stronger stimulus than the caloric load, which justifies the composition of the meal used in this study, with a caloric load comparable to an average meal but high in fat. Whereas the numbers of patients and controls were relatively small in both above mentioned studies, this study included a higher number of patients, representing a non-selected general IBS population. In addition, measurements were performed as part of routine clinical care, which allowed us to show that increased meal-induced visceroperception is present in the general IBS population.

Although the IBS subtypes did not show any significant differences in their reaction to the liquid meal with regards to hypersensitivity, this remains inconclusive as this study was not powered to show differences between IBS subtypes.

We cannot exclude that the order of the repeated barostat measurements has affected viscerosensation, explaining the increased postprandial visceroperception observed in both the IBS patients and HC. However, previous studies that assessed the effect of a meal on postprandial perception, either used a similar design ${ }^{24,25,27}$ or performed measurements on separate days ${ }^{23,26}$, and even used a conditioning distension ${ }^{26}$ to account for repeated measurements ${ }^{35}$. Despite methodological issues, in all these studies it was found that visceral perception increased postprandially ${ }^{23-28}$, meaning that the order of measurements has little effect on meal-induced increased visceroperception.

At group level, both fasted and postprandial perception scores to rectal distensions were increased in IBS patients, compared to HC. The shifts that we observed in the pain threshold of IBS patients are in line with the above mentioned observations, although this could not be found for urge and discomfort. Nonetheless, one has to keep in mind that the data from the thresholds are based on a single pressure level, whereas the perception curves include the full pressure range between 0 and $50 \mathrm{mmHg}$.

Although we demonstrated that postprandial perception of urge, discomfort, and pain increased in IBS patients, no postprandial effect could be detected on the percentage of IBS patients that were classified as hypersensitive. In total, $86 \%$ of IBS patients being 
hypersensitive in the postprandial state were also hypersensitive under fasting conditions. The postprandial increase in visceroperception as indicated by the perception curves is therefore likely to the result of an increased perception of patients that were already hypersensitive. The net effect is an overall increase of visceral perception of urge, discomfort, and pain on a group level, but not on the presence of visceral hypersensitivity as assessed on an individual level.

With respect to characteristics of the nine patients in whom the 'sensitivity status' changed between the fasting and postprandial state, eight were females. The age of the five hypersensitive patients who became normosensitive appeared to be lower compared to the four normosensitive patients who became hypersensitive (29.8 vs. 46.3 years). In more detail, under fasting conditions, six of the nine patients had VASscores just above or below the threshold level. Therefore, a minor shift in the VAS did result in the change in their sensitivity status.

Oral administration of a meal increases overall visceral perception, and decreases rectal compliance in IBS patients compared to HC. However, the sensitivity of the barostat procedure is not increased as the discriminative capacity between IBS patients and HC based on visceral hypersensitivity remained unaltered. It is important to note that definitions for hypersensitivity differ between studies. The present findings implicate that a well-defined measure for visceral hypersensitivity is warranted, especially for clinical applications.

A possible limitation of our study included the short 15 min recovery time between two measurements. Data from the studies by Simrén et al. and van der Veek et al. provided substantial evidence that even a short recovery time of 5-15 $\min$ was found to be sufficient to show an effect of meal ingestion on visceroperception ${ }^{26,27}$. Furthermore, this recovery time limited the burden for patients and $\mathrm{HC}$ by combining the fasted and postprandial barostat measurements with a fixed order of experiments (i.e. first fasted, then postprandial). This allowed us to test a large group of patients.

Currently, barostat examinations are not used ubiquitously as routine procedures in the work-up of patients with IBS to determine visceroperception. Visceral hypersensitivity, however, is considered a biomarker for at least a substantial subgroup of IBS patients but the postprandial condition does not facilitate higher discrimination between IBS patients and $\mathrm{HC}$.

In conclusion, the results obtained by this study indicate that ingestion of a liquid meal modulates visceral perception in IBS. Meal ingestion prior to a barostat measurement induces different responses in IBS patients compared to healthy subjects. However, it does not increase the diagnostic yield of the barostat measurement to detect visceral hypersensitivity in the individual IBS patient. The majority of the IBS patients with postprandial hypersensitivity have shown to be already hypersensitive under fasting conditions. 
When searching for biomarkers for IBS and diagnostic tools to detect altered visceroperception in IBS in clinical practice, it is important to be able to detect hypersensitivity by a short lasting, standardized, accurate, and preferably physiologic test procedure. Future research should focus on optimizing a test for the assessment of visceral hypersensitivity. 


\section{References}

1. Thompson WG, Longstreth GF, Drossman DA, Heaton KW, Irvine EJ, Muller-Lissner SA. Functional bowel disorders and functional abdominal pain. Gut. 1999;45:II43-7.

2. Drossman DA, Camilleri M, Mayer EA, Whitehead WE. AGA technical review on irritable bowel syndrome. Gastroenterology. 2002;123:2108-31.

3. Mertz H, Naliboff B, Munakata J, Niazi N, Mayer EA. Altered rectal perception is a biological marker of patients with irritable bowel syndrome. Gastroenterology. 1995;109:40-52.

4. Prior A, Sorial E, Sun WM, Read NW. Irritable bowel syndrome: differences between patients who show rectal sensitivity and those who do not. Eur J Gastroenterol Hepatol. 1993;5:343-9.

5. Ritchie J. Pain from distension of the pelvic colon by inflating a balloon in the irritable colon syndrome. Gut. 1973; 4:125-32.

6. Whitehead WE, Holtkotter B, Enck P, Hoelzl R, Holmes KD, Anthony J, Shabsin HS, Schuster MM. Tolerance for rectosigmoid distension in irritable bowel syndrome. Gastroenterology. 1990;98: 1187-92.

7. Munakata J, Naliboff B, Harraf F, Kodner A, Lembo T, Chang L, Silverman DH, Mayer EA. Repetitive sigmoid stimulation induces rectal hyperalgesia in patients with irritable bowel syndrome. Gastroenterology. 1997;112:55-63.

8. Naliboff BD, Munakata J, Fullerton S, Gracely RH, Kodner A, Harraf F, Mayer EA. Evidence for two distinct perceptual alterations in irritable bowel syndrome. Gut. 1997;41:505-12.

9. Bouin $M$, Meunier $P$, Riberdy-Poitras $P$. Pain hypersensitivity in patients with functional gastrointestinal disorders: a gastrointestinal-specific defect or a general systemic condition? Dig Dis Sci. 2001;46: 2542-8.

10. Bouin $M$, Plourde $V$, Boivin $M$, Riberdy $M$, Lupien $F$, Laganière $M$, Verrier $P$, Poitras $P$. Rectal distention testing in patients with irritable bowel syndrome: sensitivity, specificity and predictive values of pain sensory thresholds. Gastroenterology. 2002;122: 1771-7.

11. Posserud I, Syrous A, Lindström L, Tack J, Abrahamsson H, Simrén M. Altered rectal perception in irritable bowel syndrome is associated with symptom severity. Gastroenterology. 2007;133:1113-23.

12. Kanazawa M, Palsson OS, Thiwan SI, Turner MJ, van Tilburg MA, Gangarosa LM, Chitkara DK, Fukudo S, Drossman DA, Whitehead WE. Contributions of pain sensitivity and colonic motility to IBS symptom severity and predominant bowel habits. Am J Gastroenterol. 2008;103:2550-61.

13. Schmulson M, Chang L, Naliboff B, Lee OY, Mayer EA. Correlation of symptom criteria with perception thresholds during rectosigmoid distension in irritable bowel syndrome patients. Am J Gastroenterol. 2000;95:152-6.

14. Lee KJ, Kim JH, Cho SW. Relationship of underlying abnormalities in rectal sensitivity and compliance to distension with symptoms in irritable bowel syndrome. Digestion. 2006;73:133-41.

15. Veek van der PJ, Rood van YR, Masclee AAM. Symptom severity but not psychology predicts visceral hypersensitivity in irritable bowel syndrome. Clin Gastroenterol Hepatol. 2008;6:321-8.

16. King TS, Elia M, Hunter JO. Abnormal colonic fermentation in irritable bowel syndrome. Lancet. 1998; 352:1187-9.

17. Simrén M, Månsson A, Langkilde AM, Svedlund J, Abrahamsson H, Bengtsson U, Björnsson ES. Foodrelated gastrointestinal symptoms in the irritable bowel syndrome. Digestion. 2001;63:108-15.

18. Ragnarsson G, Bodemar G. Pain is temporally related to eating but not to defaecation in the irritable bowel syndrome (IBS). Patients' description of diarrhea, constipation and symptom variation during a prospective 6-week study. Eur J Gastroenterol Hepatol. 1998;10:415-21.

19. Lea R, Whorwell PJ. The role of food intolerance in irritable bowel syndrome. Gastroenterol Clin North Am. 2005;34:247-55.

20. Camilleri M, McKinzie S, Busciglio I, Low PA, Sweetser S, Burton D, Baxter K, Ryks M, Zinsmeister AR. Prospective study of motor, sensory, psychologic, and autonomic functions in patients with irritable bowel syndrome. Clin Gastroenterol Hepatol. 2008;6: 772-81.

21. Salvioli B, Serra J, Azpiroz F, Malgelada JR. Impaired small bowel gas propulsion in patients with bloating during intestinal lipid infusion. Am J Gastroenterol. 2006;101:1853-7. 
22. Morcos A, Dinan T, Quigley EM. Irritable bowel syndrome: role of food in pathogenesis and management. J Dig Dis. 2009;10:237-46.

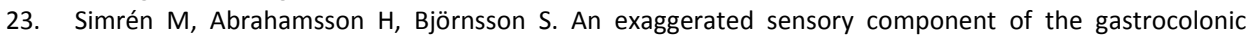
response in patients with irritable bowel syndrome. Gut. 2001;48:20-7.

24. Caldarella MP, Milano A, Laterza F, Sacco F, Balatsinou C, Lapenna D, Pierdomenico SD, Cuccurullo F, Neri M. Visceral sensitivity and symptoms in patients with constipation- or diarrhea-predominant irritable bowel syndrome (IBS): effect of a low-fat intraduodenal infusion. Am J Gastroenterol. 2005;100:383-9.

25. Simrén $M$, Abrahamsson $H$, Björnsson S. Lipid-induced colonic hypersensitivity in the irritable bowel syndrome: the role of bowel habit, sex and psychologic factors. Clin Gastroenterol Hepatol. 2007;5: 201-8.

26. Simrén $M$, Agerforz $P$, Björnsson $S$, Abrahamsson H. Nutrient-dependent enhancement of rectal sensitivity in irritable bowel syndrome (IBS). Neurogastrenterol Motil. 2007;19:20-9.

27. Veek van der PPJ, Steenvoorden J, Steens PJ, Schaar van der J, Brussee J, Masclee AAM. Recto-colonic reflex is impaired in patients with irritable bowel syndrome. Neurogastroenterol Motil. 2007;19:653-9.

28. Distrutti E, Hauer SK, Fiorucci S, Pensi MO, Morelli A. Intraduodenal lipids increase perception of rectal distension in IBS patients. Gastroenterology. 2000;118(Suppl. 2):A138.

29. Tillander J, Ringström G, Björnsson E, Abrahamsson H, Simrén M. Do duodenal protein or carbohydrate affect colonic sensorimotor function in irritable bowel syndrome (IBS) and health? Gastroenterology. 2002;122(Suppl. 1):T1333.

30. Vanhoutvin SA, Troost FJ, Kilkens TO, Lindsey PJ, Hamer HM, Jonkers DM, Venema K, Brummer RJ. Effects of butyrate enemas on visceral perception in healthy volunteers. Neurogastroenterol Motil. 2009;21:952-e76.

31. Lindsey PJ, Kaufman J. Analysis of a longitudinal ordinal response clinical trial using dynamic models. J R Stat Soc Ser C Appl Stat. 2004;53:523-37.

32. Camilleri M, McKinzie S, Busciglio I, Low PA, Sweetser S, Burton D, Baxter K, Ryks M, Zinsmeister AR. Prospective study of motor, sensory, psychologic, and autonomic functions in patients with irritable bowel syndrome. Clin Gastroenterol Hepatol. 2008;6: 772-81.

33. Mayer EA, Gebhart GF. Basic and clinical aspects of visceral hyperalgesia. Gastroenterology. 1994;107: 271-93.

34. Piché $M$, Arsenault $M$, Poitras $P$, Rainville $P$, Bouin $M$. Widespread hypersensitivity is related to altered pain inhibition processes in irritable bowel syndrome. Pain. 2010;148:49-58.

35. Hammer HF, Phillips SF, Camilleri M, Hanson RB. Rectal tone, distendability, and perception: reprocibility and response to different distensions. Am J Physiol. 1998;274:G584-90.

36. Elsenbruch S. Abdominal pain in irritable bowel syndrome: A review of putative psychological, neural and neuro-immune mechanisms. Brain Behav Immun. 2011;25:386-94. 


\section{Chapter}

Rectal hypersensitivity as hallmark for irritable bowel syndrome: defining the optimal cut-off

S Ludidi, JM Conchillo, D Keszthelyi, M van Avesaat, JW Kruimel, DM Jonkers, AAM Masclee

Neurogastroenterol Motil. 2012;24:729-733, e345-346. 


\section{Abstract}

Introduction

Visceral hypersensitivity is a frequently observed hallmark of irritable bowel syndrome (IBS). Studies have reported differently about the presence of visceral hypersensitivity in IBS resulting from lack of standardization of the barostat procedure and due to different criteria used to assess hypersensitivitiy. We aimed to calculate the optimal cut-off to detect visceral hypersensitivity in IBS.

\section{Methods}

Hundred twenty-six IBS patients and 30 healthy controls $(\mathrm{HC})$ were included for assessment of visceroperception by barostat. Pain perception was assessed on a visual analogue scale (VAS). ROC-curves were used to calculate optimal discriminative cut-off (pressure and VAS-score) between IBS patients and $\mathrm{HC}$ to define hypersensitivity. Furthermore, pain perception to distension sequences below the pressure threshold for hypersensitivity was defined as allodynia.

\section{Results}

IBS patients showed increased visceroperception compared to HC. Thresholds for first sensation and first pain were lower in IBS patients vs. HC $(P<0.01)$. ROC-curves showed optimal discrimination between IBS patients and HC at $26 \mathrm{mmHg}$ with a VAS cut-off $\geq 20 \mathrm{~mm}$. Using this criterion, hypersensitivity percentages were $63.5 \%$ and $6.6 \%$ in IBS patients and $\mathrm{HC}$, respectively. No significant differences were observed between IBS subtypes. Allodynia was found in a small number of patients (11\%).

\section{Conclusion}

Optimal cut-off for visceral hypersensitivity was found at pressure $26 \mathrm{mmHg}$ with a VAS $\geq 20 \mathrm{~mm}$, resulting in $63.5 \%$ of IBS patients being hypersensitive and $11 \%$ being allodynic. Standardization of barostat procedures and defining optimal cut-off values for hypersensitivity is warrented when employing rectal barostat measurements for research or clinical purposes. 


\section{Introduction}

Irritable bowel syndrome (IBS) is a functional disorder of the gastrointestinal (GI) tract $^{1,2}$ that is highly prevalent in the Western world ${ }^{1}$. A frequently observed feature of IBS is visceral hypersensitivity, which refers to increased perception of physiological and non-physiological chemical or mechanical stimuli applied to the GI tract $^{3-7}$. Several studies have shown that visceral hypersensitivity to mechanical distension of the gut in IBS is related to symptom severity ${ }^{8-10}$ and it is considered to play a role in the pathophysiology of IBS ${ }^{8-12}$.

Visceral hypersensitivity consists of two components, i.e. allodynia and hyperalgesia. Allodynia is defined as the perception of pain following non-painful or non-noxious stimuli, while hyperalgesia refers to an increased pain perception following painful or noxious stimuli ${ }^{4}$. Although the exact mechanistic role of hypersensitivity and its separate components in IBS is not yet known, it has been postulated as a biological marker in IBS patients ${ }^{13}$.

Interventions or therapies targeting on the enteric nervous system, may be able to modify visceroperception in IBS patients ${ }^{14}$. Meals for example, are known to provoke or aggravate symptoms in $\mathrm{IBS}^{15-18}$. Nonetheless, we have recently shown that measuring visceral hypersensitivity in the postprandial state does not result in better differentiation between IBS patients and controls ${ }^{19}$. Modulation of the enteric nervous system by the serotonin receptor-3 antagonist alosetron however, has previously shown to increase thresholds to distension in IBS patients with diarrhoea ${ }^{20}$. Other routes or modulating factors that may be involved in the aetiology of altered viscerosensation in IBS, are corticotropin releasing hormone, nitric oxide and serine proteases $^{14,21-23}$. Assessment of visceral hypersensitivity may be helpful to diagnose IBS, to identify IBS subgroups that share the same pathophysiological mechanism and to select IBS patients for therapeutic clinical trials or tailored treatment $t^{8,10-13,24,25}$. Furthermore, distinguishing allodynia as being a part of hypersensitivity may guide identification of potential mechanistic factors involved in IBS.

In GI motility units, a rectal barostat test is the procedure employed to measure visceral sensitivity. Several studies have shown that, compared to healthy controls (HC), $33 \%$ to $90 \%$ of an IBS population is hypersensitive to rectal distensions ${ }^{8,10-12,26}$. Several factors may contribute to the observed wide range of hypersensitity among different IBS populations. First, differences may exist in patient characteristics between various IBS populations. Second, barostat protocols and paradigms used to assess visceroperception differ among research groups. Third, differences exist in criteria used to define visceral hypersensitivity. Not only perception curves have been used to 
discriminate between different groups ${ }^{27}$, but also pressure thresholds $(\mathrm{mmHg})$ for visceroperception $^{12,13,28-30}$ as well as VAS cut-off values.

In addition to standardization of barostat protocols, defining the optimal cut-off parameter and cut-off value is crucial for detection of hypersensitivity in an individual patient. Therefore, the present study was undertaken to calculate the optimal cut-off to detect rectal hypersensitivity in a prospective cohort of IBS patients versus healthy controls. Special attention was paid to IBS subtypes and the presence of allodynia.

\section{Methods}

Subjects with IBS seen at our outpatient department between January 2008 and April 2010 were asked to participate in this study. Upon approval they underwent a rectal barostat procedure at the motility research unit at the Maastricht University Medical Center+.

\section{Participants}

In that time span barostat data were available of 126 IBS patients diagnosed according to the Rome III criteria. Patients were recruited via the outpatient department of the division of Gastroenterology-Hepatology of the Maastricht University Medical Centert. Medical history was taken by a gastroenterologist and sigmoidoscopy or colonoscopy, abdominal imaging by ultrasonography or CT scan were performed to exclude organic disease $^{31}$. Thirty healthy subjects were included as controls. Before participation, a brief medical history was taken to exclude the presence of gastrointestinal disorders. Seventy-one out of 126 IBS patients and all healthy subjects had participated in a previous study on the effect of meal ingestion on visceroperception, recently published by our group ${ }^{19}$.

All subjects had signed an informed consent prior to their barostat measurement, in which they agreed upon using their data for further scientific analyses. The study protocol had been approved by the Maastricht University Medical Centre+ Committee of Ethics and was executed according to the Declaration of Helsinki $\left(59^{\text {th }}\right.$ general assembly of the WMA, Seoul, South Korea, Oct. 2008). The study has been registered in the US National Library of Medicine (http://www.clinicaltrials.gov, NCT00775060).

\section{Protocol}

All patients and $\mathrm{HC}$ underwent a rectal barostat measurement for the assessment of visceral sensitivity, as described by Vanhoutvin et al. $^{27}$. The barostat measurement was 
performed under fasting conditions and consisted of four subsequent procedures, which are briefly described below.

\section{Barostat}

Participants arrived in the hospital after an overnight fast. They self-administered a rectal enema containing $60 \mathrm{ml}$ of tap water to clean the rectum and were instructed to void rectal contents five minutes thereafter.

Rectal perception and compliance were measured with an electronic barostat (Distender II; G\&J Electronics, Toronto, ON, Canada, part: C7-CB-R). During the rectal barostat investigation, a commercially available barostat balloon of non-compliant material (Mui Scientific, Missisauga, ON, Canada, part: C7-2CB-R) was lubricated with KY-gel (Johnsson \& Joshnsson, Longhorne, PA, USA) and inserted into the rectum, $4 \mathrm{~cm}$ proximal to the anal sphincter. Balloon distensions were controlled using a standard software package (Protocol Plus Deluxe, version 6.7; G\&J Electronics).

The barostat protocol consisted of three subsequent procedures for the assessment of visceral perception. The first part of the protocol consisted of a single distension at $25 \mathrm{mmHg}$ to sensitise the rectum and to ensure that the balloon was not leaky and placed correctly. Subsequently, to measure minimal distension pressure (MDP), a staircase distension protocol was applied with increasing pressure steps of $1 \mathrm{mmHg}$ for 30 seconds each, ranging from $0-20 \mathrm{mmHg}$. The minimal balloon pressure needed to overcome the intra-abdominal pressure (MDP), was detected via interference of the respiratory curves with the balloon volume curve. The obtained MDP-value was used to standardise the perception protocol (see section 3). Finally, a semi-random staircase protocol was applied, consisting of 17 pressure steps between 0-50 $\mathrm{mmHg}$ above MDP. Duration of each pressure step was 1 minute, interspaced with a 30 second interval at MDP. Thirty seconds after initiation of each pressure step urge, discomfort and pain were scored, using a visual analogue scale (VAS) with a range between 0-100 mm. For subjects that did not complete the full protocol, VAS-scores for the remaining pressure steps were replenished, using the highest VAS-score achieved over the protocol thus far.

\section{Visceral sensitivity}

Perception data of IBS patients and HC were compared for overal pain perception, first sensation and first pain. Subsequently, visceral hypersensitivity was calculated based on pain perception according to three different criteria: 
1. Mean threshold for first pain sensation assessed in $\mathrm{HC}$ (i.e. pressure at which a VAS-score for pain > $10 \mathrm{~mm}$ was achieved for the first time) minus 2SD, as previously defined by van der Veek et al. ${ }^{8}$

2. Tenth percentile of the threshold for first pain sensation in $\mathrm{HC}$

3. Discriminative optimum (VAS and pressure) between IBS patients and HC, obtained via receiver operating characteristic (ROC) curves of pain perception (see: statistical analyses)

\section{Allodynia}

The percentage of subjects with allodynia, refering to pain perception following nonpainful or non-noxious stimuli ${ }^{4}$, were calculated for IBS patients and HC. The cut-off for first pain sensation (VAS>10 mm; see next paragraph) was used to calculate allodynia. Hence, a VAS-score $>10 \mathrm{~mm}$ prior to the pressure step of the discriminative optimum was set to indicate allodynia.

\section{First pain and first sensation}

The threshold for first pain was defined as a VAS-score $>10 \mathrm{~mm}$ for the parameter pain. Urge, discomfort and pain were measured to assess first sensation. First sensation was defined as the pressure threshold of the first of these parameters exceeding $10 \mathrm{~mm}$ at the VAS. If first pain or first sensation was not achieved during the consecutive steps of the protocol (ranging from $0-50 \mathrm{mmHg}$ ), the first following (fictive) pressure, i.e. $53 \mathrm{mmHg}$, was used to indicate first sensation.

\section{Statistical analyses}

For each pressure step, VAS cut-off values for hypersensitivity were set between 10-30 mm, with increments of $5 \mathrm{~mm}$. Subsequently, sensitivity (SEN), specificity (SPE), positive predictive value (PPV) and positive likelihood ratio ( $(R+)$ were calculated for all combinations. Using these parameters, the optimal discriminative cut-off for visceral hypersensitivity In IBS versus HC was selected, based on the highest area under ROC curve (AUC) and LR+.

A non-parametrical Mann-Whitney $U$ test was used to compare age, thresholds of first sensation and first pain. A Chi-square test with Fisher exact, when necessary, was used to compare dichotomous variables between groups. Data was analysed using Predictive Analytics SoftWare Statistics 18.0, (PASW Statistics for Macintosh, Chicago IL). A twosided $P$-value below 0.05 was considered to be statistically significant. 


\section{Results}

\section{Baseline characteristics}

Mean age $( \pm$ SEM) was comparable for IBS patients and $\mathrm{HC}(38.4 \pm 1.3$ versus $36.6 \pm 3.1$ years, respectively). Of the IBS patients $66 \%$ was female versus $63 \%$ in the $\mathrm{HC}$. Predominant bowel habits for diarrhoea (IBS-D), constipation (IBS-C) and mixed stool pattern (IBS-M), as well as unspecified stool pattern (IBS-U) were $50.0 \%, 30.0 \%, 6.5 \%$ and $13.5 \%$, respectively, in IBS patients.

\section{Visceral perception of IBS patients and HC}

Compared to HC, pain perception in IBS patients was significantly increased over the whole range of the perception protocol (Figure 3.1), as indicated by the areas under the curves (1821 [1556-2087] vs. 506 [181-832]; arbitrary units for IBS vs. HC, respectively).

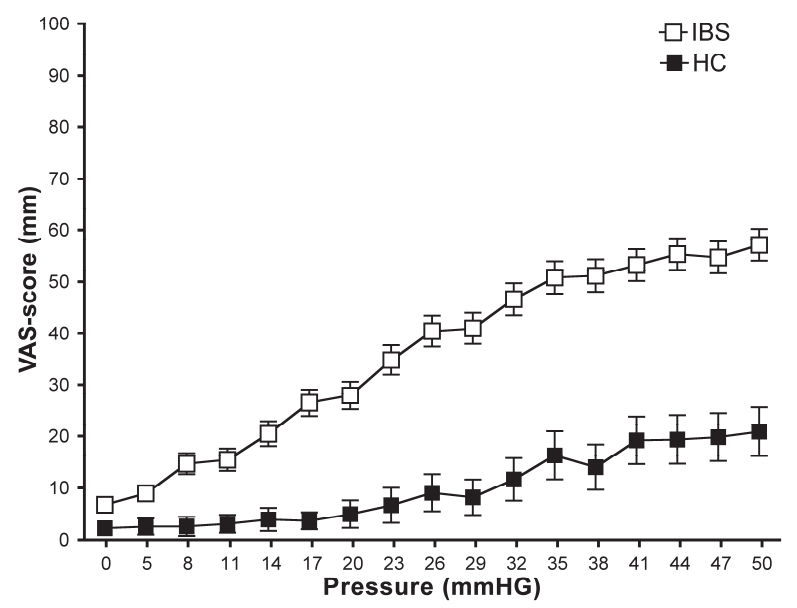

Figure 3.1 Pain perception (VAS) of IBS patients (open squares) and HC (black squares) following rectal distensions between 0 and $50 \mathrm{mmHg}$ above MDP.

\section{Distension thresholds: first sensation and first pain}

As shown in Figure 3.2a and 3.2b, median threshold for first sensation was significantly decreased in IBS patients when compared to $\mathrm{HC}(8$ [0-53] vs. 17 [0-37] $\mathrm{mmHg}$, respectively, $P<0.01)$. The threshold for first pain was also significantly reduced in IBS patients versus $\mathrm{HC}(17[0-53]$ vs. $41[0-53] \mathrm{mmHg} ; P<0.01)$. 
a

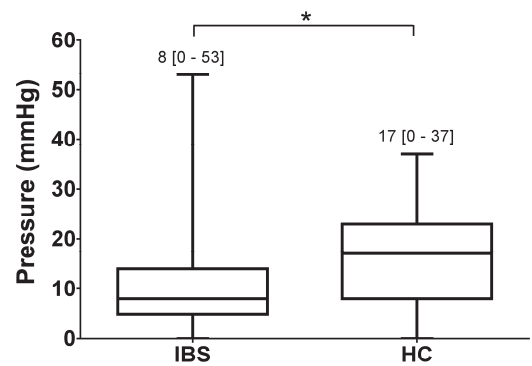

b

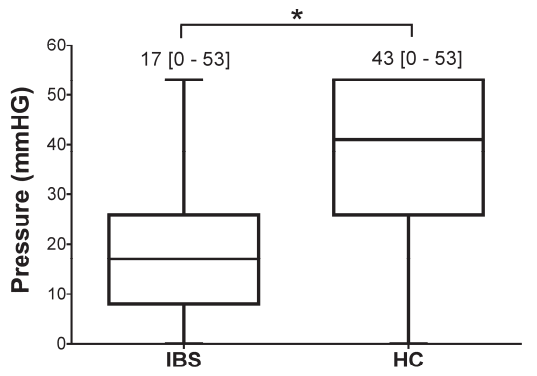

Figure 3.2 First sensation (a) and first pain (4) of IBS patients and HC following rectal distensions between 0 and $50 \mathrm{mmHg}$ above MDP. Values above the boxplots are median and range; ${ }^{*} P<0.01$.

\section{Visceral hypersensitivity}

In Table 3.1 the hypersensitivity percentages are shown for IBS patients and HC according to the three criteria described previously.

Using the mean pain threshold in $\mathrm{HC}$ minus $2 \mathrm{SD}$, a cut-off of VAS $>10 \mathrm{~mm}$ at or before pressure $23 \mathrm{mmHg}$ was found to detect visceral hypersensitivity. According to this criterion, hypersensitivity percentages were $34.9 \%$ in IBS and $6.6 \%$ in $\mathrm{HC}$.

Using the $10^{\text {th }}$ percentile of the pain threshold in $\mathrm{HC}$, the cut-off was found to be a VAS $>10 \mathrm{~mm}$ at or before pressure $11 \mathrm{mmHg}$. This resulted in $64.3 \%$ and $13.3 \%$ hypersensitivity in IBS patients and $\mathrm{HC}$, respectively.

Assessment of the optimal discriminative cut-off resulted in a VAS $\geq 20 \mathrm{~mm}$ at or before pressure $26 \mathrm{mmHg}$, according to the ROC curves. This cut-off has led to $63.5 \%$ of IBS patients and $10 \%$ of $\mathrm{HC}$ being hypersensitive. Table 3.2 shows the five pressure steps and VAS cut-off values with the highest discriminative capacity between IBS patients and $H C$, using AUC and LR+. The optimal cut-off point as described above was characterised by an AUC of 0.77 , a LR+ of 6.35 and a sensitivity, specificity and positive predictive value of $63 \%, 90 \%$, and $96 \%$, respectively.

According to the optimal discriminative cut-off, we calculated the number of hypersensitive patients among the different IBS subtypes. In IBS-D, 58.7\% of the patients were hypersensitive, whereas in IBS-C and IBS-M and IBS-U hypersensivity occurred in $67.6 \% ; 62.5 \%$ and $70.1 \%$ of the patients, respectively. These were not significantly different $(P=0.34)$, neither were the percentages of hypersensitive male vs. female patients ( $59.1 \%$ vs. $58.3 \%$, respectively; $P=0.28)$. 
Table 3.1 Visceral hypersensitivity in IBS patients and HC, based on I. mean pain threshold in healthy controls minus 2 times the standard deviation; II. $10^{\text {th }}$ percentile of the mean pain threshold in healthy controls and III. discriminative optimum between IBS patients and HC, based on pain perception scores

\begin{tabular}{lcc}
\hline Criterion & IBS patients (\%) & Healthy controls (\%) \\
\hline Mean pain threshold in HC - 2 SD & 34.9 & 6.6 \\
$10^{\text {th }}$ percentile of mean pain threshold in HC & 64.3 & 13.3 \\
Discriminative optimum between IBS patients and HC & 63.5 & 10.0 \\
\hline
\end{tabular}

Table 3.2 Five pressure steps and VAS cut-off values with the highest discriminative capacity for IBS patients and HC, based on area under ROC curve (AUC), sensitivity (SEN), specificity (SPE) positive predictive value (PPV) and positive likelihood ratio (LR+)

\begin{tabular}{lcccccc}
\hline Pressure $(\mathrm{mmHg})$ & VAS cut-off $(\mathrm{mm})$ & AUC & SEN & SPE & PPV & LR+ \\
\hline 20 & 10 & 0.75 & 0.60 & 0.90 & 0.96 & 5.95 \\
23 & 10 & 0.77 & 0.67 & 0.87 & 0.95 & 4,95 \\
26 & 20 & 0.77 & 0.63 & 0.90 & 0.96 & 6.35 \\
32 & 20 & 0.77 & 0.71 & 0.83 & 0.95 & 4.24 \\
38 & 25 & 0.77 & 0.70 & 0.83 & 0.95 & 4.19 \\
\hline
\end{tabular}

\section{Allodynia}

Based on the cut-off for first pain and cut-off for optimal discrimination, $11.1 \%$ of IBS patients and $6.6 \%$ of $\mathrm{HC}$ were found to be allodynic. The prevalence of allodynia did not differ between gender $(P=0.80)$ or IBS subtypes $(P=0.21)$.

\section{Discussion}

Aim of our study was to assess the optimal discriminative cut-off value for rectal hypersensitivity measured by a barostat, between IBS patients and healthy controls. The optimal cut-off was found to be at the pressure step of $26 \mathrm{mmHg}$ with a VAS-score $\geq 20 \mathrm{~mm}$, which resulted in $63.5 \%$ of IBS patients being hypersensitive, with a specificity of $90 \%$.

Our data confirm previous observations that IBS patients show increased perception to rectal distension ${ }^{6-8,10-12,26}$. Moreover, it has been demonstrated that the prevalence of hypersensitivity strongly depends on the method or barostat paradigm used. In the present study, IBS patients were found to have an increased visceral perception indicated by higher VAS-scores observed over the whole pressure distension range, compared to healthy controls. These results are supported by the lower perception thresholds for both first sensation and first pain in IBS patients versus healthy controls. 
However, these results only show that visceroperception is increased at group level and no definite conclusions can be drawn for individual subjects.

By applying different criteria for hypersensitivity, we have demonstrated that the percentages of hypersensitive IBS patients we found, reflect the wide range reported in literature ${ }^{8-13,26}$. Van der Veek et al. described that $33 \%$ of IBS patients were hypersensitive using the mean pain threshold minus 2SD in $H C^{8}$. The percentage hypersensitive patients that we observed when applying the same criterion, in effect $34.9 \%$, is in agreement with the data by van der Veek et al. However, when comparing the percentage hypersensitive IBS patients obtained by this criterion, with the percentage obtained by the $10^{\text {th }}$ percentile (64.3\%), the $10^{\text {th }}$ percentile appears to be a more acurate estimate of the actual hypersensitivity percentage (63.5\%), i.e. the percentage obtained by the discriminative optimum. One should realise that this holds true only for barostat measurements performed according to our protocol in our patient population.

As both these factors may affect the hypersensitivity percentage, it is important that institutes and motility labs that employ the barostat technique to define IBS subgroups with hypersensitivity, have standard protocols and should work on international standardization of barostat procedures.

Hypersensitivity consists of the two components: allodynia and hyperalgesia. This is the first study that has attempted to evaluate the allodynia component in IBS versus controls, which implicates pain perception to non-painful stimuli during minimal rectal distensions. This may provide a better insight in mechanisms involved in visceral hypersensitivity. Allodynia is hypothesized to be a result of low-threshold afferent neurons signalling to an already sensitised central nervous system (CNS), whereas hyperalgesia is primarily attributed to increased excitability of tissue nociceptive afferents and neurons in the CNS involved in nociceptive processing ${ }^{32}$. We found that the prevalence of allodynia in IBS patients, was low, namely $11.1 \%$, and it did not differ between IBS subtypes. Therefore sensitisation of the CNS seems to play only a minor role in the increased perceptive responses to distensions in IBS patients. This is supported by the observation that sensitisation by pre-protocol distension sequences does not affect visceral perception in IBS patients ${ }^{33}$.

It has to be noted that allodynia percentages were calculated based on the optimal discriminatory cut-off and first sensation, the latter of which was based on a study by van der Veek et al. ${ }^{8}$. For future studies, it is important to elaborate on the role of either allodynia or hyperalgesia, especially with respect to altered nerve signalling. Better understanding of hypersensitivity and its components in IBS allows patient selection for trials aimed at hypersensitivity. Nutritional (e.g. pre- and probiotics) and 
pharmaceutical (e.g. serotonergic) modulation of the enteric nervous system are likely to be of specific interest in this respect.

Compared to other reports on IBS, in our population the IBS-D subgroup is overrepresented $^{34}$. The reason for this is not known. We have shown that hypersensitivity is not affected by predominant bowel habit. IBS however, is a heterogenous disorder and future therapeutic approaches for IBS, perhaps should focus more on patients or subgroups that share identical pathophysiological mechanisms such as allodynia or hyperalgesia, but also psychological comorbidity. In this way, rectal barostat measurements may help to select IBS subgroups for tailored therapeutic interventions.

Increased sensory responses to rectal distensions in IBS have been researched for almost four decades ${ }^{35}$. Up to now, no consensus has been reached regarding the definition of visceral hypersensitivity. We have shown that visceral hypersensitivity and its interpretation are highly dependent on the methods and criteria used and may also differ between protocols. In order to generalise results obtained by barostat measurement we should take into account that standardization of the barostat protocol, perception assessment methods (e.g. Likert scaling versus VAS) and cut-off values for hypersensitivity are highly warranted. This will allow proper comparison of barostat data between studies.

In conclusion, we found that the optimal cut-off to detect hypersensitivity in IBS patients was a pressure of $26 \mathrm{mmHg}$ with a VAS-score $\geq 20 \mathrm{~mm}$, as assessed by our barostat protocol. It is recommended that other research institutes determine their optimal cut-off in order to detect hypersensitivity in IBS patients. 


\section{References}

1. Thompson WG, Longstreth GF, Drossman DA, Heaton KW, Irvine EJ, Muller-Lissner SA. Functional bowel disorders and functional abdominal pain. Gut. 1999;45 Suppl 2:I143-7.

2. Drossman DA, Camilleri M, Mayer EA, Whitehead WE. AGA technical review on irritable bowel syndrome. Gastroenterology. 2002;123:2108-31.

3. Azpiroz F. Hypersensitivity in functional gastrointestinal disorders. Gut 2002;51 Suppl 1:i25-8.

4. Camilleri M. Testing the sensitivity hypothesis in practice: tools and methods, assumptions and pitfalls. Gut. 2002; 51 Suppl 1: i34-40.

5. Camilleri M, Coulie B, Tack JF. Visceral hypersensitivity: facts, speculations, and challenges. Gut. 2001; 48:125-31.

6. Penning C, Steens J, van der Schaar PJ, Kuyvenhoven J, Delemarre JB, Lamers CB, Masclee AA. Motor and sensory function of the rectum in different subtypes of constipation. Scand J Gastroenterol 2001;36:32-38.

7. Steens J, Van Der Schaar PJ, Penning C, Brussee J, Masclee AA. Compliance, tone and sensitivity of the rectum in different subtypes of irritable bowel syndrome. Neurogastroenterol Motil. 2002;14:241-7.

8. van der Veek PP, Van Rood YR, Masclee AA. Symptom severity but not psychopathology predicts visceral hypersensitivity in irritable bowel syndrome. Clin Gastroenterol Hepatol. 2008;6:321-8.

9. Kanazawa M, Palsson OS, Thiwan SI, Turner MJ, van Tilburg MA, Gangarosa LM, Chitkara DK, Fukudo S, Drossman DA, Whitehead WE. Contributions of pain sensitivity and colonic motility to IBS symptom severity and predominant bowel habits. Am J Gastroenterol. 2008;103:2550-61.

10. Kuiken SD, Lindeboom R, Tytgat GN, Boeckxstaens GE. Relationship between symptoms and hypersensitivity to rectal distension in patients with irritable bowel syndrome. Aliment Pharmacol Ther. 2005;22:157-64.

11. Mayer EA, Gebhart GF. Basic and clinical aspects of visceral hyperalgesia. Gastroenterology. 1994;107: 271-93.

12. Posserud I, Syrous A, Lindstrom L, Tack J, Abrahamsson H, Simren M. Altered rectal perception in irritable bowel syndrome is associated with symptom severity. Gastroenterology. 2007;133:1113-23.

13. Mertz H, Naliboff B, Munakata J, Niazi N, Mayer EA. Altered rectal perception is a biological marker of patients with irritable bowel syndrome. Gastroenterology. 1995;109:40-52.

14. Kanazawa M, Hongo M, Fukudo S. Visceral hypersensitivity in irritable bowel syndrome. J Gastroenterol Hepatol. 2011;26 Suppl 3:119-21.

15. Simren M, Agerforz P, Bjornsson ES, Abrahamsson H. Nutrient-dependent enhancement of rectal sensitivity in irritable bowel syndrome (IBS). Neurogastroenterol Motil. 2007;19:20-9.

16. Simren $\mathrm{M}$, Abrahamsson $\mathrm{H}$, Bjornsson ES. Lipid-induced colonic hypersensitivity in the irritable bowel syndrome: the role of bowel habit, sex, and psychologic factors. Clin Ggastroenterol Hepatol. 2007;5: 201-8.

17. van der Veek PP, Steenvoorden M, Steens J, van der Schaar PJ, Brussee J, Masclee AA. Recto-colonic reflex is impaired in patients with irritable bowel syndrome. Neurogastroenterol Motil. 2007;19:653-9.

18. Caldarella MP, Milano A, Laterza F, Sacco F, Balatsinou C, Lapenna D, Pierdomenico SD, Cuccurullo F, Neri M. Visceral sensitivity and symptoms in patients with constipation- or diarrhea-predominant irritable bowel syndrome (IBS): effect of a low-fat intraduodenal infusion. Am J Gastroenterol. 2005;100:383-9.

19. Ludidi S, Conchillo JM, Keszthelyi D, Koning CJ, Vanhoutvin SA, Lindsey PJ, Leufkens AM, Kruimel JW, Jonkers DM, Masclee AA. Does meal ingestion enhance sensitivity of visceroperception assessment in irritable bowel syndrome? Neurogastroenterol Motil. 2012;24:47-53, e3.

20. Delvaux M, Louvel D, Mamet JP, Campos-Oriola R, Frexinos J. Effect of alosetron on responses to colonic distension in patients with irritable bowel syndrome. Aliment Pharmacol Ther. 1998;12:849-55.

21. Sagami Y, Shimada Y, Tayama J, Nomura T, Satake M, Endo Y, Shoji T, Karahashi K, Hongo M, Fukudo S. Effect of a corticotropin releasing hormone receptor antagonist on colonic sensory and motor function in patients with irritable bowel syndrome. Gut. 2004;53:958-64. 
22. Gecse K, Róka R, Ferrier L, Leveque M, Eutamene H, Cartier C, Ait-Belgnaoui A, Rosztóczy A, Izbéki F, Fioramonti J, Wittmann T, Bueno L. Increased faecal serine protease activity in diarrhoeic IBS patients: a colonic lumenal factor impairing colonic permeability and sensitivity. Gut. 2008;57:591-9.

23. Kuiken SD, Klooker TK, Tytgat GN, Lei A, Boeckxstaens GE. Possible role of nitric oxide in visceral hypersensitivity in patients with irritable bowel syndrome. Neurogastroenterol Motil. 2006;18:115-22.

24. Poitras P, Riberdy Poitras M, Plourde V, Boivin M, Verrier P. Evolution of visceral sensitivity in patients with irritable bowel syndrome. Dig Dis Sci. 2002;47:914-20.

25. Jiang W, Adam IJ, Kitsanta P, Tiernan J, Hill C, Shorthouse A, Grundy D. 'First-in-man': characterising the mechanosensitivity of human colonic afferents. Gut. 2011;60:281-2.

26. Bouin $M$, Plourde $V$, Boivin $M$, Riberdy $M$, Lupien $F$, Laganière $M$, Verrier $P$, Poitras $P$. Rectal distention testing in patients with irritable bowel syndrome: sensitivity, specificity, and predictive values of pain sensory thresholds. Gastroenterology. 2002;122: 1771-7.

27. Vanhoutvin SA, Troost FJ, Kilkens TO, Lindsey PJ, Hamer HM, Jonkers DM, Venema K, Brummer RJ. The effects of butyrate enemas on visceral perception in healthy volunteers. Neurogastroenterol Motil. 2009;21:952-e76.

28. Piche $M$, Arsenault $M$, Poitras $P$, Rainville $P$, Bouin $M$. Widespread hypersensitivity is related to altered pain inhibition processes in irritable bowel syndrome. Pain. 2010;148:49-58.

29. Agrawal A, Houghton LA, Lea R, Morris J, Reilly B, Whorwell PJ. Bloating and distention in irritable bowel syndrome: the role of visceral sensation. Gastroenterology. 2008;134:1882-9.

30. Ng C, Malcolm A, Hansen R, Kellow JE. Distension technique influences the relationship between colonic and rectal hypersensitivity in irritable bowel syndrome. Neurogastroenterol Motil. 2006;18: 206-10.

31. Darfeuille-Michaud A, Neut C, Barnich N, Lederman E, Di Martino P, Desreumaux P, Gambiez L, Joly B, Cortot A, Colombel JF. Presence of adherent Escherichia coli strains in ileal mucosa of patients with Crohn's disease. Gastroenterology. 1998;115:1405-13.

32. Koltzenburg M, Lundberg LE, Torebjork HE. Dynamic and static components of mechanical hyperalgesia in human hairy skin. Pain. 1992;51:207-19.

33. Hammer HF, Phillips SF, Camilleri M, Hanson RB. Rectal tone, distensibility, and perception: reproducibility and response to different distensions. Am J Physiol. 1998;274:G584-90.

34. Engsbro AL, Simren M, Bytzer P. Short-term stability of subtypes in the irritable bowel syndrome: prospective evaluation using the Rome III classification. Aliment Pharmacol Ther. 2012;35:350-9.

35. Ritchie J. Pain from distension of the pelvic colon by inflating a balloon in the irritable colon syndrome. Gut. 1973;14:125-32. 


\section{Chapter 4}

\section{Markers for visceral hypersensitivity in patients with irritable bowel syndrome}

S Ludidi*, Z Mujagic*, D Jonkers, D Keszthelyi, M Hesselink, J Kruimel, J Conchillo, A Masclee

* Both authors contributed equally to the manuscrip

Neurogastroenterol Motil - Epub ahead of print 


\section{Abstract}

\section{Background}

Irritable bowel syndrome (IBS) is a heterogenous disorder with visceral hypersensitivity as important hallmark. It is not known whether IBS patients with visceral hypersensitivity have different epidemiological and clinical characteristics compared to IBS patients without visceral hypersensitivity. Aim of our study was to compare in detail a large group of hyper- versus normosensitive IBS patients with respect to epidemiological and clinical characteristics.

\section{Methods}

IBS patients (Rome III criteria) have been recruited for a large-scale cohort study. All patients form this cohort that underwent a rectal barostat procedure were included and allocated based on those with and without visceral hypersensitivity. Patient demographics, and symptoms were collected using questionnaires (GSRS, HADS, SF-36) and a 14-day symptom diary for IBS-related symptoms. A multivariate logistic regression model was used to identify risk markers for having visceral hypersensitivity,

\section{Key results}

Ninety-five normosensitive and 93 hypersensitive IBS patients participated in this study. Hypersensitive patients had significantly higher scores for GSRS abdominal pain $(P<0.05)$, indigestion, reflux and constipation syndrome (all $P<0.01$ ) and IBS-symptom intensity, discomfort, (both $P<0.05)$ and mean symptom composite score $(P<0.01)$. Age, female sex and the use of SSRI medication were significantly different between the normo- and the hypersensitive IBS patients. However, after adjustment for other risk markers, only increasing age was found to be significantly associated with lower odds for having hypersensitivity (OR 0.97 ( $95 \% \mathrm{Cl}: 0.94 ; 0.99)$ ).

\section{Conclusion \& inferences}

Apart from more severe symptomatology, hypersensitive IBS patients are characterized by significantly younger age compared to normosensitve IBS patients. 


\section{Introduction}

Irritable bowel syndrome (IBS) is a functional disorder of the gastrointestinal (GI) tract and is characterized by altered bowel habits, associated with abdominal pain. The diagnosis is based on Rome III criteria, and includes four predominant subtypes i.e. IBS with diarrhea (IBS-D), constipation (IBS-C), mixed (IBS-M) and the unspecified subtype (IBS-U).

Patients with IBS have a heterogeneous phenotypical appearance. A multifactorial pathophysiology is assumed to be key in this diversity. It has been hypothesized that increased visceral perception or visceral hypersensitivity is a major pathophysiological characteristic involved in the etiology of IBS patients. A substantial subset of up to $60 \%$ of patients with IBS is characterized by visceral hypersensitivity, which is therefore considered an important hallmark of IBS ${ }^{1,2}$.

Using the rectal barostat, mechanical balloon distensions are applied to the rectum, allowing to discriminate between patients with and without visceral hypersensitivity ${ }^{1}$. Visceral hypersensitivity comprises both hyperalgesia (increased responses to painful stimuli) and allodynia (painful responses to non-noxious stimuli). In addition, it has been suggested that IBS patients with visceral hypersensitivity also exhibit increased responses to somatic stimuli, pointing to a more generalized (somatic and visceral) hypersensitivity in these patients ${ }^{3,4}$. These findings however, have not been confirmed by others ${ }^{5}$. Since visceral hypersensitivity is frequent in IBS and increased visceroperception in IBS patients is associated with higher severity of self-reported symptoms ${ }^{6,7}$, patients with visceral hypersensitivity may represent a separate entity within the IBS population, not only from a pathophysiological but also from a clinical perspective. However, a more detailed characterization of potential differences in clinical features between patients with and without visceral hypersensitivity is currently lacking ${ }^{3,8}$.

The aim of the present study was therefore to compare demographical, psychological, lifestyle, as well as medication-related risk markers for having visceral hypersensitivity in patients with IBS. We hypothesize that the female sex and severity of psychological and gastro-intestinal symptoms are significantly associated with having visceral hypersensitivity. 


\section{Materials and methods}

The present study is part of a large prospective cohort study on the phenotypical and genotypical characterization of patients with IBS, of which 126 patients had participated in a previous study by our group ${ }^{2}$.

Subjects with IBS included in this study were seen at our outpatient department from the Maastricht University Medical Center+ between September 2009 and June 2013. All secondary and tertiary referral IBS patients were also seen at the motility research unit at the Maastricht University Medical Center+.

Prior to participation, subjects had signed an informed consent and the study protocol had been approved by the Maastricht University Medical Centre+ Committee of Ethics and was executed according to the Declaration of Helsinki $\left(59^{\text {th }}\right.$ general assembly of the WMA, Seoul, South Korea, Oct. 2008). The study has been registered in the US National Library of Medicine (http://www.clinicaltrials.gov, NCT00775060).

\section{Patients}

Between September 2009 and June 2013, IBS patients visiting the gastroenterology outpatient clinic oft Maastricht University Medical Centre, were asked to participate. Patients with an organic disease cause were excluded. All subjects that were included complied with Rome III criteria for IBS. The present study only included patients from our IBS cohort who were willing to undergo a rectal barostat procedure for the assessment of visceral perception. Patients could not participate if they were unable to stop medication that can influence gastrointestinal motility or perception for at least 3 days prior to the test.

Furthermore, all patients that participated in the IBS cohort had filled out questionnaires on demographics, symptoms, medical and lifestyle factors. In addition, they kept a symptom diary for 14 days.

\section{Rectal barostat}

Participants arrived in the hospital after an overnight fast. Rectal perception was measured using an electronic barostat (Distender II; G\&J Electronics, Toronto, ON, Canada, part: C7-CB-R) and a balloon of non-compliant material (Mui Scientific, Missisauga, ON, Canada, part: C7-2CB-R) was lubricated with KY-gel (Johnsson \& Joshnsson, Longhorne, PA, USA). The procedure was executed according to our standard protocol as previously described ${ }^{9}$. Accordingly, pain was scored, using a visual analogue scale (VAS) ranging between 0-100 $\mathrm{mm}$. For subjects that did not complete the full protocol, VAS-scores for the remaining pressure steps were replenished, using the highest VAS-score achieved over the protocol thus far. Cut-off for visceral 
hypersensitivity was defined a VAS score of $\geq 20 \mathrm{~mm}$ at pressure step $26 \mathrm{mmHg}$. Furthermore, the percentage of subjects with allodynia, i.e. early pain perception in response to non-noxious stimuli, was calculated based on VAS $>10 \mathrm{~mm}$

prior to the pressure step indicative for visceral hypersensitivity (i.e. $26 \mathrm{mmHg}$ ) as previously described by our group ${ }^{2}$.

\section{Questionnaires}

Within a period of 2 weeks prior to the rectal barostat, questionnaires on e.g. demographics, lifestyle factors (smoking and drinking behavior), stress events in relation to the presence of symptoms, symptom onset and the use of medication were completed. Furthermore, the presence of GI symptoms was assessed using the validated gastrointestinal symptom rating scale (GSRS), consisting of 16 items clustered into 5 major Gl syndromes: abdominal pain (AP), reflux syndrome (RS) diarrhea syndrome (DS), indigestion syndrome (IS), constipation syndrome (CS) $)^{10}$. Anxiety and depression were assessed using the hospital anxiety and depression scale (HADS). A score $\geq 8$ on either the anxiety or depression scale were indicative for the presence of anxiety or depressive symptoms, respectively ${ }^{11,12}$. The RAND-36 questionnaire, which is the validated Dutch version of the Medical Outcomes Study 36-item Short Form Health Survey (SF-36) ${ }^{13}$, was used to assess generic health-related quality of life (QoL). The SF36 consists of 8 domains, all ranging between $0-100$, of which a mental (QoL-MCS) and physical (QoL-PCS) composite score can be derived ${ }^{14,15}$. Finally, IBS-related symptoms of discomfort, abdominal pain, bloating, flatulence, nausea as well as perceived intensity of symptoms were scored on a 5-point Likert-scale between 1-5 for 14 consecutive days prior to the rectal barostat procedure. Subsequently, the mean, i.e. per day, symptom composite score (MSS) was calculated (range: 1-5), based on sum of individual symptoms.

\section{Data and statistical analysis}

Based on barostat data, patients were defined as normosensitive (IBS NORM $_{\text {) or }}$ hypersensitive $\left(\mathrm{IBS}_{\mathrm{HYP}}\right)$ to rectal distension. Data were analyzed for normality using a normal probability plot. Subsequently, descriptives are presented as mean or percentages as appropriate, for the whole group of IBS patients (IBS TOTAL $_{\text {TA }}$ ) as well as for the two IBS subgroups. Dichotomous data were analyzed using a Chi-square test, continuous and ordinal data were analyzed using an independent t-test.

Next, a multivariate logistic regression model was used to identify risk markers for having visceral hypersensitivity. The following variables were included in the model: I. demographical characteristics (i.e. age, sex, BMI, IBS-subtype and symptom duration) II. psychological symptoms (i.e. anxiety and depressive symptoms) III. lifestyle factors (i.e. 
smoking history and use of alcohol) and IV. the use of medication (i.e. NSAID and SSRI). Adjusted data were shown as odds ratios (OR) and 95\% confidence intervals (Cl). Finally, a Pearson's correlation test was performed to correlate visceroperception with symptoms and age.

Statistical analyses were performed using IBM SPSS Statistics version 20.0 (IBM Statistics for Macintosh, Chicago IL) and a two-sided $P<0.05$ was considered statistically significant.

\section{Results}

\section{Demographical characteristics and visceroperception}

A total of 188 IBS patients underwent a rectal barostat procedure, of which 93 (49.5\%) patients were found to be hypersensitive. In Figure 4.1 VAS-scores for pain perception in $\mathrm{IBS}_{\mathrm{NORM}}$ and $\mathrm{IBS}$ HYP patients are shown. Of the $\mathrm{IBS}_{\mathrm{NORM}}$ patients, $10.8 \%$ showed allodynia compared to. $89.2 \%$ in the $\mathrm{IBS}_{\mathrm{HYP}}$ patients $(P<0.001)$.

A detailed description of the patients' demographical characteristics, psychological symptoms, lifestyle factors and medication use is given in Table 4.1. Compared to $\mathrm{IBS}_{\mathrm{NORM}}, \mathrm{IBS}_{\mathrm{HYP}}$ patients were significantly younger $(36.9 \pm 1.62$ vs. $45.8 \pm 1.71$ years; $P<0.001)$ and more frequently female $(81.7 \%$ vs. $66.0 \% ; P<0.05)$. No significant differences were observed in BMI, IBS subtype or symptom duration.

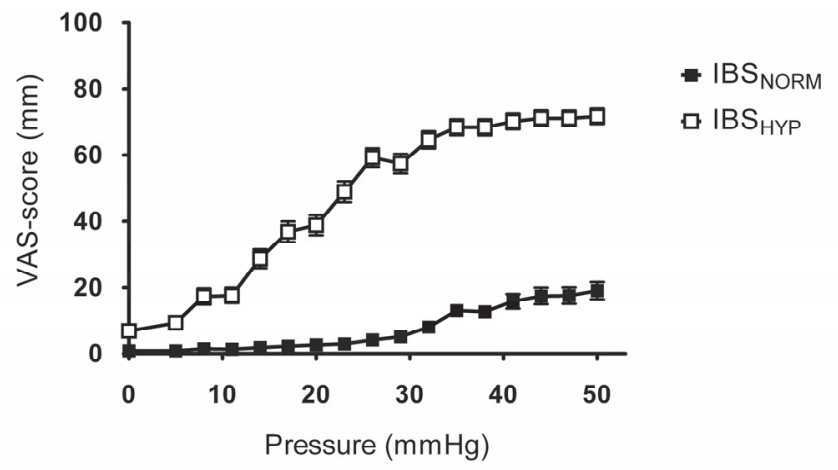

Figure 4.1 Visceroperception barostat data of normosensitive (IBS NORM $_{\text {) }}$ and hypersensitive (IBS HYP $_{\text {I }}$ IBS patients for pain, expressed as visual analogue scale (VAS) scores versus intra-balloon pressure in $\mathrm{mmHg}$. 
Table 4.1 Patient characteristics based on demographical characteristics, psychological symptoms, lifestyle factors and the use of medication in all patients $(N=188)$ that underwent a barostat procedure and split for patients without $(\mathrm{N}=95)$ and with $(\mathrm{N}=93)$ visceral hypersensitivity.

\begin{tabular}{lccc}
\hline Parameter & IBS & & \\
TOTAL & IBS $_{\text {NORM }}$ & IBS $_{\text {HYP }}$ \\
\hline I. Demographical characteristics & & & \\
Age (years; mean \pm SEM) & $41.4 \pm 0.03$ & $45.8 \pm 1.71$ & $36.9 \pm 1.62 * * *$ \\
Female sex (\%) & 73.9 & 66.0 & $81.7 *$ \\
BMI & $24.4 \pm 0.33$ & $25.4 \pm 0.47$ & $23.4 \pm 0.48$ \\
IBS subtype (\%) & & & \\
IBS-D & 35.1 & 40.4 & 30.1 \\
IBS-C & 20.2 & 17.0 & 23.7 \\
IBS-M & 37.8 & 35.1 & 40.9 \\
IBS-U & 6.9 & 7.4 & 5.4 \\
Duration of symptoms (years) & $12.1 \pm 1.13$ & $11.7 \pm 1.60$ & $12.5 \pm 1.32$ \\
Post-infectious IBS (\%) & 21.3 & 21.5 & 21.0 \\
II. Psychological symptoms - anxiety and depressive symptoms (HADS) & & \\
Depressive symptoms (\%) & 20.7 & 17.9 & 23.7 \\
Anxiety symptoms (\%) & 39.4 & 40.0 & 38.7 \\
III. Lifestyle parameters & & & 49.5 \\
Positive smoking history (\%) & 48.4 & 47.4 & 5.4 \\
Alcohol intake > 15/week (\%) & 6.9 & 8.4 & \\
IV. Use of medication 2 weeks prior to participation & & & 20.4 \\
PPI (\%) & 22.9 & 25.3 & 9.6 \\
NSAID (\%) & 12.2 & 14.7 & 20.4 \\
SSRI (\%) & 11.2 & 6.3 & 9.7 \\
Motility +drugs (\%) & 18.1 & 15.8 & 7.4 \\
Motility -drugs (\%) & 8.5 & & \\
\hline
\end{tabular}

IBS TOTAL: Whole group of IBS patients; IBS $_{\text {NORM: Normosensitve BIS patients; IBS }}$ HYP: Hypersensitive IBS patients; PI: post-infectious. $* P<0.05$ vs. IBS $_{\text {NORM }} ; * * P<0.001$ vs. IBS $_{\text {NORM }}$.

When analyzing pain perception in more detail, we found that $52.1 \%(\mathrm{~N}=98)$ of the $\mathrm{IBS}_{\text {TOTAL }}$ patients were allodynic, i.e. experienced pain at low pressure $(<26 \mathrm{mmHg}$ ). Similar to hypersensitive patients, patients with allodynia were significantly younger versus the non-allodynic patients $(36.5 \pm 1.64$ vs. $46.0 \pm 1.79 ; P<0.001)$ as well as more often of the female sex (78.5\% vs. $68.9 \% ; P<0.05)$. No other differences were observed between patients with and without allodynia.

\section{Risk markers for having visceral hypersensitivity}

In Table 4.2 risk markers for visceral hypersensitivity are presented. After adjustment for other potential risk markers, only increasing age was found to be significantly associated with lower odds for having hypersensitivity (OR 0.97 (95\% Cl: $0.94 ; 0.99)$ ). Also, age was found to correlate significantly though weakly with intensity of pain (VAS) at pressure step $26 \mathrm{mmHg}$ and age $(\mathrm{R}=-0.253 ; P<0.01)$. In addition, medication use showed higher but not statistically significant odds for having visceral hypersensitivity in patients using SSRIs (OR: 2.6 (95\% Cl: 0.90; 7.52)). 
Table 4.2 Odds ratios for having visceral hypersensitivity in comparison to normosensitvity based on demographical characteristics, psychological symptoms, lifestyle factors and the use of medication. $95 \% \mathrm{Cl}$ : $95 \%$ confidence interval.

\begin{tabular}{lccc}
\hline Parameter & Odds ratio & $95 \% \mathrm{Cl}$ & $P$-value \\
\hline I. Demographical characteristics & & & \\
$\quad$ Age (years) & 0.97 & $0.94 ; 0.99$ & 0.004 \\
Sex (female reference) & 0.56 & $0.26 ; 1.20$ & 0.135 \\
BMI (kg/m²) & 0.94 & $0.87 ; 1.02$ & 0.134 \\
Symptom duration (years) & 1.02 & $0.97 ; 1.05$ & 0.289 \\
IBS subtype & 1.08 & $0.78 ; 1.50$ & 0.629 \\
II. Psychological symptoms & & & \\
$\quad$ Depressive symptoms & 1.32 & $0.54 ; 3.18$ & 0.543 \\
Anxiety symptoms & 0.89 & $0.42 ; 1.90$ & 0.766 \\
III. Lifestyle factors & & & 0.452 \\
Positive smoking history & 1.30 & $0.66 ; 2.55$ & 0.541 \\
$\quad$ Use of alcohol $>15$ units/ week & 0.66 & $0.17 ; 2.52$ & \\
IV. Use of medication & & & 0.459 \\
$\quad$ Use of NSAID & 0.68 & $0.24 ; 1.90$ & 0.077 \\
$\quad$ Use of SSRI & 2.60 & $0.90 ; 7.52$ & \\
\hline
\end{tabular}

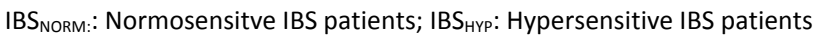

\section{Symptom scores and quality of life}

Scores for symptoms according to the GSRS and the IBS symptom diary, as well as QoL scores are given in Table 4.3. GSRS symptom scores for abdominal pain, reflux syndrome, indigestion syndrome and constipation syndrome were significantly increased in the IBS $\mathrm{HYP}_{\mathrm{HP}}$ when compared to the IBS $\mathrm{BSRM}_{\mathrm{NO}}$ group. Resulting from the symptom diary, $\mathrm{IBS}_{\mathrm{HYP}}$ patients also showed significantly higher scores for discomfort, abdominal pain and intensity of symptoms. As a result, MSS was significantly increased

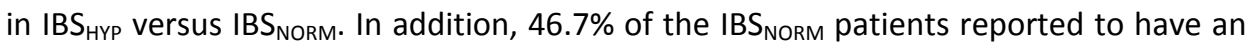
exacerbation of symptoms with stress versus $53.3 \%$ of the IBS $S_{\text {HYP }}$ patients $(P=0.41)$.

Correlating GSRS symptom scores with visceroperception (i.e. VAS-score at pressure step $26 \mathrm{mmHg}$ ), significantly positive, though weak correlations (all $R<0.25$ ) were observed for reflux syndrome, diarrhea syndrome, indigestion syndrome and constipation syndrome $(P<0.05)$. No correlation was observed between GSRS for abdominal pain. Diary symptoms scores for discomfort $(P<0.01)$ abdominal pain $(P<0.01)$, intensity of symptoms $(P<0.01)$ and MSS $(P<0.05)$ showed significant correlations with visceroperception scores (all $R<0.28$; data not shown).

Regarding quality of life, no differences were observed between IBS ${ }_{\text {NORM }}$ and IBS HYP $_{\text {with }}$ respect to QoL-PCS and QoL-MCS (Table 4.3). 
Table 4.3 Symptoms and quality of life in patients without $(\mathrm{N}=95)$ and with $(\mathrm{N}=93)$ visceral hypersensitivity $\left(\right.$ IBS $_{\text {NORM }}$ and IBS ${ }_{\text {HYP }}$, respectively).

\begin{tabular}{lll}
\hline Parameter & $\mathrm{IBS}_{\mathrm{NORM}}$ & $\mathrm{IBS}_{\mathrm{HYP}}$ \\
\hline GSRS syndrome (Mean \pm SEM) & & \\
Abdominal pain & $3.15 \pm 0.13$ & $3.57 \pm 0.13^{*}$ \\
$\quad$ Reflux syndrome & $1.73 \pm 0.13$ & $2.29 \pm 0.13^{* *}$ \\
Diarrhoea syndrome & $3.26 \pm 0.18$ & $3.54 \pm 0.17$ \\
Indigestion syndrome & $3.69 \pm 0.13$ & $4.24 \pm 0.15^{* *}$ \\
Constipation syndrome & $2.99 \pm 0.14$ & $3.62 \pm 0.17^{* *}$ \\
SF-36 (Mean \pm SEM) & & \\
QoL-PCS & $40.90 \pm 1.18$ & $39.01 \pm 1.14$ \\
QoL-MSC & $48.48 \pm 1.20$ & $46.33 \pm 1.16$ \\
Symptom scores - 14 day average (Mean \pm SEM) & & \\
Discomfort & $2.31 \pm 0.09$ & $2.61 \pm 0.80^{*}$ \\
Abdominal pain & $2.11 \pm 0.10$ & $2.52 \pm 0.10^{* *}$ \\
Bloating & $2.02 \pm 0.12$ & $2.32 \pm 0.11$ \\
Flatulence & $2.21 \pm 0.11$ & $2.24 \pm 0.10$ \\
Nausea & $1.52 \pm 0.81$ & $1.79 \pm 0.11$ \\
Intensity of symptoms & $2.38 \pm 0.10$ & $2.72 \pm 0.09^{*}$ \\
Mean symptom composite score & $12.49 \pm 0.48$ & $14.2 \pm 0.40^{* *}$ \\
\hline
\end{tabular}

IBS NORM: $_{\text {: Normosensitve IBS patients; IBS }}$ HYP: Hypersensitive IBS patients; GSRS: gastrointestinal symptom rating scale; SF-36: 36-item short form health survey; PCS: physical quality of life composite score; MCS: mental quality of life composite score. ${ }^{*} P<0.05$ vs. IBS $\mathrm{IORM}^{* *} P<0.01 \mathrm{vs}$. IBS $\mathrm{IORM}^{* * *} P<0.001 \mathrm{vs}$. IBS $\mathrm{NORM}$.

\section{Effect of psychological status on symptoms}

GSRS symptoms abdominal pain and indigestion syndrome were found significantly affected by HADS-A scores ( $P=0.02$ and 0.03 , respectively), but not by HADS-D scores. IBS-diary symptom scores for discomfort $(P=0.01)$, abdominal pain and symptom intensity and MSS (all $P=0.04$ ) on the other hand, were found to be significantly affected by the HADS-D scores, but not by HADS-A. Correcting for HADS-A and HADS-D however, did not alter the results as symptom scores for the abovementioned parameters remained significantly increased in the hyper- versus the normosensitive IBS patients

\section{Discussion}

We have demonstrated that visceral hypersensitivity in patients with IBS is associated with younger age, female sex and use of SSRI medication. Having visceral hypersensitivity was also associated with significantly increased GSRS scores and IBSrelated symptom scores, compared to patients without visceral hypersensitivity. In the multivariate analysis, only age remained a statistically relevant marker of visceral hypersensitivity. 
Hypersensitive patients were more frequently of younger of age when compared to normosensitive IBS patients. Given the fact that symptom duration was not significantly different among hyper- and normosensitive patients, the patients with visceral hypersensitivity have presented at a younger age. Previous data point to a decline in visceral pain perception with aging, showing higher pain thresholds to rectal distension in older (i.e. $86 \pm 4$ years) compared to younger (i.e. $26 \pm 1$ years) healthy subjects ${ }^{16}$. Pain perception to lower range pressure distensions points to an allodynic type response. Most patient in the hypersensitive group also had allodynia. It has been observed that ageing results in a decrease in the number of sensory neurons in the intestine ${ }^{17,18}$. This may account, at least in part, for the difference in age between the hyper- and normosensitive group. Anticipatory responses on the other hand, may also contribute. Pain sensitivity in general, but also visceral hypersensitivity has been associated with an increased tendency to report symptoms ${ }^{19}$, pointing to involvement of central mechanisms, e.g. altered coping and potential and psychological factors such as anxiety in symptom presentation in $\mathrm{IBS}^{20,21}$. In line with these observations, we recently demonstrated that dysfunctional cognitions, which are related to coping, independently affect IBS symptomatology ${ }^{22}$. Accordingly, a decreased perceptual response to stimuli may occur over time. As previously described this is most likely due to adapting coping strategies and habituation ${ }^{20,23}$. Although desensitization over time could also have accounted for the significant age discrepancy in our study population (i.e. normo- versus hypersensitives), symptom duration was not different between the two groups. Therefore, we assume that hypersensitive patients have a different underlying pathophysiology, not necessarily only pain-associated anticipation as proposed by Dorn et al. with altered coping mechanisms over time ${ }^{19}$.

The hypersensitive IBS patients were not only significantly younger but also more frequently female. Concerning gender differences, some groups have observed higher responses to painful stimuli (i.e. lower threshold or tolerance) in women compared to men $^{24}$, which may account for the female predominance in the hypersensitive group. The influence of sex hormones may give rise to such differences between women and men. Accounting for menstrual cycle when analyzing visceral sensitivity could have given better insight in the potential association between sex hormones and visceral sensitivity. Unfortunately, these data were not available in our population from daily clinical practice. After correction for potentially confounding factors in the multivariate model female sex was no longer found to be a risk marker for visceral hypersensitivity in our IBS population.

Alcohol intake and smoking are well-known lifestyle risk factors associated with several gastrointestinal and liver diseases and carcinogenesis. With regard to IBS, several 
studies have reported that lifestyle factors are associated with severity of symptoms ${ }^{25-28}$.

Previously, we have observed that after acute alcohol intake intestinal permeability in healthy subjects increases ${ }^{29}$. Increased intestinal permeability has been related to symptom severity and may contribute to visceral hypersensitivity ${ }^{30}$. We evaluated whether alcohol and smoking behavior were associated with visceral perception, but could not find significant associations between these risk markers and visceral pain perception.

A subset of IBS patients develops symptoms following acute gastroenteritis ${ }^{31}$. This specific form of IBS is referred to as post-infectious (PI) IBS. PI-IBS is typically characterized by low-grade inflammation ${ }^{32}$, which may contribute to the development of pain symptoms ${ }^{33}$. In the present study, we used a questionnaire as a screening tool for IBS including questions related to PI-IBS. No differences were observed in PI-IBS prevalence between hyper and normosensitive patients (20 vs. $21 \%$, respectively). Percentages we found are within the range of those observed in other studies and cover the range in which patients report IBS symptom onset, following acute gastroenteritis $^{32,34}$.

Anxiety and depression are more prevalent among IBS patients compared to control subjects. Several studies have shown that $20-30 \%$ of IBS patients have depressive symptoms and $15-45 \%$ anxiety symptoms ${ }^{22,35}$. Associations have been found between anxiety and depression and the severity of reported symptoms in IBS ${ }^{36-38}$. Previously, Dorn et al. found that IBS patients with higher scores on psychometric scales for anxiety and depression have an increased tendency to report symptoms during the barostat procedure $^{19}$. Although we compared a substantial number of IBS patients, psychological distress as measured with the HADS was equally distributed among patients with and without visceral hypersensitivity. Neither did absolute scores for HADS-A and HADS-D differ between the normosensitive versus the hypersensitive patients nor did intensity of pain correlate with HADS score (data not shown). Based on our data we assume that anxiety and depression are likely to be associated with having IBS per se, but not with having visceral hypersensitivity.

Concerning medication, use of SSRI was more frequent among the hypersensitive compared to the normosensitive patients. This was statistically significant in the univariate analysis $(16.1 \%$ vs. $6.3 \%$, respectively; $P<0.05)$ but not in the multivariate analysis $(P=0.077)$. SSRI medication is more frequently prescribed among the IBS population compared to the general population due to an increased prevalence of anxiety and depression in $\mathrm{IBS}^{22,35}$. On the other hand, SSRI's are also prescribed for control of abdominal symptoms, especially pain ${ }^{39}$. As symptoms are more pronounced 
in hypersensitive patients, as shown by GSRS and symptom diary scores, this may account for higher percentage of SSRI users in the hypersensitive group.

Hypersensitive IBS patients represent a patient group with more pronounced symptoms during provocation (mechanical distension) with up to $90 \%$ also with early onset of pain, referred to as allodynia. Previous studies indicate that pain perception during provocation not necessarily correlates with symptom scores in daily life and Posserud et al. previously demonstrated that GSRS scores only moderately correlated with pain thresholds assessed by the rectal barostat ${ }^{7}$. Sabate et al. found no correlation between pain thresholds after rectal distensions and IBS symptom severity ${ }^{40}$. In a previous study by our group, we demonstrated that IBS symptoms were increased in patients with visceral hypersensitivity but not in those without hypersensitivity. ${ }^{6}$. In the present study also symptoms according to the GSRS (i.e. abdominal pain, constipation syndrome, reflux syndrome and indigestion syndrome) were significantly increased in the hypersensitive IBS patients. Of these symptoms, constipation, reflux and indigestion syndrome weakly correlated with visceral sensitivity thresholds. Also with regards to the IBS symptom diary scores, we only found weak correlations (i.e. for discomfort, abdominal pain, intensity of symptoms and mean symptom score) In this regard we should realize that the barostat is a symptom provocation test that measures threshold and intensity of pain in response to stimulation. With scores as GSRS and with diaries, reporting pain retrospectively (e.g. at the end of the day when using a diary) may give rise to recall bias. Although abdominal pain and indigestion syndrome (derived form the GSRS) were significantly affected by the presence of anxiety symptoms (HADS) and discomfort, abdominal pain, intensity of symptoms and MSS, were significantly affected by the presence of depressive symptoms, Correction for psychological comorbidity (HADS-A and HADS-D) did not alter the symptom scores in hypersensitive IBS patients, nor the difference between hyper- and normosensitive patients. This observation supports the concept that not only visceroperception but also altered psychological status affects symptom generation in IBS, supporting a role of the brain-gut axis in these patients.

In conclusion, we have compared a substantial group of IBS patients, with and without visceral hypersensitivity in search of patient characteristiscs and markers for visceral hypersensitivity. Our data show that IBS patients with visceral hypersensitivity are significantly younger and report significantly increased intensity of gastrointestinal symptoms, but no other characteristics or markers could be discerned. 


\section{References}

1. Mertz H, Naliboff B, Munakata J, Niazi N, Mayer EA. Altered rectal perception is a biological marker of patients with irritable bowel syndrome. Gastroenterology. 1995;109: 40-52.

2. Ludidi S, Conchillo JM, Keszthelyi D, Van Avesaat M, Kruimel JW, Jonkers DM, Masclee AA. Rectal hypersensitivity as hallmark for irritable bowel syndrome: defining the optimal cutoff. Neurogastroenterol Motil. 2012;24:729-33.

3. Piche $M$, Arsenault $M$, Poitras $P$, Rainville $P$, Bouin $M$. Widespread hypersensitivity is related to altered pain inhibition processes in irritable bowel syndrome. Pain. 2010;148:49-58.

4. Zhou Q, Fillingim RB, Riley JL, 3rd, Malarkey WB, Verne GN. Central and peripheral hypersensitivity in the irritable bowel syndrome. Pain. 2010;148:454-61.

5. Whitehead WE, Holtkotter B, Enck P, Hoelzl R, Holmes KD, Anthony J, Shabsin HS, Schuster MM. Tolerance for rectosigmoid distention in irritable bowel syndrome. Gastroenterology. 1990;98:1187-92.

6. van der Veek PP, Van Rood YR, Masclee AA. Symptom severity but not psychopathology predicts visceral hypersensitivity in irritable bowel syndrome. Clin Gastroenterol Hepatol. 2008;6:321-8.

7. Posserud I, Syrous A, Lindstrom L, Tack J, Abrahamsson H, Simren M. Altered rectal perception in irritable bowel syndrome is associated with symptom severity. Gastroenterology. 2007;133:1113-23.

8. Zhou Q, Verne GN. New insights into visceral hypersensitivity--clinical implications in IBS. Nat Rev Gastroenterol Hepatol. 2011;8:349-55.

9. Ludidi S, Conchillo JM, Keszthelyi D, Koning CJ, Vanhoutvin SA, Lindsey PJ, Leufkens AM, Kruimel JW, Jonkers DM, Masclee AA. Does meal ingestion enhance sensitivity of visceroperception assessment in irritable bowel syndrome? Neurogastroenterol Motil. 2012;24:47-53, e3.

10. Svedlund J, Sjodin I, Dotevall G. GSRS--a clinical rating scale for gastrointestinal symptoms in patients with irritable bowel syndrome and peptic ulcer disease. Dig Dis Sci 1988;33:129-34.

11. Zigmond AS, Snaith RP. The hospital anxiety and depression scale. Acta Psychiatr Scand. 1983;67: 361-70.

12. Bjelland I, Dahl AA, Haug TT, Neckelmann D. The validity of the Hospital Anxiety and Depression Scale. An updated literature review. J Psychosom Res. 2002;52:69-77.

13. Zee vd. Het meten van de algemene gezondheidstoestand met de RAND-36, een handleiding [Assessment of general health status with the RAND-36; guidebook]. 1993.

14. Creed F, Ratcliffe J, Fernandez L, Tomenson B, Palmer S, Rigby C, Guthrie E, Read N, Thompson D. Health-related quality of life and health care costs in severe, refractory irritable bowel syndrome. Ann Intern Med. 2001;134:860-8.

15. Farivar SS, Cunningham WE, Hays RD. Correlated physical and mental health summary scores for the SF-36 and SF-12 Health Survey, V.I. Health Qual Life Outcomes 2007;5:54.

16. Lagier E, Delvaux M, Vellas B, Fioramonti J, Bueno L, Albarede JL, Frexinos J. Influence of age on rectal tone and sensitivity to distension in healthy subjects. Neurogastroenterol Motil. 1999;11:101-7.

17. Gabella G. Fall in the number of myenteric neurons in aging guinea pigs. Gastroenterology. 1989;96: 1487-93.

18. Meciano Filho J, Carvalho VC, de Souza RR. Nerve cell loss in the myenteric plexus of the human esophagus in relation to age: a preliminary investigation. Gerontology. 1995;41:18-21.

19. Dorn SD, Palsson OS, Thiwan SI, Kanazawa M, Clark WC, van Tilburg MA, Drossman DA, Scarlett Y, Levy RL, Ringel Y, Crowell MD, Olden KW, Whitehead WE. Increased colonic pain sensitivity in irritable bowel syndrome is the result of an increased tendency to report pain rather than increased neurosensory sensitivity. Gut. 2007;56:1202-9.

20. Aziz Q. Visceral hypersensitivity: fact or fiction. Gastroenterology. 2006;131:661-4.

21. Fichna J, Storr MA. Brain-Gut Interactions in IBS. Front Pharmacol. 2012;3:127.

22. Thijssen AY, Jonkers DM, Leue $C$, van der Veek PP, Vidakovic-Vukic M, van Rood YR, Clemens $\mathrm{CH}$, Masclee AA. Dysfunctional cognitions, anxiety and depression in irritable bowel syndrome. J Clin Gastroenterol. 2010;44:e236-41. 
23. Naliboff BD, Berman S, Suyenobu B, Labus JS, Chang L, Stains J, Mandelkern MA, Mayer EA. Longitudinal change in perceptual and brain activation response to visceral stimuli in irritable bowel syndrome patients. Gastroenterology 2006; 131: 352-365.

24. Unruh AM. Gender variations in clinical pain experience. Pain. 1996;65:123-67.

25. Dong L, Dingguo L, Xiaoxing X, Hanming L. An epidemiologic study of irritable bowel syndrome in adolescents and children in China: a school-based study. Pediatrics. 2005;116:e393-6.

26. Reding KW CK, Jarrett ME, Eugenio MD, Heitkemper MM. Relationship between patterns of alcohol consumption and gastrointestinal symptoms among patients with irritable bowel syndrome. Am J Gastroenterol. 2013;108:270-6.

27. Mearin F, Badía X, Balboa A, Baró E, Caldwell E, Cucala M, Díaz-Rubio M, Fueyo A, Ponce J, Roset M, Talley NJ. Irritable bowel syndrome prevalence varies enormously depending on the employed diagnostic criteria: comparison of Rome II versus previous criteria in a general population. Scand J Gastroenterol 2001;36:1155-61.

28. Li FX, Patten SB, Hilsden RJ, Sutherland LR. Irritable bowel syndrome and health-related quality of life: a population-based study in Calgary, Alberta. Can J Gastroenterol. 2003; 17: 259-263.

29. Elhaseen Elamin DJ, Freddy Troost, Jan Dekker, Ad Masclee. Tu1352 Moderate Dosage of Ethanol Increases Small and Large Intestinal Permeability in Healthy Volunteers. Gastroenterology. 2012; 142.

30. Zhou Q, Zhang B, Verne GN. Intestinal membrane permeability and hypersensitivity in the irritable bowel syndrome. Pain. 2009;146:41-6.

31. Ghoshal UC, Ranjan P. Post-infectious irritable bowel syndrome: the past, the present and the future. J Gastroenterol Hepatol. 2011;26 Suppl 3:94-101.

32. Spiller R, Lam C. An Update on Post-infectious Irritable Bowel Syndrome: Role of Genetics, Immune Activation, Serotonin and Altered Microbiome. J Neurogastroenterol Motil 2012;18:258-68.

33. Gecse K, Róka R, Ferrier L, Leveque M, Eutamene H, Cartier C, Ait-Belgnaoui A, Rosztóczy A, Izbéki F, Fioramonti J, Wittmann T, Bueno L. Increased faecal serine protease activity in diarrhoeic IBS patients: a colonic lumenal factor impairing colonic permeability and sensitivity. Gut. 2008;57:591-9.

34. Longstreth GF, Hawkey CJ, Mayer EA, Jones RH, Naesdal J, Wilson IK, Peacock RA, Wiklund IK. Characteristics of patients with irritable bowel syndrome recruited from three sources: implications for clinical trials. Aliment Pharmacol Ther 2001;15:959-64.

35. Lee S WJ, Ma YL, Tsang A, Guo WJ, Sung J. Irritable bowel syndrome is strongly associated with generalized anxiety disorder: a community study. Aliment Pharmacol Ther 2009;30:643-51.

36. Surdea-Blaga T, Baban A, Dumitrascu DL. Psychosocial determinants of irritable bowel syndrome. World journal of gastroenterology : WJG. 2012;18:616-26.

37. Labus JS, Bolus R, Chang L, Wiklund I, Naesdal J, Mayer EA, Naliboff BD. The Visceral Sensitivity Index: development and validation of a gastrointestinal symptom-specific anxiety scale. Aliment Pharmacol Ther. 2004;20:89-97.

38. Jerndal P, Ringström G, Agerforz P, Karpefors M, Akkermans LM, Bayati A, Simrén M. Gastrointestinalspecific anxiety: an important factor for severity of $\mathrm{Gl}$ symptoms and quality of life in IBS. Neurogastroenterol Motil. 2010;22:646-e179.

39. Ruepert L, Quartero AO, de Wit NJ, van der Heijden GJ, Rubin G, Muris JW. Bulking agents, antispasmodics and antidepressants for the treatment of irritable bowel syndrome. The Cochrane database of systematic reviews 2011: CD003460.

40. Sabate JM VM, Mion F, Siproudhis L, Ducrotte P, Zerbib F, Grimaud JC, Dapoigny M, Dyard F, Coffin B. Relationship between rectal sensitivity, symptoms intensity and quality of life in patients with irritable bowel syndrome. Aliment Pharmacol Ther. 2008;28:484-90. 


\section{Chapter}

Small intestinal permeability is increased in diarrhoea predominant IBS, while alterations in gastroduodenal permeability in all IBS subtypes are largely attributable to confounders

Z Mujagic*, S Ludidi*, D Keszthelyi, MAM Hesselink, JW Kruimel, K Lenaerts, NMJ Hanssen, JM Conchillo, DMAE Jonkers, AAM Masclee

* Both authors contributed equally to the manuscrip Aliment Pharmacol and Ther - Epub ahead of print 


\section{Abstract}

\section{Background}

Intestinal permeability has been studied in small groups of IBS patients with contrasting findings.

Aim

The aims of the present study were to assess intestinal permeability at different sites of the GI tract in different subtypes of well-characterised IBS patients and healthy controls (HC) and to assess potential confounding factors.

\section{Methods}

IBS patients and HC underwent a multi-sugar test to assess site-specific intestinal permeability. Sucrose excretion and lactulose/rhamnose ratio in $0-5 \mathrm{~h}$ urine indicated gastroduodenal and small intestinal permeability, respectively. Sucralose/erythritol ratio in 0-24 h and 5-24 h urine indicated whole gut and colonic permeability, respectively. Linear regression analysis was used to assess the association between IBS groups and intestinal permeability and to adjust for age, sex, $\mathrm{BMI}$, anxiety or depression, smoking, alcohol intake, and use of medication.

\section{Results}

91 IBS patients, i.e. $37 \%$ IBS-D, 23\% IBS-C, 33\% IBS-M and 7\% IBS-U, and $94 \mathrm{HC}$ were enrolled. Urinary sucrose excretion was significantly increased in the total IBS group ( $\mu$ mol, median [Q1;Q3]: 5.26 [1.82;11.03] vs. 2.44 [0.91;5.85], P<0.05), as well as in IBS-C and IBS-D vs. HC. However, differences attenuated when adjusting for confounders. The lactulose/rhamnose ratio was increased in IBS-D vs. HC $(0.023[0.013 ; 0.038]$ vs. $0.014[0.008 ; 0.025], P<0.05)$, which remained significant after adjustment for confounders. No difference was found in 0-24 and 5-24 hour sucralose/erythritol ratio between groups.

\section{Conclusion}

Small intestinal permeability is increased in patients with IBS-D compared to $\mathrm{HC}$, irrespective of confounding factors. Adjustment for confounders is necessary when studying intestinal permeability, especially in a heterogeneous disorder such as IBS. 


\section{Introduction}

Irritable bowel syndrome (IBS) is a frequently occurring functional disorder of the gastrointestinal (GI) tract, characterised by abdominal pain and alterations in bowel habits. It is associated with impaired quality of life, increased use of health care services and high health care costs $^{1,2}$. The IBS patient population is phenotypically very heterogeneous, with a diversity of predominant symptoms and clinical characteristics ${ }^{3}$, reflecting the multitude of underlying pathophysiological mechanisms ${ }^{4}$.

Changes in gut epithelial barrier function have been observed in IBS and have been postulated to play a role in the pathogenesis of the disorder. Several studies have pointed to an increased intestinal permeability in IBS patients when compared to healthy controls. However, these findings were based on data obtained in small study populations $^{5-7}$, or only in specific IBS subpopulations, such as post-infectious IBS cohorts $^{7-9}$ or diarrhoea predominant IBS (IBS-D) $)^{5,10,11}$. Alterations in intestinal permeability appear to be subtype-related and site-specific, as several studies found an increased small intestinal permeability in patients with IBS- $D^{5,7,10,11}$. Data on colonic and whole gut permeability are inconsistent ${ }^{5-7,12,13}$ and only a limited number of studies investigated a non-selected heterogeneous IBS population ${ }^{6,12,14}$ or focussed also on patients with constipation predominant IBS (IBS-C) ${ }^{7,13}$. Comparison of data from published studies is further hampered by differences in methodology of permeability tests. Furthermore, factors such as demographical characteristics, medication and lifestyle are known to influence intestinal permeability, but currently available information on possible confounding effects by these factors on intestinal permeability in IBS patients is very limited ${ }^{8,12}$.

Therefore, data are needed from larger well-characterised IBS cohorts using validated site-specific intestinal permeability measurements for the different anatomical GI locations, comparing different subtypes and taking possible confounders into account.

We hypothesized that 1) IBS patients have increased intestinal permeability compared to healthy controls ( $\mathrm{HC})$, which is site-specific and subtype-dependent and 2) intestinal permeability in IBS patients is affected by factors such as demographical or lifestyle characteristics, psychological symptoms and use of medication.

Aims of the present study were 1) to assess intestinal permeability of different sites of the GI tract, in a large group of well-characterised IBS patients and healthy controls and to investigate whether differences exist between IBS subtypes and 2) to assess potential confounding effects of demographical characteristics, psychological and lifestyle factors and use of medication on intestinal permeability. 


\section{Materials and methods}

\section{Study participants}

The present study is part of a larger cohort study on the phenotypical and genotypical characterisation of patients with IBS, with age and gender matched healthy controls, wherein multiple parameters were assessed according to standardised procedures. The subgroup of patients and controls that underwent an intestinal permeability test was included in the current study.

Between September 2009 and June 2013, subjects between 18 and 75 years of age, with IBS diagnosed by the Rome III criteria were recruited via the outpatient department of the division Gastroenterology-Hepatology of the Maastricht University Medical Centert, a secondary and tertiary referral centre. Medical history was taken by a gastroenterologist. GI endoscopy with biopsies, abdominal imaging by ultrasonography or CT scan and/or blood, breath and faecal analysis were performed to exclude organic disease, if indicated. Patients with a history of abdominal surgery, except appendectomy, laparoscopic cholecystectomy and hysterectomy, and patients who were not able to abide the instructions for the permeability test were excluded. IBS patients were assigned to subtypes, i.e. diarrhoea (IBS-D), constipation (IBS-C), mixed stool pattern (IBS-M) and unspecified stool pattern (IBS-U), based on predominant bowel habits according to the ROME III criteria and daily Bristol Stool Chart in a 14-day symptom dairy ${ }^{15}$.

Healthy subjects in the same age category were included, as controls (HC). They were enrolled via public advertising on websites and in local newspapers. Before participation, a brief medical history was taken to exclude the presence of GI disorders and current or previous GI complaints. All measurements performed in IBS patients were also carried out in $\mathrm{HC}$.

All subjects have given written informed consent prior to their inclusion. The study protocol has been approved by the Maastricht University Medical Center+ Committee of Ethics and was executed according to the revised Declaration of Helsinki $\left(59^{\text {th }}\right.$ general assembly of the WMA, Seoul, South Korea, Oct. 2008). The study has been registered in the US National Library of Medicine (http://www.clinicaltrials.gov, NCT00775060).

\section{Permeability measurement}

Intestinal permeability was assessed on four sites of the GI tract. Sucrose excretion and the lactulose and rhamnose (L/R) ratio in 0-5 hour urine were used as indicators of gastroduodenal and small intestinal permeability, respectively. Whole-gut permeability was indicated by the sucralose and erythritol (S/E) ratio in 0-24 hour urine, whereas the 5-24 hour S/E ratio was used as indicator for colonic permeability (Figure 5.1) ${ }^{16-18}$. 
All patients and $\mathrm{HC}$ included in this study underwent a non-invasive validated multisugar test, measuring urinary excretion of ingested sugar probes indicating gastroduodenal, small intestinal, colonic and whole gut permeability, as previously validated by van Wijck et al. ${ }^{17,18}$ During and two days prior to the test day excessive physical exercise, consumption of alcohol and intake of non-steroidal anti-inflammatory drugs (NSAIDs) were prohibited, to prevent short term effects of these factors on intestinal permeability.

After an overnight fast, participants emptied their urinary bladder and consumed a multi-sugar drink (150 $\mathrm{ml}$ of tap water) containing $1 \mathrm{~g}$ sucrose (van Gilse, Dinteloord, the Netherlands), $1 \mathrm{~g}$ lactulose (Centrafarm, Etten-Leur, the Netherlands), $0.5 \mathrm{~g}$ L-rhamnose (Danisco, Copenhagen, Denmark), 1 g sucralose (Brenntag, Sittard, the Netherlands) and $1 \mathrm{~g}$ erythritol (Now Foods, Bloomindale, USA). Patients were requested to collect 24 hour urine in two separate fractions, i.e. 0-5 and 5-24 hour fraction. During the first 5 hours of urine collection, neither foods nor drinks were allowed with the exception of water. Total urine volume was determined and urine samples were aliquoted and frozen at $-80^{\circ} \mathrm{C}$ until analyses of the sugar probes by HPLC-MS as previously described ${ }^{17,18}$.

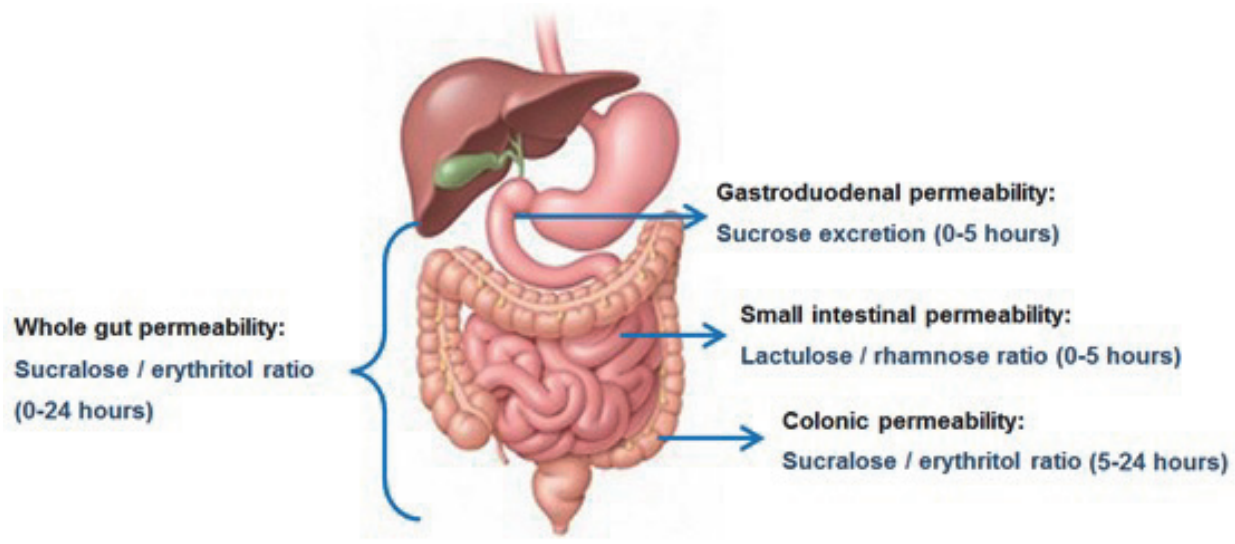

Figure 5.1 Multi-sugar test, indicating permeability of four sites of the Gl tract.

\section{Questionnaires}

Within a period of two weeks prior to the permeability measurement, questionnaires on demographical characteristics, lifestyle factors and the use of medication were completed by the participants. The presence of GI symptoms was assessed using the validated gastrointestinal symptom rating scale (GSRS), consisting of 16 items clustered into five major GI syndromes: abdominal pain, reflux, diarrhea, indigestion and 
constipation syndrome ${ }^{19}$. Moreover, symptoms of discomfort, abdominal pain, bloating, flatulence, nausea and intensity of total complaints were scored daily during 14 days, on a 5-point Likert-scale, in a symptom diary. Subsequently, the mean symptom composite score (MSS) was calculated, based on sum of individual symptoms per day ${ }^{20}$.

Symptoms of anxiety and depression were assessed using the hospital anxiety and depression scale (HADS). This questionnaire consists of two 7-item scales, that is one for anxiety and one for depression, each with a Likert scoring range between $0-3$. A higher score indicates a higher degree of distress and a total score $\geq 8$ on either the anxiety or depression scale is indicative for the presence of anxiety or depression symptoms, respectively ${ }^{21,22}$.

\section{Data and statistical analysis}

Statistical analysis was performed using IBM SPSS Statistics version 21.0 (IBM Statistics for Macintosh, Chicago IL).

The total IBS group is referred to as IBS TOTAL. Data of IBS TOTAL $_{\text {TA }}$ and IBS subtypes versus HC were compared using linear regression analysis. Due to skewed distribution, urinary sugar excretions and ratios were natural logarithmic (LN) transformed. Linear regression analysis was performed to assess the association between presence of IBS and intestinal permeability (Model 1). Furthermore, we investigated possible confounding effects of disease-related factors on intestinal permeability. Therefore, we first adjusted the crude analysis for demographical characteristics (age, sex, BMI), (Model 2). Next, we additionally adjusted for psychological (anxiety or depressive) symptoms, (Model 3). We then additionally adjusted for lifestyle factors (smoking history, defined as current or previous smoker, and high use of alcohol, defined as more than 15 units per week), (Model 4), and thereafter additionally for use of medication in the two weeks prior to inclusion (NSAID, proton pump inhibitors (PPI), selective serotonin reuptake inhibitors (SSRI) and medication that affects motility, i.e. laxatives, prokinetics, opioids, spasmolytics and anti-diarrhoeal drugs), (Model 5). All analyses were performed for IBS TOTAL $_{\text {as }}$ well as for the subtypes in a separate analysis as dummy variables. To investigate which covariate had the strongest confounding effect on the association between presence of IBS and sugar excretion or ratios, we additionally adjusted the crude analysis (Model 1) for all factors separately. The factors with the strongest significant effects where thereafter omitted separately from the fully adjusted model (Model 1-5) to determine if the factors are indeed confounders and affect the association between IBS and sugar excretion or ratios or if the factors are independently of the presence of IBS associated with sugar excretion or ratios.

Missing values were excluded listwise. A two-sided $p$-value of 0.05 was considered statistically significant. The $ß$ of the linear regression analyses signifies one $L N$ change in sugar excretion and ratios versus $\mathrm{HC}$. 
Finally, Spearman's rank was used to assess correlation between urinary sugar excretions or ratios and GI symptoms.

\section{Results}

\section{Demographical characteristics}

Intestinal permeability data were available from 91 IBS patients and $94 \mathrm{HC}$. Predominant bowel habits for diarrhoea (IBS-D), constipation (IBS-C), mixed stool pattern (IBS-M) and unspecified stool pattern (IBS-U) were seen in $37 \%(n=34), 23 \%$ $(n=21), 33 \%(n=30)$ and $7 \%(n=6)$ of the patients, respectively. Due to the small number of patients with IBS-U, this group has not been analysed as a separate subtype. All patients were included in the analysis of the group IBS TOTAL.

Demographical characteristics, GI and psychological symptoms, lifestyle parameters

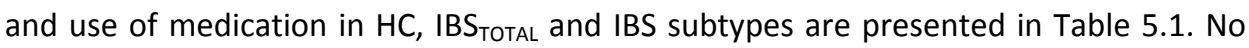
statistically significant differences were observed for age, sex and BMI between groups. Symptoms of anxiety and depression, as well as use medication were significantly higher in IBS TOTAL versus $\mathrm{HC}$. Overall alcohol consumption was significantly lower in IBSTOTAL when compared to HC, and this was also true for high alcohol intake, i.e. above 15 units per week. As to be expected, severity of all $\mathrm{Gl}$ symptoms was significantly higher in IBS patients versus $\mathrm{HC}$ and bowel habits were altered according to IBS subtype.

\section{Urinary sugar excretion}

Data of absolute recovery of urinary excreted sugars per urine fraction is provided in Supplementary Table S5.I.

\section{Gastroduodenal permeability}

In Table 5.2, median and ranges of urinary sucrose excretion (in 0-5h urine fraction) are given for HC, IBS TOTAL, IBS-D, IBS-C and IBS-M. Linear regression analysis of sucrose excretion with adjustments for possible confounders is provided as Supplementary Table S5.2. 
Table 5.1 Demographical characteristics, psychological symptoms, lifestyle parameters, medication use and $\mathrm{GI}$ symptoms in healthy controls (HC), IBS patients (IBS TOTAL $_{\text {) }}$ and IBS subtypes (i.e. IBS-D, IBS-C and IBS-M).

\begin{tabular}{|c|c|c|c|c|c|}
\hline Parameter & $\begin{array}{c}\mathrm{HC} \\
(\mathrm{N}=94)\end{array}$ & $\begin{array}{l}\mathrm{IBS}_{\text {TOTAL }} \\
(\mathrm{N}=91)\end{array}$ & $\begin{array}{c}\text { IBS-D } \\
(\mathrm{N}=34)\end{array}$ & $\begin{array}{c}\text { IBS-C } \\
(N=21)\end{array}$ & $\begin{array}{l}\text { IBS-M } \\
(\mathrm{N}=30)\end{array}$ \\
\hline \multicolumn{6}{|l|}{ Demographical characteristics } \\
\hline Age (mean years \pm SEM) & $45.0 \pm 1.95$ & $44.4 \pm 1.61$ & $45.0 \pm 2.40$ & $47.5 \pm 3.57$ & $42.23 \pm 3.06$ \\
\hline Female sex $(\%)$ & 58.5 & 63.7 & 57.1 & 61.9 & 70 \\
\hline $\mathrm{BMI}\left(\mathrm{kg} / \mathrm{m}^{2}\right)$ & $23.8 \pm 0.48$ & $25.2 \pm 0.56$ & $25.6 \pm 1.23$ & $25.0 \pm 0.91$ & $25.4 \pm 0.69$ \\
\hline \multicolumn{6}{|l|}{$\begin{array}{l}\text { Anxiety and depressive symptoms } \\
\text { (HADS } \geq 8)\end{array}$} \\
\hline Depressive symptoms (\%) & 7.7 & $20.5^{a}$ & $28.6^{a}$ & 20.0 & 17.1 \\
\hline Anxiety symptoms (\%) & 12.1 & $39.8^{c}$ & $45.7^{c}$ & $35.0^{\mathrm{a}}$ & $35.7^{a}$ \\
\hline \multicolumn{6}{|l|}{ Lifestyle parameters } \\
\hline Positive smoking history (\%) & 42.6 & 51.6 & $62.9^{a}$ & 57.1 & 36.7 \\
\hline Alcohol intake 0 units/week (\%) & 16.3 & $40.9 * * *$ & $40.0 * *$ & 30.0 & $46.4 * *$ \\
\hline Alcohol intake >15 units/week (\%) & 11.7 & $1.1^{\mathrm{b}}$ & 2.9 & 0.0 & $0.0^{\mathrm{a}}$ \\
\hline \multicolumn{6}{|l|}{$\begin{array}{l}\text { Use of medication } 2 \text { weeks prior to } \\
\text { participation }\end{array}$} \\
\hline PPI (\%) & 2.1 & $28.6^{c}$ & $31.4^{c}$ & $23.8^{\mathrm{b}}$ & $33.3^{c}$ \\
\hline NSAID (\%) & 5.3 & $14.3^{\mathrm{a}}$ & 8.6 & 19.0 & 16.7 \\
\hline SSRI (\%) & 1.1 & $15.4^{c}$ & $17.1^{b}$ & 0.0 & $23.3^{c}$ \\
\hline Motility + drugs (\%) & 0.0 & $19.8^{c}$ & $14.3^{a}$ & $23.8^{c}$ & $26.7^{c}$ \\
\hline Motility - drugs (\%) & 0.0 & $12.1^{c}$ & $17.1^{c}$ & 9.5 & $10.0^{*}$ \\
\hline Statins (\%) & 5.3 & 8.8 & 5.7 & 14.3 & 6.7 \\
\hline Antihypertensive med. (\%) & 11.7 & 16.5 & 14.3 & 23.8 & 16.7 \\
\hline \multicolumn{6}{|l|}{ GSRS syndrome (Mean \pm SEM) } \\
\hline Abdominal pain & $1.60 \pm 0.07$ & $3.20 \pm 0.12^{c}$ & $3.28 \pm 0.19^{c}$ & $3.30 \pm 0.26^{c}$ & $3.18 \pm 0.21^{\mathrm{c}}$ \\
\hline Reflux syndrome & $1.18 \pm 0.05$ & $2.22 \pm 0.16^{c}$ & $2.30 \pm 0.26^{c}$ & $2.15 \pm 0.34^{c}$ & $2.29 \pm 0.29^{c}$ \\
\hline Diarrhoea syndrome & $1.41 \pm 0.07$ & $3.55 \pm 0.15^{c}$ & $4.03 \pm 0.19^{c}$ & $2.76 \pm 0.33^{c}$ & $3.7 \pm 0.27^{c}$ \\
\hline Indigestion syndrome & $1.97 \pm 0.09$ & $4.04 \pm 0.13^{c}$ & $4.21 \pm 0.23^{c}$ & $3.94 \pm 0.27^{c}$ & $4.16 \pm 0.19^{c}$ \\
\hline Constipation syndrome & $1.61 \pm 0.09$ & $3.35 \pm 0.15^{c}$ & $2.70 \pm 0.231^{c}$ & $3.86 \pm 0.40^{c}$ & $3.84 \pm 0.18^{c}$ \\
\hline \multicolumn{6}{|l|}{$\begin{array}{l}\text { Symptom scores - } 14 \text { day average } \\
\text { (Mean } \pm \text { SEM) }\end{array}$} \\
\hline Discomfort & $1.12 \pm 0.02$ & $2.45 \pm 0.09^{c}$ & $2.39 \pm 0.14^{c}$ & $2.52 \pm 0.18^{c}$ & $2.46 \pm 0.18^{c}$ \\
\hline Abdominal pain & $1.06 \pm 0.02$ & $2.29 \pm 0.10^{c}$ & $2.16 \pm 0.14^{c}$ & $2.45 \pm 0.20^{c}$ & $2.24 \pm 0.18^{c}$ \\
\hline Bloating & $1.10 \pm 0.02$ & $2.21 \pm 0.12^{c}$ & $2.15 \pm 0.19^{c}$ & $2.20 \pm 0.21^{c}$ & $2.40 \pm 0.26^{c}$ \\
\hline Flatulence & $1.33 \pm 0.04$ & $2.44 \pm 0.11^{c}$ & $2.64 \pm 0.20^{c}$ & $2.13 \pm 0.13^{c}$ & $2.51 \pm 0.21^{c}$ \\
\hline Intensity of symptoms & $1.10 \pm 0.02$ & $2.49 \pm 0.10^{c}$ & $2.38 \pm 0.14^{c}$ & $2.51 \pm 0.20^{c}$ & $2.54 \pm 0.20^{c}$ \\
\hline Mean symptom composite score & $6.73 \pm 0.09$ & $13.33 \pm 0.49^{c}$ & $13.18 \pm 0.80^{c}$ & $13.35 \pm 0.91^{c}$ & $13.60 \pm 0.97^{c}$ \\
\hline
\end{tabular}

Motility + drugs: medication that positively affect motility, i.e. laxatives and prokinetic drugs; motility - drugs: medication that negatively affect motility, i.e. opioids, spasmolytics and anti-diarrhoeal drugs. GSRS represents symptom scores for abdominal pain, reflux, diarrhoea, indigestion and constipation syndrome. End-of-day symptom diary is showed as day average over 14 days. A mean symptom composite score (MSS) was calculated, based on sum of individual symptoms in the diary per day.

Difference between IBS TOTAL, IBS-D, IBS-C and IBS-M compared to HC tested with linear regression. ${ }^{\mathrm{a}} P<0.05$; ${ }^{\mathrm{b}} P<0.01 ;{ }^{\mathrm{c}} P<0.001$ vs. HC 
Crude analysis showed a significantly higher sucrose excretion in IBS TOTAL $_{\text {(median [Q1; }}$ Q3] in $\mu$ mol: 5.26 [1.82; 11.03] $P=0.007)$, IBS-D $(4.22$ [2.12; 8.03] $P=0.049)$ and IBS-C (7.40 [2.37; 18.29] $P=0.004)$ compared to HC (2.44 [0.91; 5.85]). After adjustment for sex, age and BMI, sucrose excretion remained significantly higher in IBS TOTAL $_{\text {Ta }}$ relative to $\mathrm{HC},(\beta: 0.49,95 \% \mathrm{Cl}: 0.05 ; 0.94, P=0.031)$. This association was no longer significant when we additionally adjusted for psychological symptoms $(\beta: 0.44,95 \% \mathrm{Cl}:-0.04 ; 0.91$, $P=0.073$ ), for lifestyle factors ( $\beta$ : $0.37,95 \% \mathrm{Cl}:-0.12 ; 0.86, P=0.133$ ) and almost completely attenuated after additional adjustment for use of medication ( $\beta$ : $-0.04,95 \%$ $\mathrm{Cl}:-0.59 ; 0.51, P=0.880)$. Overall, we found similar results for the IBS subtypes relative to HC. Although associations appeared to be stronger within the IBS-C group, these were no longer significant after adjustment for medication use $(\beta: 0.41,95 \% \mathrm{Cl}:-0.39$; 1.20, $P=0.312$ ).

When separately adjusting the crude analysis for the possible confounders, several factors were associated with higher sucrose excretion. A higher $\mathrm{BMI}(\beta: 0.07,95 \% \mathrm{Cl}$ : $0.02 ; 0.11, P=0.004)$, as well as a positive smoking history $(\beta: 0.46,95 \% \mathrm{Cl}: 0.02 ; 0.89$, $P=0.040)$, were related to higher sucrose excretion. Male sex $(\beta: 0.43,95 \% \mathrm{Cl}:-0.01$; $0.87, P=0.056)$ and use of PPI's ( $\beta: 0.59,95 \% \mathrm{Cl}:-0.08 ; 1.25, P=0.084)$ were associated with higher sucrose excretion as well, but did not reach statistical significance. Drugs that positively affect motility, i.e. laxatives and prokinetics, showed the strongest association with sucrose excretion ( $\beta$ : $0.77,95 \% \mathrm{Cl}$ : 0.02; 0.152, $P=0.045$ ).

Thereafter we omitted separately BMI, smoking, sex, use of PPI, laxatives and prokinetics from the fully adjusted model. When omitting BMI and the use of laxatives and prokinetics the association between sucrose excretion with IBS TOTAL $_{\text {Ta }}$ as well as with the IBS subtypes became stronger again, demonstrating the confounding effect of these factors in our population, while the other three factors did not affect the analysis (data not shown) and are therefore not confounders in this analysis but are independently associated with increased sucrose excretion.

\section{Small intestinal permeability}

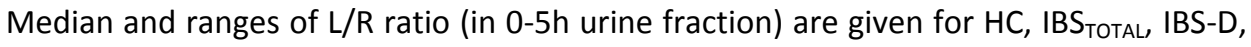
IBS-C and IBS-M in Table 5.2. Linear regression analysis of $L / R$ ratio with adjustments for possible confounders is provided as Supplementary Table S5.3.

Crude analysis showed a significantly higher L/R ratio in IBS-D (median [Q1; Q3]: 0.023 [0.013;0.038], $P=0.029)$ compared to $\mathrm{HC}(0.014$ [0.008; 0.025]), but no significant

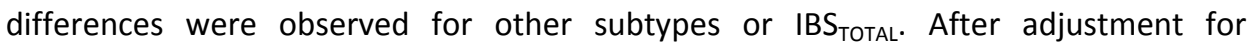
demographical factors, psychological symptoms and lifestyle, the $L / R$ ratio remained significantly higher for IBS-D relative to $\mathrm{HC}(\beta: 0.53,95 \% \mathrm{Cl}: 0.03 ; 1.03, P=0.037)$. This association was even slightly stronger when we additionally adjusted for use of medication ( $\beta: 0.63,95 \% \mathrm{Cl}$ : 0.09; 1.16, $P=0.022$ ). 

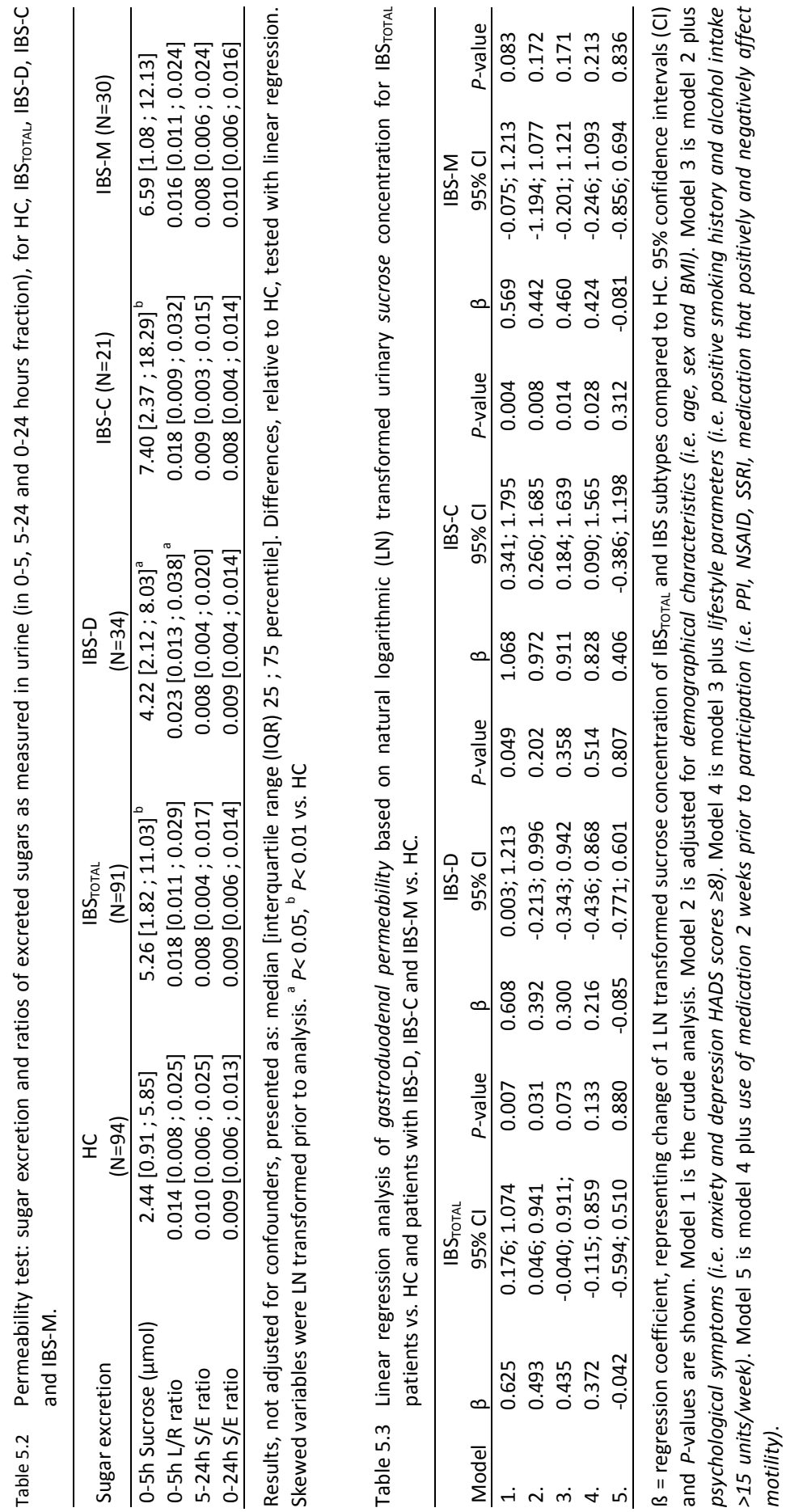


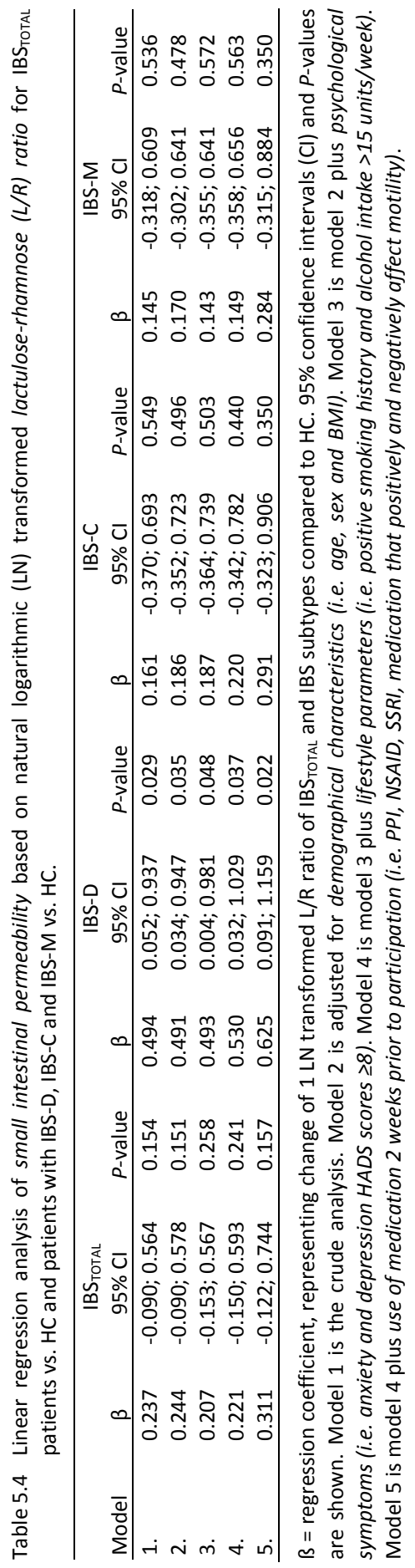


Separately adjusting the crude analysis for each factor revealed that only male sex was associated with increased L/R ratio $(\beta: 0.31,95 \% \mathrm{Cl}:-0.01 ; 0.63, P=0.054)$, although not reaching statistical significance. When thereafter adjusting the crude analysis for all factors combined and excluding sex, the associations between IBS groups and L/R ratio was not affected (data not shown), demonstrating that the effect of the investigated confounders on the association between IBS and L/R ratio is small.

\section{Colon and whole gut permeability}

Linear regression analysis of S/E-ratio in the 5-24 $\mathrm{h}$ of urine fraction, indicating colonic permeability, and in 0-24 h urine, indicating whole-gut permeability, without (see also Table 5.2) or with adjustments for possible confounding factors (data not shown), did

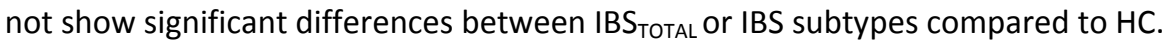

\section{Correlation between intestinal permeability and symptoms}

Symptoms of abdominal pain, diarrhea and indigestion syndrome as measured by the GSRS, showed a statistically significant but weak positive correlation with sucrose excretion (all Spearman's rho<0.27; $P=0.001$ ). Diarrhea and indigestion syndrome also showed a significant but weak positive correlation with L/R ratio (all Spearman's rho<0.17; $P<0.05)$. No significant correlation was found for other symptoms, nor between GI symptoms and colonic or whole gut permeability. Correlations between symptoms measured by the 14-days symptom diary and intestinal permeability were comparable (data not shown).

\section{Discussion}

In this paper, we report on gut permeability testing, employing a validated multi-sugar test that simultaneously measures permeability at various sites in the GI tract, in a large and well-characterized IBS population and a healthy control group. In addition, we evaluated the effect of possible confounding factors. Sucrose excretion was significantly higher in IBS TOTAL, IBS-D and IBS-C when compared to controls and the L/R ratio was significantly higher in IBS-D patients. However, after adjustment for possible confounders, only the L/R ratio, indicating small intestinal permeability, in IBS-D remained significantly increased when compared to controls.

We have used a validated multi-sugar test to analyse site-specific permeability of the intestine. The advantage of this test is that low dosages of 5 different sugars were given and analysed in two urine fractions, i.e. $0-5 \mathrm{~h}$ and 5-24h, as indicators for gastroduodenal, small intestinal, colonic and whole gut permeability (see also Figure 
5.1). The site-specificity of the different sugars and ratios used is tested previously in animal and human studies ${ }^{16-18,23-25}$. Sucrose (a disaccharide) is hydrolysed by the enzyme sucrase in the duodenum, ${ }^{23}$ and has been demonstrated to indicate gastroduodenal permeability when measured in 0-5 h urine. Lactulose (a disaccharide, not broken down by human enzymes) can permeate by the paracellar route and rhamnose (a mono-saccharide) by the transcellular route. As both are degraded by bacteria that are most abundant in the colon, ${ }^{16}$ they are typically used as markers for small intestinal permeability ${ }^{16-18,24,26,27}$. With respect to urine sampling after sugar intake, it is not yet clear whether $0-1 \mathrm{~h}, 0-2 \mathrm{~h}$ or $0-5 \mathrm{~h}$ urine fractions best reflect small intestinal permeability. Data from van Wijck et al. indicate however, that $0-5 \mathrm{~h}$ fractions in the applied multi-sugar test, do reflect small intestinal permeability ${ }^{17,18}$. Sucralose (a disaccharide) and erythritol (a sugar alcohol) are both not broken down by human enzymes nor by human colonic bacteria ${ }^{28,29}$. Therefore ratios of these sugars in 5-24 $\mathrm{h}$ and $0-24 \mathrm{~h}$ urine are used as indicators for colonic and whole gut permeability, respectively ${ }^{16-18,24}$. Permeability testing by sugar probes could possibly be affected by differences in gastric emptying and intestinal transit time, which were not assessed in our study. However, by using a total ingested sugar amount of only $4.5 \mathrm{~g}$ and the ratios of transcellularly and paracellularly absorbed sugar probes, these effects were minimized.

We found a significant difference in sucrose excretion, pointing to increased gastroduodenal permeability in IBS TOTAL $_{\text {Ts }}$ well as in IBS-C and IBS-D patients compared to HC. However, after adjusting for patient characteristics and psychological symptoms,

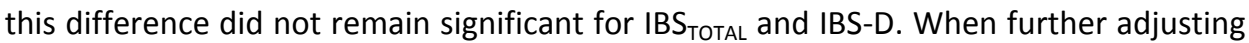
for lifestyle factors and use of medication the difference did not remain significant for IBS-C either, indicating that the observed difference was mainly based on confounding factors in our population.

Although IBS is a heterogeneous disease associated with a wide variety of patient characteristics and environmental factors (e.g. sex differences, psychosocial events, medication), most studies assessing intestinal permeability in IBS patients have not taken into account the possible confounding effects of these factors. Therefore it is not known whether their observations on permeability were associated with the disease per se or with the presence of possible confounders. Only two studies reported on a limited number of possible confounders. In the Walkerton cohort of post infectious IBS patients, male sex was found to be associated with increased lactulose-mannitol ratio in overnight urine fractions, ${ }^{8}$ which was previously demonstrated in healthy controls, ${ }^{30}$ pointing to a physiological sex difference. Furthermore NSAIDs were shown to compromise whole gut permeability in both IBS patients and healthy controls. ${ }^{12}$ 
We have analysed a large number of potential confounding factors. A higher BMI was shown to have an important confounding effect on the association between IBS and sucrose excretion in our study, which is in line with previously reported increased intestinal permeability in obese healthy subjects compared to lean ${ }^{31}$. The most pronounced effect on sucrose excretion was found when we separately adjusted for the use of drugs that positively affect motility, i.e. prokinetics and laxatives. A possible mechanism by which prokinetics increase intestinal permeability could be the dopaminergic receptor stimulation by these drugs which in animal models was found to be associated with intestinal barrier alterations ${ }^{32}$. One study postulated that laxatives increase intestinal permeability by direct effects on tight junction proteins ${ }^{33}$.

Male sex, smoking and use of PPI's were associated with higher sucrose excretion in our study, but did not have a confounding effect in our study population. In previous studies an increased sucrose excretion was found in patients with gastro-oesophageal reflux disease as well as in healthy controls that use PPI's. It was hypothesized that this may be caused by PPI-related effects on the intracellular potassium and calcium homeostasis, which could affect the cytoskeleton and thereby tight junctions between epithelial cells ${ }^{34}$. We cannot fully rule out that PPIs or other medications did not have any causal effect on permeability, but rather that the underlying condition for which they were used were the cause of the observed association. However, we did not see differences in permeability on all GI sites between patients with and without reflux/dyspepsia symptoms, being conditions clearly associated with PPI use. With respect to smoking, previous reports showed no difference in intestinal permeability between smoking and non-smoking patients with inflammatory bowel disease and healthy subjects ${ }^{35,36}$. With respect to use of NSAIDs, it has been observed that these drugs can increase intestinal permeability in IBS patients and healthy subjects shortly after intake, i.e. acute effects ${ }^{12,27}$. We instructed study subjects to refrain from use of NSAIDs or alcohol in the two days prior and during the permeability test, in order to eliminate acute effect of these substances on intestinal permeability. Therefore, our data reflect chronic rather than acute effects and these factors did not reveal to be confounders in our population. In a post-hoc analysis we also tested the effect of alcohol intake versus no alcohol intake in general for our population, and no effect of alcohol intake on intestinal permeability was observed in this case as well (data not shown).

The urinary $L / R$ ratio was affected and points to an increased small intestinal permeability in patients with IBS-D compared to HC, but not in other IBS-subtypes. Our finding is in line with previous observations in small sized studies (12 to 19 IBS-D patients) $)^{5,7,11}$. The significant difference in L/R ratio between IBS-D and HC that we observed persisted after adjustment for confounders. 
The increased permeability, reflecting intestinal barrier dysfunction of the small bowel, may represent an important pathophysiological mechanism in IBS-D. This finding is supported by recent mechanistic studies revealing not only an increased number and activation of mast cells in jejunal biopsies of IBS-D patients compared to HC, but also significant differences in global gene expression, particularly in tight-junction signaling pathways, $^{37}$ and structural alterations of the apical junction complex correlating positively with mast cell activation as well as with clinical symptoms ${ }^{38}$. These data support the concept of IBS-D as a functional gut disorder or entity having specific organic substrates and underlying pathophysiological mechanisms.

Whether increased intestinal permeability is an early event or consequence of IBS remains to be determined. Previous findings indicate that barrier dysfunction may contribute to symptom generation in $\mathrm{IBS}^{10,38,39}$ and point to the direction of barrier dysfunction as an early event, facilitating permeation of e.g. bacteria and their products (i.e. lipopolysaccharides), resulting in immune activation ${ }^{40,41}$ which may further increase paracellular epithelial permeability and subsequent low-grade mucosal inflammation ${ }^{42-44}$. Increased intestinal permeability has also been associated with visceral hypersensitivity in IBS patients ${ }^{10}$. However, it has to be noted that increased intestinal permeability may be a consequence, rather than a causative factor of IBS. Recent studies have shown that acute stress and factors along the gut brain axis, e.g. serotonin $^{45}$ and corticotropin-releasing factor (CRF), ${ }^{46}$ modulate intestinal permeability by mast cell-dependent mechanisms in murine models as well as in healthy human subjects, postulating that the enteric and central nervous system may have a regulatory effect on the intestinal barrier function ${ }^{46-49}$. Since this evidence exists for an association between acute stress and increased intestinal permeability in healthy individuals, we have analysed the influence of chronic symptoms of anxiety and depression on intestinal permeability (Model 2) in IBS patients. Symptoms of anxiety and depression appear to account for part of the increased sucrose excretion and L/R ratio in IBS patients; however, in the additional analysis of the separate co-factors the effect of psychological symptoms on intestinal permeability was only weak and not statistically significant.

The identification of increased small intestinal permeability in patients with IBS-D may become relevant from a therapeutic perspective. However, first more mechanistic research is needed to provide a definition of a clinically relevant difference in intestinal permeability, e.g. associated to increased bacterial translocation or endotoxin levels and subsequent mucosal inflammation contributing to symptom generation in the target population. 
Previous data on in vivo colonic permeability in IBS or IBS-D are contrasting, i.e. lower, ${ }^{6}$ higher $^{5,11,13}$ or unaltered colonic permeability ${ }^{7}$ when compared to $\mathrm{HC}$. There are however some indications of impaired barrier function in studies focussing on tight junctions in colonic biopsies from IBS patients, with reduced expression of zonula occludens-1 and occludin proteins ${ }^{50}$. Previous reports on whole gut permeability do point to the direction of increased permeability in post-infectious IBS and IBS-D, ${ }^{8,10}$ while no differences were found for IBS patients in general when compared to $\mathrm{HC}^{6,12}$. However, all studies used different methods to assess the colonic and whole gut permeability and included relatively small numbers of subjects or assessed permeability only in post-infectious IBS patients. Our aim was to clarify this point by assessing colonic as well as whole gut permeability in a large group of IBS patients, using the validated multi-sugar test. Our results however, do not show statistically significant differences in S/E ratio in 5-24 $\mathrm{h}$ and 0-24 $\mathrm{h}$ urine fraction, indicating no alternations in colonic or whole gut permeability, respectively, in IBS TOTAL or IBS subtypes when compared to $\mathrm{HC}$.

IBS is a very heterogeneous disorder that is associated with a multitude of patient characteristics and chronic or intermittent use of medication. Based on literature data, we anticipated that some of these factors would affect intestinal permeability. Our results demonstrate that especially sucrose excretion was affected by confounders, thereby altering the conclusion with respect to gastroduodenal permeability in IBS patients. Based on the present findings, especially BMI, smoking history, medication and sex should be taken into account as possible confounders in future permeability studies. Moreover, after adjustment of our analysis for possible confounding factors only small intestinal permeability (L/R ratio) in IBS-D patients remained significantly increased compared to HC.

Our findings clearly show the relevance of adjustment for possible confounding factors, as well as site-specific permeability assessment and IBS subtype differentiation in future studies analysing intestinal permeability in IBS patients.

Although we assessed intestinal permeability in a large heterogeneous IBS population, and the range of our confidence intervals of our fully adjusted models were similar to the crude model, and while analyses in our IBS subpopulations were largely consistent with our IBS TOTAL $_{\text {Tal }}$ analyses, a study including a larger number of patients in separate IBS subtype groups would be warranted to confirm our findings in the subgroup analyses. Furthermore, it has to be noted that our results are based on cross-sectional and observational data and that causality was not studied. 


\section{Conclusion}

By assessing intestinal permeability in a large group of IBS patients and healthy controls, we have demonstrated that $L / R$ ratio, representing small intestinal permeability is increased, irrespective of possible confounding factors, in patients with diarrhea predominant IBS when compared to healthy controls. These findings point to a different pathophysiological mechanism in this IBS subtype. Furthermore, we have shown that adjustment for possible confounders is essential when studying intestinal permeability, especially in a heterogeneous disorder as IBS. 


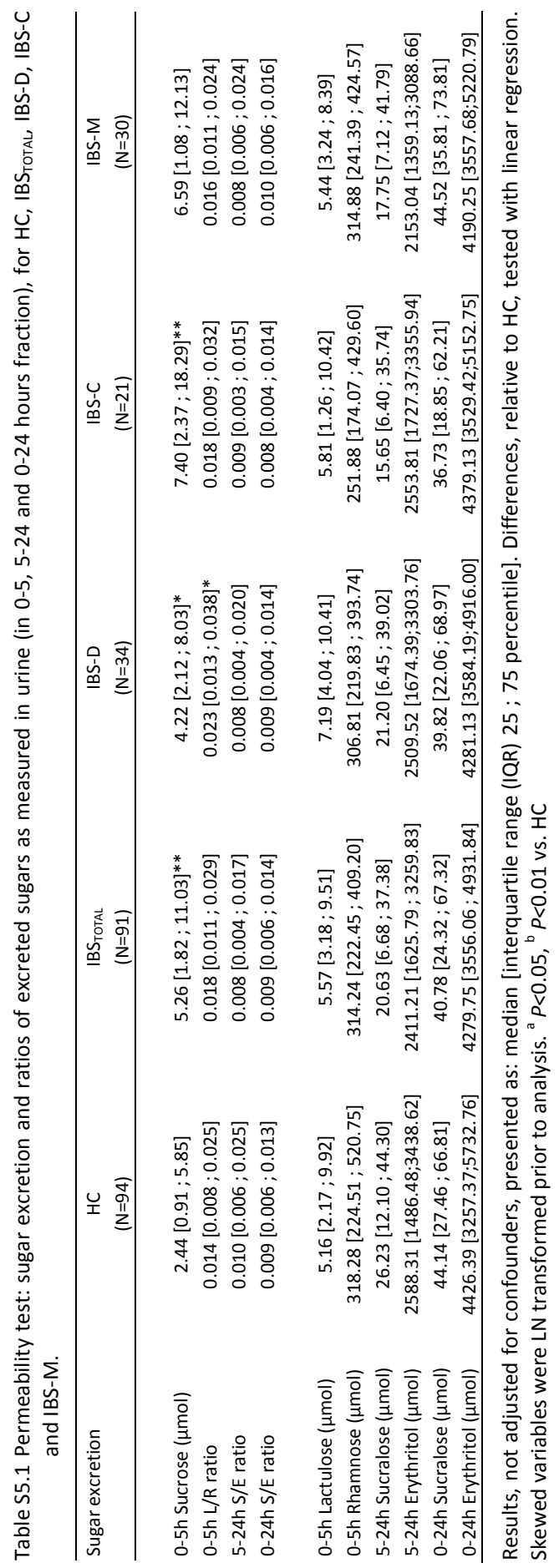




\section{References}

1. Simrén M, Brazier J, Coremans G, Dapoigny M, Müller-Lissner SA, Pace F, Smout AJ, Stockbrügger RW, Vatn $\mathrm{MH}$, Whorwell PJ. Quality of life and illness costs in irritable bowel syndrome. Digestion. 2004;69:254-61.

2. Spiegel BM. The burden of IBS: looking at metrics. Current gastroenterology reports. 2009;11:265-9.

3. Longstreth GF, Thompson WG, Chey WD, Houghton LA, Mearin F, Spiller RC. Functional bowel disorders. Gastroenterology. 2006;130:1480-91.

4. Camilleri M. Peripheral mechanisms in irritable bowel syndrome. N Engl J Med. 2012;367:1626-35.

5. Rao AS, Camilleri M, Eckert DJ, Busciglio I, Burton DD, Ryks M, Wong BS, Lamsam J, Singh R, Zinsmeister AR. Urine sugars for in vivo gut permeability: validation and comparisons in irritable bowel syndromediarrhea and controls. Am J Physiol Gastrointest Liver Physiol. 2011;301:G919-28.

6. Del Valle-Pinero AY, Van Deventer HE, Fourie NH, Martino AC, Patel NS, Remaley AT, Henderson WA.Gastrointestinal permeability in patients with irritable bowel syndrome assessed using a four probe permeability solution. Clin Chim Acta. 2013;418:97-101.

7. Dunlop SP, Hebden J, Campbell E, Naesdal J, Olbe L, Perkins AC, Spiller RC. Abnormal intestinal permeability in subgroups of diarrhea-predominant irritable bowel syndromes. Am J Gastroenterol. 2006;101:1288-94.

8. Marshall JK, Thabane M, Garg AX, Clark W, Meddings J, Collins SM; WEL Investigators. Intestinal permeability in patients with irritable bowel syndrome after a waterborne outbreak of acute gastroenteritis in Walkerton, Ontario. Aliment Pharmacol Ther. 2004;20:1317-22.

9. Spiller RC, Jenkins D, Thornley JP, Hebden JM, Wright T, Skinner M, Neal KR. Increased rectal mucosal enteroendocrine cells, $T$ lymphocytes, and increased gut permeability following acute Campylobacter enteritis and in post-dysenteric irritable bowel syndrome. Gut. 2000;47:804-11.

10. Zhou Q, Zhang B, Verne GN. Intestinal membrane permeability and hypersensitivity in the irritable bowel syndrome. Pain. 2009;146:41-6.

11. Zhou Q, Souba WW, Croce CM, Verne GN. MicroRNA-29a regulates intestinal membrane permeability in patients with irritable bowel syndrome. Gut. 2010;59:775-84.

12. Kerckhoffs AP, Akkermans LM, de Smet MB, Besselink MG, Hietbrink F, Bartelink IH, Busschers WB, Samsom M, Renooij W. Intestinal permeability in irritable bowel syndrome patients: effects of NSAIDs. Dig Dis Sci. 2010;55:716-23.

13. Gecse K, Róka R, Séra T, Rosztóczy A, Annaházi A, Izbéki F, Nagy F, Molnár T, Szepes Z, Pávics L, Bueno L, Wittmann T. Leaky gut in patients with diarrhea-predominant irritable bowel syndrome and inactive ulcerative colitis. Digestion. 2012;85:40-6.

14. Park JH, Park DI, Kim HJ, Cho YK, Sohn Cl, Jeon WK, Kim BI, Won KH, Park SM. The Relationship between Small-Intestinal Bacterial Overgrowth and Intestinal Permeability in Patients with Irritable Bowel Syndrome. Gut Liver. 2009;3:174-9.

15. Ersryd A, Posserud I, Abrahamsson H, Simren M. Subtyping the irritable bowel syndrome by predominant bowel habit: Rome II versus Rome III. Aliment Pharmacol Ther. 2007;26:953-61.

16. Meddings JB, Gibbons I. Discrimination of site-specific alterations in gastrointestinal permeability in the rat. Gastroenterology. 1998;114:83-92.

17. van Wijck K, van Eijk HM, Buurman WA, Dejong $\mathrm{CH}$, Lenaerts $\mathrm{K}$. Novel analytical approach to a multisugar whole gut permeability assay. Journal of chromatography B, Analytical technologies in the biomedical and life sciences. 2011;879:2794-801.

18. van Wijck K, Verlinden TJ, van Eijk HM, Dekker J, Buurman WA, Dejong $\mathrm{CH}$, et al. Novel multi-sugar assay for site-specific gastrointestinal permeability analysis: a randomized controlled crossover trial. Clinical nutrition. 2013;32:245-51.

19. Svedlund J, Sjodin I, Dotevall G. GSRS--a clinical rating scale for gastrointestinal symptoms in patients with irritable bowel syndrome and peptic ulcer disease. Dig Dis Sci. 1988;33: 129-34.

20. Thijssen AY, Jonkers DM, Leue C, van der Veek PP, Vidakovic-Vukic M, van Rood YR, Clemens $\mathrm{CH}$, Masclee AA. Dysfunctional cognitions, anxiety and depression in irritable bowel syndrome. J Clin Gastroenterol. 2010;44:e236-41. 
21. Zigmond AS, Snaith RP. The hospital anxiety and depression scale. Acta psychiatrica Scandinavica. 1983;67:361-70.

22. Bjelland I, Dahl AA, Haug TT, Neckelmann D. The validity of the Hospital Anxiety and Depression Scale. An updated literature review. J Psychosom Res. 2002;52:69-77.

23. Sutherland LR, Verhoef M, Wallace JL, Van Rosendaal G, Crutcher R, Meddings JB. A simple, noninvasive marker of gastric damage: sucrose permeability. Lancet. 1994;343:998-1000.

24. Pijls KE, Koek GH, Elamin EE, de Vries H, Masclee AA, Jonkers DM. Large intestine permeability is increased in patients with compensated liver cirrhosis. Am J Physiol Gastrointest Liver Physiol. 2014;306:G147-53.

25. Rutten EP, Lenaerts K, Buurman WA, Wouters EF. Disturbed intestinal integrity in patients with COPD; effects of activities of daily living. Chest. 2014;145:245-52.

26. Lambert GP, Schmidt A, Schwarzkopf K, Lanspa S. Effect of aspirin dose on gastrointestinal permeability. Int J Sports Med. 2012;33:421-5.

27. Troost FJ, Saris WH, Brummer RJ. Recombinant human lactoferrin ingestion attenuates indomethacininduced enteropathy in vivo in healthy volunteers. Eur J Clin Nutr. 2003;57: 1579-85.

28. Roberts A, Renwick AG, Sims J, Snodin DJ. Sucralose metabolism and pharmacokinetics in man. Food Chem Toxicol. 2000;38 Suppl 2:S31-41.

29. Arrigoni E, Brouns F, Amado R. Human gut microbiota does not ferment erythritol. Br J Nutr. 2005;94:643-6.

30. Suenaert P, Bulteel V, Den Hond E, Hiele M, Peeters M, Monsuur F, Ghoos Y, Rutgeerts P. The effects of smoking and indomethacin on small intestinal permeability. Aliment Pharmacol Ther. 2000;14:819-22.

31. Teixeira TF, Souza NC, Chiarello PG, Franceschini SC, Bressan J, Ferreira CL, Peluzio Mdo C. Intestinal permeability parameters in obese patients are correlated with metabolic syndrome risk factors. Clin Nutr. 2012;31:735-40.

32. Zhang $X$, Jiang $X$. Effects of enteral nutrition on the barrier function of the intestinal mucosa and Dopamine receptor expression in rats with traumatic brain injury. JPEN J Parenter Enteral Nutr. 2013 Sep 18. [Epub ahead of print]

33. Rummel W. [Biologic membrane functions in health and disease. Effects of bile acids and laxatives on the mucosal transfer]. Bull Schweiz Akad Med Wiss. 1976;32:233-50.

34. Mullin JM, Valenzano MC, Whitby M, Lurie D, Schmidt JD, Jain V, et al. Esomeprazole induces upper gastrointestinal tract transmucosal permeability increase. Aliment Pharmacol Ther. 2008;28:1317-25.

35. Gotteland M, Cruchet S, Frau V, Wegner ME, Lopez R, Herrera T, Sanchez A, Urrutia C, Brunser O. Effect of acute cigarette smoking, alone or with alcohol, on gastric barrier function in healthy volunteers. Dig Liver Dis. 2002;34:702-6.

36. Benoni $\mathrm{C}$, Prytz $\mathrm{H}$. Effects of smoking on the urine excretion of oral 51Cr EDTA in ulcerative colitis. Gut. 1998;42:656-8.

37. Martinez C, Gonzalez-Castro A, Vicario M, Santos J. Cellular and molecular basis of intestinal barrier dysfunction in the irritable bowel syndrome. Gut Liver. 2012;6:305-15.

38. Martínez C, Lobo B, Pigrau M, Ramos L, González-Castro AM, Alonso C, Guilarte M, Guilá M, de Torres I, Azpiroz F, Santos J, Vicario M. Diarrhoea-predominant irritable bowel syndrome: an organic disorder with structural abnormalities in the jejunal epithelial barrier. Gut. 2013;62:1160-8.

39. Barbara G, Stanghellini V, De Giorgio R, Cremon C, Cottrell GS, Santini D, Pasquinelli G, Morselli-Labate AM, Grady EF, Bunnett NW, Collins SM, Corinaldesi R. Activated mast cells in proximity to colonic nerves correlate with abdominal pain in irritable bowel syndrome. Gastroenterology. 2004;126: 693-702.

40. Turner JR. Intestinal mucosal barrier function in health and disease. Nat Rev Immunol. 2009;9:799-809.

41. Belmonte L, Beutheu Youmba S, Bertiaux-Vandaële N, Antonietti M, Lecleire S, Zalar A, Gourcerol G, Leroi AM, Déchelotte $P$, Coëffier M, Ducrotté $P$. Role of toll like receptors in irritable bowel syndrome: differential mucosal immune activation according to the disease subtype. PloS One. 2012;7:e42777.

42. Camilleri M, Madsen K, Spiller R, Greenwood-Van Meerveld B, Verne GN. Intestinal barrier function in health and gastrointestinal disease. Neurogastroenterol Motili. 2012;24:503-12. 
43. Gwee KA, Collins SM, Read NW, Rajnakova A, Deng Y, Graham JC, McKendrick MW, Moochhala SM. Increased rectal mucosal expression of interleukin 1beta in recently acquired post-infectious irritable bowel syndrome. Gut. 2003;52:523-6.

44. Chadwick VS, Chen W, Shu D, Paulus B, Bethwaite P, Tie A, Wilson I. Activation of the mucosal immune system in irritable bowel syndrome. Gastroenterology. 2002;122:1778-83.

45. Keszthelyi D. The serotonin precursor 5-hydroxytryptophan reinforces intestinal barrier function [abstract]. Gastroenterology. 2011 2011;140:S370.

46. Vanuytsel T, van Wanrooy S, Vanheel H, Vanormelingen C, Verschueren S, Houben E, Salim Rasoel S, Tóth J, Holvoet L, Farré R, Van Oudenhove L, Boeckxstaens G, Verbeke K, Tack J. Psychological stress and corticotropin-releasing hormone increase intestinal permeability in humans by a mast celldependent mechanism. Gut. 2013 Oct 23.

47. Barreau F, Cartier C, Leveque M, Ferrier L, Moriez R, Laroute V, Rosztoczy A, Fioramonti J, Bueno L. Pathways involved in gut mucosal barrier dysfunction induced in adult rats by maternal deprivation: corticotrophin-releasing factor and nerve growth factor interplay. J Physiol. 2007;580:347-56.

48. Demaude J, Salvador-Cartier C, Fioramonti J, Ferrier L, Bueno L. Phenotypic changes in colonocytes following acute stress or activation of mast cells in mice: implications for delayed epithelial barrier dysfunction. Gut. 2006;55:655-61.

49. Snoek SA, Verstege MI, Boeckxstaens GE, van den Wijngaard RM, de Jonge WJ. The enteric nervous system as a regulator of intestinal epithelial barrier function in health and disease. Expert Rev Gastroenterol Hepatol. 2010;4:637-51.

50. Bertiaux-Vandaele N, Youmba SB, Belmonte L, Lecleire S, Antonietti M, Gourcerol G, et al. The expression and the cellular distribution of the tight junction proteins are altered in irritable bowel syndrome patients with differences according to the disease subtype. Am J Gastroenterol. 2011;106: 2165-73. 


\section{Chapter}

The intestinal barrier in irritable bowel syndrome:

subtype-specific effects of the systemic compartment in an in vitro model

S Ludidi, DM Jonkers, EE Elamin, HJ Pieters, E Schaepkens, P Bours, J Kruimel, JM Conchillo, AA Masclee

PLoS One, provisionally accepted 


\section{Abstract}

\section{Background}

Irritable bowel syndrome (IBS) is a disorder with multifactorial pathophysiology. Intestinal barrier may be altered, especially in diarrhea-predominant IBS (IBS-D). Several mediators may contribute to increased intestinal permeability in IBS.

\section{Aim}

We aimed to assess effects of tryptase and LPS on in vitro permeability using a 3-dimensional cell model after basolateral cell exposure. Furthermore, we assessed the extent to which these mediators in IBS plasma play a role in intestinal barrier function.

\section{Materials and methods}

Caco-2 cells were grown in extracellular matrix to develop into polarized spheroids, and were exposed to tryptase $(10-50 \mathrm{mU})$, LPS $(1-50 \mathrm{ng} / \mathrm{ml})$ and two-fold diluted plasma samples of 7 patients with IBS-D, 7 with constipation-predominant IBS (IBS-C) and 7 healthy controls (HC). Barrier function was assessed by the flux of FITC-dextran (FD4) using live cell imaging. Furthermore, plasma tryptase and LPS were determined.

\section{Results}

Tryptase $(20$ and $50 \mathrm{mU})$ and LPS $(6.25-50 \mathrm{ng} / \mathrm{ml})$ significantly increased Caco-2 permeability versus control (all $P<0.05$ ). Plasma of IBS-D only showed significantly elevated median tryptase concentrations (7.1 [3.9-11.0] vs. $4.2[2.2-7.0]$ vs. $4.2[2.5-5.9] \mu \mathrm{g} / \mathrm{ml} ; P<0.05)$ and LPS concentrations (3.65 [3.00-6.10] vs. $3.10[2.60-3.80]$ vs. $2.65[2.40-3.40] \mathrm{EU} / \mathrm{ml} ; P<0.05)$ vs. IBS-C and HC. Also, plasma of IBS-D increased Caco- 2 permeability versus HC $(0.14450 \pm 0.00472$ vs. $0.00021 \pm 0.00003 ; P<0.001)$, which was attenuated by selective inhibiton of tryptase and LPS $(P<0.05)$.

\section{Conclusion}

Basolateral exposure of spheroids to plasma of IBS-D patients resulted in a significant increased FD4 permeation, which was partially abolished by selective inhibition of tryptase and LPS. These findings point to a role of systemic tryptase and LPS in the epithelial barrier alterations observed in patients with IBS-D. 


\section{Introduction}

Irritable bowel syndrome (IBS) is a functional gastrointestinal (GI) disorder, with an estimated prevalence of $10-15 \%$ in Western countries ${ }^{1}$. It is characterized by chronic abdominal pain or discomfort, and altered bowel habits in the absence of an organic cause $^{2}$.

IBS is a heterogeneous disorder of multifactorial origin with various disturbances along the gut-brain axis ${ }^{3}$. Recently, attention has been paid to low-grade chronic inflammation, changes in the composition of the intestinal microbiome ${ }^{4-7}$, motility and visceral perception ${ }^{3,8}$. Furthermore, alterations in the intestinal barrier have been reported in patients with $\mathrm{IBS}^{9,10}$ and were found to be associated with GI symptoms, such as diarrhea and abdominal pain ${ }^{5}$. It has been hypothesized that barrier dysfunction is an early event in IBS and may contribute to low-grade intestinal inflammation and increased visceral perception ${ }^{11}$. Studies that evaluated intestinal permeability in IBS subtypes reported that especially patients with diarrheapredominant IBS (IBS-D) $)^{12,13}$ and post-infectious (PI) IBS frequently show altered intestinal barrier function ${ }^{14}$.

Altered epithelial integrity may result from both intestinal and systemic factors. Inflammatory mediators, e.g. mast cell products, may contribute to impaired intestinal barrier function in IBS ${ }^{15}$. Tryptase (TRYP) is a serine protease, released by mast cells upon degranulation. An increased release of TRYP has been reported in patients with IBS and is more prominent in patients with IBS-D compared to patients with constipation predominant IBS (IBS-C) ${ }^{16-18}$. Luminal exposure to TRYP affects intestinal paracellular permeability both in vitro ${ }^{15}$ and ex vivo ${ }^{19}$. Recent data indicate that faecal supernatants and mucosal homogenates of IBS-D patients increase permeability in ex vivo models of intestinal tissue in Ussing chambers ${ }^{17,19}$.

Alterations in barrier function as observed in IBS, may give rise to permeation of bacteria and their products. Lipopolysaccharide (LPS), the major component of the cell wall of Gram-negative bacteria, is also able to affect barrier function. Under physiological conditions LPS will not pass the intact epithelium, but a reduced epithelial integrity may allow LPS to pass the GI barrier ${ }^{20,21}$.

Although both TRYP and LPS can be found in the systemic compartment, little is known about basolateral exposure relative to luminal exposure of these modulators in relation to barrier function. Furthermore, the question arises whether these systemic factors may be involved in the altered intestinal permeability in patients with IBS and secondly, whether differences exist between IBS subtypes. We hypothesize that TRYP and LPS in 
the systemic compartment contribute to the altered intestinal permeability as observed in IBS patients with diarrhea

Recently, we have shown that a three-dimensional (3D) cell culture model of Caco-2 spheroids represents a highly suitable physiological model to investigate basolateral exposure of substances on paracellular permeability ${ }^{22,23}$. Aim of the present explorative study was 1) to evaluate the effect of basolateral exposure of TRYP and LPS on intestinal permeability in the 3D intestinal cell culture model 2) to assess TRYP and LPS plasma levels in patients with IBS-D and IBS-C versus healthy controls (HC) 3) to investigate the effect of plasma of IBS-D and IBS-C patients on intestinal permeability in vitro and 4) whether such an effect is TRYP and/or LPS related.

\section{Materials and methods}

\section{Cell line, culture conditions and three-dimensional (3D) cell culture}

Colonic adenocarcinoma cell line (Caco-2) from the American Type Culture Collection (ATCC, Rockville, USA) were maintained in Dulbecco's Modified Eagle Medium (DMEM; Lonza Benelux BV, Breda, the Netherlands) and cultured in 3D as described previously ${ }^{22}$. Briefly, Caco- 2 cells ( $4 \times 10^{4}$ cells/dish; passage 26-36) were embedded in $40 \%$ growth factor-reduced Matrigel ${ }^{\circledR}(8 \mathrm{mg} / \mathrm{ml}$; BD Biosciences, San José, CA-USA) and solidified at $37^{\circ} \mathrm{C}$ for $30 \mathrm{~min}$ in glass bottom culture dishes. Thereafter, medium was added and spheroids were allowed to form over $5-7$ days at $37^{\circ} \mathrm{C}^{22}$.

\section{Exposure to TRYP and LPS and determination of intestinal epithelial barrier function}

3D spheroids were exposed basolaterally to TRYP concentrations of 10, 20 and $50 \mathrm{mU}$ (Sigma-Aldrich, Chemie B.V., Zwijndrecht, The Netherlands), LPS concentrations of 1, 6,25, 12.5, 25 and $50 \mathrm{ng} / \mathrm{ml}$ (Sigma-Aldrich) and combinations of TRYP and LPS (10 mU TRYP $+6.25 \mathrm{ng} / \mathrm{ml}$ LPS and $20 \mathrm{mU}$ TRYP $+12.5 \mathrm{ng}$ LPS) in the presence of $10 \%(\mathrm{v} / \mathrm{v})$ fluorescein isothiocyanate-labelled dextran of $4 \mathrm{kDa}$ (FD4; Sigma-Aldrich) at $37^{\circ} \mathrm{C}$ for 24 hours. Two mM ethylene glycol tetra acetic acid (EGTA) to induce maximum TJs disruption and growth medium only were used as positive and negative control, respectively. Permeability of the spheroids was determined by the flux of FD4 from the basal to the luminal compartment (i.e., L/BL ratio) using confocal microscopy as described previously ${ }^{23}$. Confocal images were taken with Leica TCS SPE confocal laser scanning microscope (Leica Microsystems $\mathrm{GmbH}$, Mannheim, Germany) and processed using TCS SPE browser and Image J software ${ }^{24}$. 
Thereafter, spheroids were exposed to $\operatorname{TRYP}(10,20$ and $50 \mathrm{mU})$ or LPS $(1,6.25$ and $12.5 \mathrm{ng} / \mathrm{ml}$ ) in the presence of either $100 \mu \mathrm{M}$ TRYP inhibitor nafamostat mesylate $(\mathrm{NM})^{19}$ or LPS inhibitor polymyxin B sulphate salt (PMB) at $10,62.5$ or $125 \mathrm{ng} / \mathrm{ml}^{25}$, respectively. Spheroids were also exposed to TRYP and LPS in combination with NM and $\mathrm{PMB}$, followed by assessment of basal to luminal permeation of FD4.

\section{Assessment of cell viability}

Cell viability was determined by measuring release of lactate dehydrogenase (LDH), an indicator of plasma membrane integrity, using the LDH assay (CytoTox ONE ${ }^{\text {tm }}$ Homogeneous Membrane Integrity Assay; Promega, the Netherlands). Briefly, Caco-2 cells were cultured in 3D in 96-well plates and incubated with $200 \mu \mathrm{L}$ of solutions containing LPS (all concentrations), TRYP (all concentrations) and the combination of TRYP and LPS (i.e.10 mU TRYP in combination with $1 \mathrm{ng} / \mathrm{ml}$ LPS and $20 \mathrm{mU}$ TRYP in combination with $6.25 \mathrm{ng} / \mathrm{ml}$ LPS) with and without their respective inhibitors. Plates were incubated at $37^{\circ} \mathrm{C}$ for 24 hours and equilibrated at room temperature (RT) for $20 \mathrm{~min}$. Then of the substrate mix was added and incubated at RT, protected from light for another 30 minutes, followed by addition of $20 \mu \mathrm{l}$ stop solution. Maximum LDH release was induced by using $200 \mu \mathrm{l}$ of $1 \%$ (v/v) Triton with $99 \%$ (v/v) PBS (SigmaAldrich, Chemie B.V., Zwijndrecht-The Netherlands). The fluorescence was measured at an excitation and an emission wavelength of $560 \mathrm{~nm}$ and $590 \mathrm{~nm}$, respectively. The percentage of LDH activity was calculated as percentage of maximum LDH release and compared with negative control.

\section{Study population and sample collection}

Patients with clearly defined IBS-D ( $N=7)$ and IBS-C $(N=7)$ subtypes, according to the Rome III criteria, and $7 \mathrm{HC}$ were included in the present study. From each subject, blood samples were collected in pre-cooled Sodium-Heparin tubes and immediately placed on ice. Within 2 hours, plasma samples were centrifuged (4000 rpm; $4^{\circ} \mathrm{C}$ ) after which the supernatants were collected and stored at $-80^{\circ} \mathrm{C}$ until further experiments.

The study was part of a large prospective cohort study in IBS patients. All subjects had signed an informed consent prior to participation. The study protocol has been approved by the Maastricht University Medical Centre+ Committee of Ethics and was executed according to the Declaration of Helsinki $\left(59^{\text {th }}\right.$ general assembly of the WMA, Seoul, South Korea, Oct. 2008). The study has been registered in the US National Library of Medicine (http://www.clinicaltrials.gov, NCT00775060). 


\section{Quantification of TRYP and LPS concentrations in plasma}

Total TRYP levels in plasma were measured using the ImmunoCAP ${ }^{\circledR}$ (Phadia AD, Upsala, Sweden). The enzyme-based Limulus amoebocyte lysate chromomeric endpoint assay (Hycult $^{\circledR}$ Biotech, Uden, The Netherlands) was used to measure plasma LPS concentrations. Both were performed according to manufacturer's instructions.

\section{Exposure of human plasma on intestinal epithelial barrier function in vitro}

To assess barrier function, 3D spheroids were incubated basolaterally with the plasma samples of the IBS-D, IBS-C and HC. Medium only and $2 \mathrm{mM}$ ethylene glycol tetra-acetic acid (EGTA) were used as negative and positive controls, respectively. Spheroids were incubated with $2 \mathrm{ml}$ containing $37.5 \%(\mathrm{v} / \mathrm{v})$ plasma, 52.5\% (v/v) medium and $10 \%(\mathrm{v} / \mathrm{v})$ FD4. The basal to luminal FD4 permeation was assessed by confocal microscopy as described above.

Subsequently, effects of NM $(100 \mu \mathrm{M})$ and PMB $(62.5 \mathrm{ng} / \mathrm{ml})$ were assessed on in vitro permeability after co-incubation with plasma of the IBS-D patients.

\section{Data analyses}

All experiments were performed in triplicate and results were reported as mean \pm SEM of at least 5 spheroids per experiment and $7 \mathrm{z}$ stack measurements per spheroid. In vitro experiments were performed with plasma samples of 7 subjects in each subgroup (i.e. HC vs. IBS-D vs. IBS-C). Permeability data were analysed parametrically using a oneway analysis of variance (ANOVA) and Tukey's post-hoc test was performed to determine significant differences between different treatment conditions (GraphPad Software Incorporated, CA, USA - version 6.0; Macintosh).

Serine protease activity and LPS concentrations in plasma were compared between groups using a Kruskal-Wallis test. A $P$-value under 0.05 was considered statistically significant. A Bonferroni correction for multiple testing was applied to all data.

\section{Results}

\section{Subjects}

IBS patients were selected based on IBS-subtype (IBS-D ( $N=7)$ or IBS-C $(\mathrm{N}=7)$ ) and compared with $\mathrm{HC}(\mathrm{N}=7)$. The IBS groups did not differ significantly with regards to disease duration and age, but female sex was significantly more prevalent in IBS-C versus IBS-D ( $85.7 \%$ vs. $28.6 \%$, respectively; $P<0.05)$. No differences were found in age and gender comparing $\mathrm{HC}$ with the IBS subgroups. 


\section{FD4 permeation after basolateral exposure to TRYP and LPS}

TRYP at $20 \mathrm{mU}$ and $50 \mathrm{mU}$ induced a significant increase of the basal to luminal FD4 flux (Figure 6.1a, $P<0.01$ and $P<0.001$, respectively). A non-significant increase was found for $10 \mathrm{mU}$ TRYP $(P=0.076)$.

Exposure to LPS also increased FD4 permeation at concentrations of $6.25 \mathrm{ng} / \mathrm{ml}$ $(P<0.05), 12.5 \mathrm{ng} / \mathrm{ml}, 25 \mathrm{ng} / \mathrm{ml}$ and $50 \mathrm{ng} / \mathrm{ml}$ (all $P<0.01$ ), but no effect was found for $1 \mathrm{ng} / \mathrm{ml}$ (Figure 6.1b). Furthermore, when compared to negative control, the combination of $10 \mathrm{mU}$ TRYP with $6.25 \mathrm{ng} / \mathrm{ml}$ LPS as well as $20 \mathrm{mU}$ TRYP with $12.5 \mathrm{ng} / \mathrm{ml}$ LPS significantly increased the L/BL FD4 ratio versus negative control (Figure 6.1c; both $P<0.001)$, which was also significantly higher compared to each of the stressors separately $(P<0.01)$, pointing to an additive effect of TRYP and LPS combined.
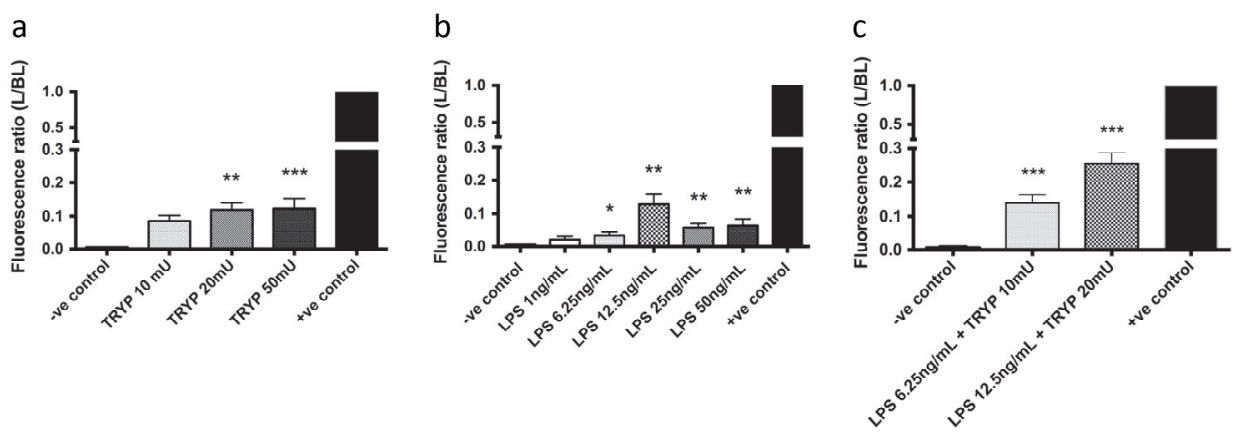

Figure 6.1 Permeation of FD4 (mean \pm SEM) after exposure to different concentrations of TRYP (a); LPS (b) and combination of TRYP and LPS (c). ${ }^{*} P<0.05$; ${ }^{* *} P<0.01 ; * * * P<0.001$ compared to negative control (medium only). Analyses were based on at least 5 spheroids per experiment. Experiments were carried out in 3-fold.

\section{TRYP and LPS levels in plasma}

Median TRYP levels were significantly higher in plasma of IBS-D versus $\mathrm{HC}$ and IBS-C (Figure 6.2b; 7.1 [3.9-11.0] vs. 4.2 [2.5-5.9] vs. 4.2 [2.2-7.0] ng/ml, respectively; $P<0.05)$. Furthermore, LPS levels were significantly increased in plasma of IBS-D patients compared to $\mathrm{HC}$ (3.65 [3.00-6.10] vs. 2.65 [2.40-3.40] EU/ml, respectively; $P<0.05)$, but not compared to IBS-C (3.10 [2.60-3.80] EU/ml; N.S.). No significant differences were found between IBS-C and HC (Figure 6.2b).

\section{Inhibition of TRYP and LPS on FD4 permeation}

Compared to $20 \mathrm{mU}$ TRYP alone, adding the TRYP inhibitor NM (100 $\mu \mathrm{M})$ significantly attenuated the TRYP-induced increase of the L/BL FD4 ratio $(0.0541 \pm 0.013$ vs. $0.1139 \pm 0.021 ; P<0.05)$. A similar effect was observed after exposure to $50 \mathrm{mU}$ TRYP 
with $100 \mu \mathrm{M}$ NM versus $50 \mathrm{mU}$ TRYP only $(0.0513 \pm 0.012$ vs. $0.1231 \pm 0.029 ; P<0.01)$. No significant effect was observed when adding $100 \mu \mathrm{M}$ NM to $10 \mathrm{mU}$ TRYP.

Concentrations up to $25 \mathrm{ng} / \mathrm{ml}$ LPS were tested for co-incubation with PMB. Only simultaneous exposure to $12.5 \mathrm{ng} / \mathrm{ml}$ LPS with $125 \mathrm{ng} / \mathrm{ml}$ LPS inhibitor PMB, resulted in a significant decrease versus $12.5 \mathrm{ng} / \mathrm{ml}$ LPS alone $(0.0534 \pm 0.010$ vs. $0.128 \pm 0.030$; $P<0.05)$. No significant effects were observed for the other concentrations of LPS after co-incubation with their respective concentrations of $\mathrm{PMB}$.

A slight but significant increase in FD4 permeation was also found by NM and by PMB alone compared to the negative control $(0.0737 \pm 0.025$ and $0.0358 \pm 0.023$, respectively, versus $0.00424 \pm 0.0011$; both $P<0.0001$ ).

a

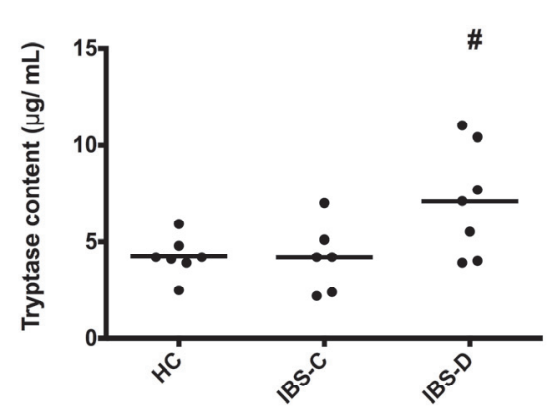

b

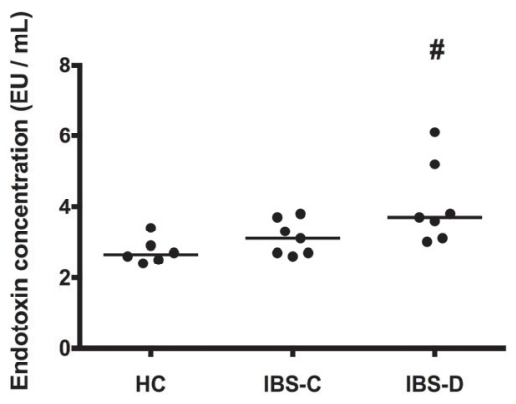

Figure 6.2 Median plasma TRYP (a) and LPS levels (b) in healthy controls ( $\mathrm{HC}, \mathrm{N}=7$ and $\mathrm{N}=6$, respectively), constipation-predominant (IBS-C, N=7) and diarrhea-predominant IBS patients (IBS-D, N=7); a. $\# P<0.05$ compared to $\mathrm{HC}$ and IBS-C; b. \#P<0.05 compared to $\mathrm{HC}$ only. Experiments were carried out in 3-fold.

\section{TRYP, LPS and cell viability}

No significant increase in LDH release as indicator of plasma membrane integrity, hence cell viability, was detected after incubation with TRYP (10 and $20 \mathrm{mU}$ ), LPS (1 to $25 \mathrm{ng} / \mathrm{ml}$, nor for, the combination of TRYP and LPS with or without their respective inhibitors NM and PMB (all $P>0.05$ ).

\section{FD4 permeation in 3D spheroids after exposure to plasma}

In Figure 6.3, the FD4 flux after basolateral exposure to plasma has been shown. Spheroids exposed to plasmas of IBS-D as well as IBS-C showed significant increased L/BL FD4 ratios in comparison to HC. The magnitude of the effect was significantly 
larger in IBS-D, showing increased L/BL FD4 ratios in comparison with plasma of IBS-C $(P<0.0001)$.

Furthermore, the increased FD4 permeation found after exposure to plasma of IBS-D patients, was significantly reduced after co-incubation with NM (100 $\mu \mathrm{M})$, PMB $(62.5 \mathrm{ng} / \mathrm{ml}$ ) or the combination of both (Figure 6.4: all $P<0.0001)$.

a

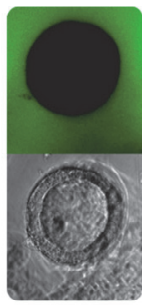

$\mathrm{HC}$

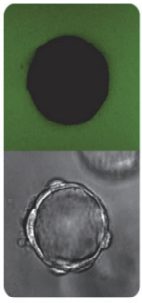

IBS-C

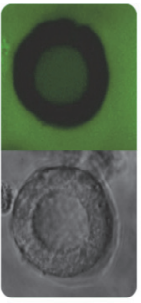

IBS-D

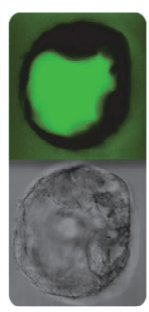

+ve control b

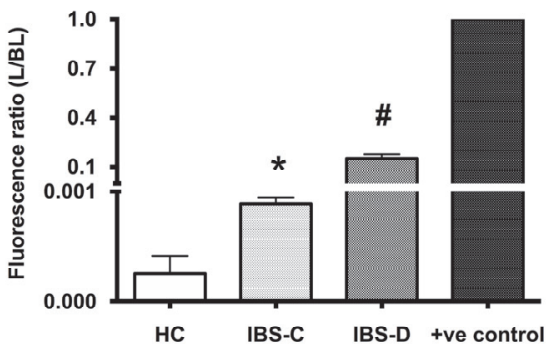

Figure 6.3 Permeation of FD4 after exposure to plasma of healthy controls ( $\mathrm{HC}, \mathrm{N}=7)$, constipationpredominant IBS (IBS-C, N=7), diarrhea-predominant IBS (IBS-D, N=7) and positive control, (+ve control i.e. EGTA) using confocal microscopy (a) and expressed as mean $\pm \mathrm{SEM} \mathrm{L} / \mathrm{BL}$ fluorescence ratio of triplicate experiments (b) $* P<0.0001$ vs. $\mathrm{HC} \# P<0.0001$ vs. HC and IBS-C. Experiments were carried out in 3-fold.

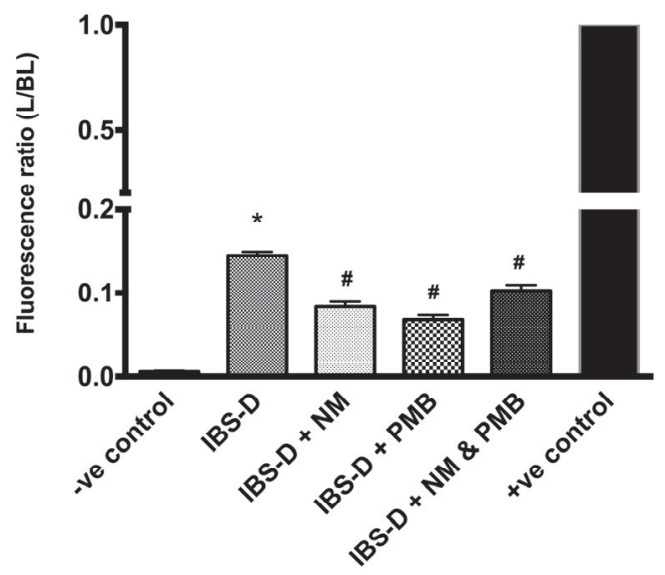

Figure 6.4 Permeation of FD4 (mean \pm SEM), expressed as L/BL fluorescence ratio after exposure to plasma of patients with diarrhea-predominant IBS (IBS-D); plasma of IBS-D with 100uM nafamostat mesylate (NM); plasma of IBS-D with $62.25 \mathrm{ng} / \mathrm{ml}$ polymyxin $B(P M B)$ and a combination of plasma, $\mathrm{NM}$ and $\mathrm{PMB}\left({ }^{*} P<0.0001\right.$ vs. $\mathrm{HC} ; \# P<0.0001$ vs. IBS-D). Experiments were carried out in 3-fold. 


\section{Discussion}

Using the 3D intestinal cell culture model, we found that basolateral exposure to both tryptase and LPS induced a significant increase in FD4 permeation. Plasma LPS and tryptase levels were increased IBS-D, but not in the IBS-C patients. A significant increase in FD4 permeation was observed when 3D spheroids were exposed to plasma of IBS-D compared to IBS-C patients and controls. These effects were partially abolished by adding tryptase- and LPS-inhibitors.

Recent data suggest that local inflammation is associated with alterations in intestinal barrier function ${ }^{13,26,27}$. Low-grade intestinal inflammation has repeatedly been demonstrated in IBS $^{28}$ and findings point to a subtype- specific increase of tryptase in mucosal and faecal samples ${ }^{16,18,29}$ and to a key role for mast cells and tryptase in the altered barrier function of IBS-D patients ${ }^{17,19}$. Tryptase can induce signal transduction via protein activated receptors (PAR), which are abundantly expressed in intestinal epithelium ${ }^{30,31}$. Their activation may result in increased paracellular permeability and visceral perception ${ }^{17}$.

Whereas most studies focused on apical or luminal intestinal cell surface exposure, little is known on basolateral exposure. Up to now Lee et al. were the only group to report that tryptase increased permeability when added to the basolateral surface of intestinal biopsies of healthy subjects ${ }^{19}$.

We employed a three-dimensional Caco-2 cell culture model to promote a physiologically relevant microenvironment mimicking the specificity of tissues in vivo, with fully polarized cells including formation of tight and adherens junctions and a central lumen. Unlike the conventional two-dimensional, cultures in extracellular matrix (ECM) proteins form 3D spheroids and re-establish cell-ECM, cell-cell interactions and signaling pathways involved in cell differentiation, proliferation and barrier function ${ }^{32}$. Currently 3D cultures are widely used in cell biology studies, including cell adhesion, cell migration, tumour biology, and epithelial morphogenesis ${ }^{33}$. The model is found to be highly suitable to study epithelial barrier function after basolateral exposure ${ }^{22,23}$. The overall results were in line with those obtained by the $2 \mathrm{D}$ model $^{34,35}$.

Using the 3D sphehoids, we were able to show that tryptase and LPS, even at low concentrations (i.e $20 \mathrm{mU}$ tryptase and $6.25-12.5 \mathrm{ng} / \mathrm{ml}$ LPS) can result in increased FD4 permeation, indicating enhanced paracellular permeability. In addition, we found a significant increase in FD4 permeation after exposure to plasma of both IBD-D and IBS-C patients compared to HC, with a significantly higher effect for IBS-D versus IBS-C patients. Using this approach, we could confirm the in vitro findings by Wilcz-Vellega et 
$a l^{36}$ and by Lee et al. using biopsies of healthy subjects mounted in Ussing chambers ${ }^{19}$. Moreover, we expanded on their findings by demonstrating for the first time significantly elevated tryptase and LPS concentrations in plasma of IBS-D patients comparison to HC. Furthermore, plasma tryptase was also significantly increased in IBSD compared to IBS-C patients. Our data indicate that plasma tryptase may contribute to the changes in barrier function observed after exposure to plasma of IBS-D patients, since selective inhibition of plasma tryptase significantly reduced intensity of FD4 permeation. Increased numbers and/or activity of mast cells as observed in patients with IBS-D, may reflect tryptase release and spill-over from the intestinal compartment into the systemic circulation, contributing to the observed differences between IBS-D, $\mathrm{HC}$ and IBS- $\mathrm{C}^{37}$.

The second mediator we investigated was basolateral exposure to LPS, which significantly and dose-dependently increased FD4 permeation (from the basolateral side into the lumen). This observation is in line with in vitro studies showing increased permeability of Caco-2 monolayers after basolateral exposure to high $(100-500 \mu \mathrm{g} / \mathrm{ml})^{38}$ but also low $(0.3-20 \mathrm{ng} / \mathrm{ml})^{39}$ concentrations of LPS. Remarkably, $12.5 \mathrm{ng} / \mathrm{ml}$ LPS caused a significant peak in FD4 permeation in all experiments performed in triplicate. It is not known what causes this dose-specific difference. An effect on cell viability was unlikely since LDH leakage was not significantly affected at any of the LPS concentrations used.

Elevated LPS concentrations (i.e. about 1.5-fold increase) have previously been found in patients with inflammatory bowel disease when compared to healthy subjects, and were also found to be related to disease activity ${ }^{40}$. In the present study, circulating LPS concentrations were also found to be significantly increased 1.5 fold in IBS-D patients compared to HC. Although LPS can permeate to the systemic compartment as a consequence of a disturbed barrier function, it is important to note that LPS present in the circulation can also affect barrier function and contribute to sustain an increased permeability from the basolateral side. In line with our observations and hypothesis, O'Dwyer et al. demonstrated that a single dose of $4 \mathrm{ng} / \mathrm{ml}$ LPS infused intravenously was able to increase intestinal permeability in healthy subjects ${ }^{41}$.

Although concentrations for tryptase and LPS were increased in IBS-D, they were still within the physiological range. However, the combination of tryptase and LPS was found to induce a more pronounced effect on in vitro permeability than each mediator separately, pointing to an additive effect. This may (in part) explain the significant increase in BL/L FD4 flux after adding plasma of IBS-D, despite the relative low concentrations of tryptase and LPS. When selectively inhibiting tryptase and LPS after plasma exposure, permeability showed a significant, but incomplete decrease. This might be the result of a relative overdose of nafamostat mesylate, as that the tryptase 
inhibitor alone showed an effect on the BL/L FD4 flux. Furthermore, we should acknowledge the influence of other yet not identified mediators.

Plasma of IBS-D patients resulted in markedly and significantly increased FD4 permeation compared to HCS and IBS-C patients, which, at least partially, was related to elevated tryptase and LPS levels.. However, the plasma of IBS-C patients also showed a significant but much smaller increase in FD4 permeation when added to the 3D spheroids. LPS and tryptase levels were not found to be increased in these patients, suggesting that other factors, such as cysteine protease activity ${ }^{16}$ may have induced the observed effect. Considering the rather small increment in permeability caused by plasma of IBS-C patients in our study, we think it is unlikely that substantial cysteine protease activity could have contributed to the permeability alterations observed in neither IBS-C nor IBS-D patients. These data encourages the hypothesis that the IBS subtypes with predominant diarrhea and constipation are different entities, not only in clinical presentation, but also regarding the underlying pathophysiological mechanisms.

One of the major weaknesses of our study is that we did not take into account the potential effect of medication that may affect permeability ${ }^{42}$, such as proton pump inhibitors (PPI) and non-steroidal anti-inflammatory drugs (NSAIDs) medications ${ }^{43}$. Although all subjects participating in our study were asked to refrain from any medication 24 hours before blood collection, chronic or long-term medication intake may have had an effect on intestinal permeability in our patients as well.

The strength of the present study is that we aimed to translate the in vitro permeability findings after basolateral exposure of tryptase and LPS, to the in vivo situation by incubating the 3D spheroids with plasma samples of patients and controls and selectively blocking potential mediators to confirm their contributing role. Although it has to be acknowledged than only a limited number of subjects were included per group, the experiments were performed in triplicate and all individual plasma samples of IBS-D patients induced an increased intestinal permeability. However, one must realize that effects observed in Caco- 2 cells cannot be one to one extrapolated to the in vivo situation. Nonetheless, since cell-to-cell interaction is crucial in epithelial cell biology ${ }^{44}$, the 3D model provides a more realistic microenvironment to simulate in vivo responses to stressors compared to 2-dimensional monolayers ${ }^{22,45}$. Furthermore, in the present study, all analyses were done at a functional level by measuring FD4 permeation as indicator of paracellular permeability. No structural analyses have been performed investigating for example the localization and expression of tight junction (TJ) proteins. As tryptase and LPS can induce TJ re-localization ${ }^{17,39}$, analyses of TJs and signaling cascades may provide additional insight in the mechanisms involved. 
In summary, we demonstrated that basolateral exposure of a 3D cell model to tryptase and LPS significantly altered FD4 permeation. In plasma of patients with IBS-D, circulating tryptase and LPS were significantly increased andexposure of the spheroids to plasma of these patients resulted in increased FD4 permeation. The magnitude of the increased permeability was significantly more pronounced in IBS-D than IBS-C patients and selective inhibition of tryptase and LPS partially abolished the effect. These data point to a role of systemic tryptase and LPS in epithelial barrier dysfunction in patients with IBS-D. 


\section{References}

1. Longstreth GF, Thompson WG, Chey WD, Houghton LA, Mearin F, Spiller RC. Functional bowel disorders. Gastroenterology. 2006;130:1480-91.

2. Drossman DA, Camilleri M, Mayer EA, Whitehead WE. AGA technical review on irritable bowel syndrome. Gastroenterology. 2002;123:2108-31.

3. Azpiroz F, Bouin M, Camilleri M, Mayer EA, Poitras P, Serra J, Spiller RC. Mechanisms of hypersensitivity in IBS and functional disorders. Neurogastroenterol Motil. 2007;19(1 Suppl):62-88.

4. Cremon C, Gargano L, Morselli-Labate AM, Santini D, Cogliandro RF, De Giorgio R, Stanghellini V, Corinaldesi R, Barbara G. Mucosal immune activation in irritable bowel syndrome: gender-dependence and association with digestive symptoms. Am J Gastroenterol. 2009;104:392-400.

5. Barbara G, Stanghellini V, De Giorgio R, Cremon C, Cottrell GS, Santini D, Pasquinelli G, Morselli-Labate AM, Grady EF, Bunnett NW, Collins SM, Corinaldesi R. Activated mast cells in proximity to colonic nerves correlate with abdominal pain in irritable bowel syndrome. Gastroenterology. 2004;126: 693-702.

6. Ringel $\mathrm{Y}$, Maharshak $\mathrm{N}$. The intestinal microbiota and immune function in the pathogenesis of irritable bowel syndrome. Am J Physiol Gastrointestinal Liver Physiol. 2013;305:G529-41.

7. Chadwick VS, Chen W, Shu D, Paulus B, Bethwaite P, Tie A, Wilson I. Activation of the mucosal immune system in irritable bowel syndrome. Gastroenterology. 2002;122:1778-83.

8. Mertz H, Naliboff B, Munakata J, Niazi N, Mayer EA. Altered rectal perception is a biological marker of patients with irritable bowel syndrome. Gastroenterology. 1995;109:40-52.

9. Camilleri M, Lasch K, Zhou W. Irritable bowel syndrome: methods, mechanisms, and pathophysiology. The confluence of increased permeability, inflammation, and pain in irritable bowel syndrome Am J Physiol Gastrointest liver Physiol. 2012;303:G775-85.

10. Barbara G. Mucosal barrier defects in irritable bowel syndrome. Who left the door open? Am J Gastroenterol. 2006;101:1295-8.

11. Bertiaux-Vandaele N, Youmba SB, Belmonte L, Lecleire S, Antonietti M, Gourcerol G, Leroi AM, Dechelotte P, Menard JF, Ducrotte P, Coeffier M. The expression and the cellular distribution of the tight junction proteins are altered in irritable bowel syndrome patients with differences according to the disease subtype. Am J Gastroenterol. 2011;106:2165-73.

12. Dunlop SP, Hebden J, Campbell E, Naesdal J, Olbe L, Perkins AC, Spiller RC. Abnormal intestinal permeability in subgroups of diarrhea-predominant irritable bowel syndromes. Am J Gastroenterol. 2006;101:1288-94.

13. Zhou $\mathrm{Q}$, Zhang $\mathrm{B}$, Verne GN. Intestinal membrane permeability and hypersensitivity in the irritable bowel syndrome. Pain. 2009;146:41-6.

14. Marshall JK, Thabane M, Garg AX, Clark W, Meddings J, Collins SM, Investigators WEL. Intestinal permeability in patients with irritable bowel syndrome after a waterborne outbreak of acute gastroenteritis in Walkerton, Ontario. Aliment Pharmacol Ther. 2004;20:1317-22.

15. Ossovskaya VS, Bunnett NW. Protease-activated receptors: contribution to physiology and disease. Physiol Rev. 2004 Apr;84(2):579-621.

16. Annahazi A, Ferrier L, Bezirard V, Leveque $M$, Eutamene $H$, Ait-Belgnaoui $A$, Coeffier $M$, Ducrotte $P$, Roka R, Inczefi O, Gecse K, Rosztoczy A, Molnar T, Ringel-Kulka T, Ringel Y, Piche T, Theodorou V, Wittmann T, Bueno L. Luminal Cysteine-Proteases Degrade Colonic Tight Junction Structure and Are Responsible for Abdominal Pain in Constipation-Predominant IBS. Am J Gastroenterol. 2013;108: 1322-31.

17. Gecse K, Roka R, Ferrier L, Leveque M, Eutamene H, Cartier C, Ait-Belgnaoui A, Rosztoczy A, Izbeki F, Fioramonti J, Wittmann T, Bueno L. Increased faecal serine protease activity in diarrhoeic IBS patients: a colonic lumenal factor impairing colonic permeability and sensitivity. Gut. 2008;57:591-9.

18. Roka R, Rosztoczy A, Leveque M, Izbeki F, Nagy F, Molnar T, Lonovics J, Garcia-Villar R, Fioramonti J, Wittmann T, Bueno L. A pilot study of fecal serine-protease activity: a pathophysiologic factor in diarrhea-predominant irritable bowel syndrome. Clin Gastroenterol Hepatol. 2007;5:550-5. 
19. Lee JW, Park JH, Park DI, Park JH, Kim HJ, Cho YK, Sohn Cl, Jeon WK, Kim BI. Subjects with diarrheapredominant IBS have increased rectal permeability responsive to tryptase. Dig Dis Sci. 2010;55:2922-8.

20. Benoit R, Rowe S, Watkins SC, Boyle P, Garrett M, Alber S, Wiener J, Rowe MI, Ford HR. Pure endotoxin does not pass across the intestinal epithelium in vitro. Shock. 1998;10:43-8.

21. Ge Y, Ezzell RM, Warren HS. Localization of endotoxin in the rat intestinal epithelium. J Infect Dis. 2000; 182:873-81.

22. Elamin E, Jonkers D, Juuti-Uusitalo K, van ljzendoorn S, Troost F, Duimel H, Broers J, Verheyen F, Dekker J, Masclee A. Effects of ethanol and acetaldehyde on tight junction integrity: in vitro study in a three dimensional intestinal epithelial cell culture model. PloS One. 2012;7:e35008.

23. Elamin E, Masclee A, Juuti-Uusitalo K, van Ijzendoorn S, Troost F, Pieters HJ, Dekker J, Jonkers D. Fatty acid ethyl esters induce intestinal epithelial barrier dysfunction via a reactive oxygen speciesdependent mechanism in a three-dimensional cell culture model. PloS One. 2013;8:e58561.

24. Collins TJ. Image for microscopy. Biotechniques. 2007;43(1 Suppl):25-30.

25. Cuellar A, Fonseca A, Gomez A. [Effect of lipopolysaccharides on human dendritic cell cultures and its inhibition by polymyxin B]. Biomedica. 2004;24:413-22.

26. Zhou Q, Souba WW, Croce CM, Verne GN. MicroRNA-29a regulates intestinal membrane permeability in patients with irritable bowel syndrome. Gut. 2010;59:775-84.

27. Spiller RC, Jenkins D, Thornley JP, Hebden JM, Wright T, Skinner M, Neal KR. Increased rectal mucosal enteroendocrine cells, T lymphocytes, and increased gut permeability following acute Campylobacter enteritis and in post-dysenteric irritable bowel syndrome. Gut. 2000;47:804-11.

28. Matricon J, Meleine M, Gelot A, Piche T, Dapoigny M, Muller E, Ardid D. Review article: Associations between immune activation, intestinal permeability and the irritable bowel syndrome. Aliment Pharmacol Ther. 2012;36:1009-31.

29. Lee H, Park JH, Park DI, Kim HJ, Cho YK, Sohn Cl, Jeon WK, Kim BI, Chae SW. Mucosal mast cell count is associated with intestinal permeability in patients with diarrhea predominant irritable bowel syndrome. J Neurogastroenterol Motil. 2013;19:244-50.

30. Amadesi S, Nie J, Vergnolle N, Cottrell GS, Grady EF, Trevisani M, Manni C, Geppetti P, McRoberts JA, Ennes H, Davis JB, Mayer EA, Bunnett NW. Protease-activated receptor 2 sensitizes the capsaicin receptor transient receptor potential vanilloid receptor 1 to induce hyperalgesia. J Neurosci. 2004;24: 4300-12.

31. Bohm SK, Kong W, Bromme D, Smeekens SP, Anderson DC, Connolly A, Kahn M, Nelken NA, Coughlin SR, Payan DG, Bunnett NW. Molecular cloning, expression and potential functions of the human proteinase-activated receptor-2. Biochem J. 1996;314:1009-16.

32. Maltman DJ, Przyborski SA. Developments in three-dimensional cell culture technology aimed at improving the accuracy of in vitro analyses. Biochem Soc Trans. 2010;38:1072-5.

33. Juuti-Uusitalo K, Klunder L, Sjollema KA, Mackovicova K, Ohgaki R, Hoekstra D, Dekker J, van ljzendoorn SC. Differential effects of TNF (TNFSF2) and IFN-gamma on intestinal epithelial cell morphogenesis and barrier function in three-dimensional culture. PLoS One. 2011;6:e22967.

34. Elamin E, Masclee A, Troost F, Dekker J, Jonkers D. Activation of the epithelial-to-mesenchymal transition factor snail mediates acetaldehyde-induced intestinal epithelial barrier disruption. Alcohol Clin Exp Res. 2014;38:344-53.

35. Elamin E, Masclee A, Dekker J, Jonkers D. Ethanol disrupts intestinal epithelial tight junction integrity through intracellular calcium-mediated Rho/ROCK activation. Am J Physiol. 2014;306:G677-85.

36. Wilcz-Villega EM, McClean S, O'Sullivan MA. Mast cell tryptase reduces junctional adhesion molecule-A (JAM-A) expression in intestinal epithelial cells: implications for the mechanisms of barrier dysfunction in irritable bowel syndrome. Am J Gastroenterol. 2013;108:1140-51.

37. Taira M TJ, Kondo M, Kawatani K, Nagai A. Serum B12 tryptase level as a marker of allergic airway inflammation in asthma. J Asthma. 2002;39:315-22.

38. Hanson PJ Moran AP, Butler K. Paracellular permeability is increased by basal lipopolysaccharide in a primary culture of colonic epithelial cells; an effect prevented by an activator of Toll-like receptor- 2 . Innate Immun. 2011;17:269-82. 
39. Guo S, Al-Sadi R, Said HM, Ma TY. Lipopolysaccharide causes an increase in intestinal tight junction permeability in vitro and in vivo by inducing enterocyte membrane expression and localization of TLR-4 and CD14. Am J Pathol. 2013;182:375-87.

40. Pastor Rojo O, Lopez San Roman A, Albeniz Arbizu E, de la Hera Martinez A, Ripoll Sevillano E, Albillos Martinez A. Serum lipopolysaccharide-binding protein in endotoxemic patients with inflammatory bowel disease. Inflamm Bowel Dis. 2007;13:269-77.

41. O'Dwyer ST, Michie HR, Ziegler TR, Revhaug A, Smith RJ, Wilmore DW. A single dose of endotoxin increases intestinal permeability in healthy humans. Arch Surg. 1988;123:1459-64.

42. Keszthelyi D, Masclee AA. Effects of proton pump inhibitor therapy in the distal gut: putting the pieces together. Dig Dis Sci. 2012;57:2487-9.

43. Keszthelyi D, Dackus GH, Masclee GM, Kruimel JW, Masclee AA. Increased proton pump inhibitor and NSAID exposure in irritable bowel syndrome: results from a case-control study. BMC Gastroenterol. 2012;12:121.

44. Ohmori T, Yang JL, Price JO, Arteaga CL. Blockade of tumor cell transforming growth factor-betas enhances cell cycle progression and sensitizes human breast carcinoma cells to cytotoxic chemotherapy. Exp Cell Res. 1998;245:350-9.

45. Kim JB. Three-dimensional tissue culture models in cancer biology. Semin Cancer Biol. 2005;15:365-77. 


\section{Chapter 7}

Randomized clinical trial on the effect of a multispecies probiotic on visceroperception in hypersensitive IBS patients

S Ludidi, DM Jonkers, CJ Koning, JW Kruimel, L Mulder, I Besseling-van der Vaart, JM Conchillo, AAM Masclee

Neurogastroenterol Motil. 2014;26:705-714 


\section{Abstract}

\section{Background}

Irritable bowel syndrome (IBS) is characterized by heterogeneous pathophysiology and low response-to-treatment. Up to $60 \%$ of IBS patients suffers from visceral hypersensitivity, which is associated with symptom severity and underlying pathophysiological mechanisms. Recently, positive effects of probiotics in IBS have been reported, but overall the response was modest. We performed a study in IBS patients, characterized by visceral hypersensitivity measured with the rectal barostat, aiming to assess the effect of six weeks of multispecies probiotic mix on visceral pain perception.

\section{Methods}

We conducted a randomized, placebo-controlled, double blind trial in forty Rome III IBS patients with visceral hypersensitivity. Prior to intake, patients kept a two-week symptom diary and underwent a rectal barostat measurement. When hypersensitivity was confirmed, participation was allowed and patients received a multispecies probiotic with in vitro proven potential beneficial effects on mechanisms contributing to visceral hypersensitivity ( 6 different probiotic strains; $10^{9} \mathrm{cfu} / \mathrm{gr}$ ), or a placebo product of 1 sachet (5gr) per day for 6 weeks. At the end of the intervention period visceroperception and symptoms were re-assessed.

\section{Results}

Thirty-five patients completed the trial. The percentage of patients with visceral hypersensitivity decreased significantly in the probiotic and placebo group $(76.5 \%$ and $71.4 \%$, respectively; N.S. between groups). Improvement in pain scores and mean symptom score (MSS) did not differ between the probiotic and placebo group.

\section{Conclusion}

In this placebo-controlled trial in IBS patients with visceral hypersensitivity, no significant effect of a multispecies probiotic on viscerperception was observed. 


\section{Introduction}

Irritable bowel syndrome (IBS) is a heterogeneous functional disorder associated with changes in bowel habits and abdominal discomfort or pain. Its prevalence is estimated to be around $10-15 \%{ }^{1,2}$. The pathophysiology of IBS is multifactorial and recent publications have focused on alterations in intestinal microbiota composition, visceral sensitivity, and intestinal permeability ${ }^{3-8}$.

Therapeutic interventions are mainly directed towards symptom reduction with overall low efficacy ${ }^{9}$. Interventions that focus on subgroups of IBS patients that share a specific pathophysiological characteristic, may be an alternative therapeutic approach that has not been explored ${ }^{10}$.

Visceral hypersensitivity is an important hallmark of IBS and is present in up to $60 \%$ of IBS patients. In clinical research, visceroperception is assessed under stimulated conditions with mechanical distension using barostat to provoke sensations of discomfort or visceral pain in a standardized fashion. Distensions applied to the rectum using the barostat, can induce visceral sensations, typical for $\mathrm{IBS}^{11}$. Furthermore this increased rectal sensitivity has shown to be related to symptom severity in daily life ${ }^{12,13}$.

Previous studies found that the interplay between an increased intestinal permeability and inflammation may contribute to visceral hypersensitivity ${ }^{14}$. Increased paracellular permeability has been reported in up to $40 \%$ of IBS patients ${ }^{15}$ and may lead to increased translocation of bacteria and their products, inducing hypersensitivity by targeting the enteric neurons ${ }^{15}$. In addition, increased release of immunologic mediators e.g. histamine and cytokines has been reported in (subgroups of) IBS, via sensitization of neuronal cells, resulting in visceral hypersensitivity ${ }^{3,16-20}$.

Probiotics have the potential to influence the intestinal microbiota and they may affect intestinal barrier function and/ or have anti-inflammatory potential. Recently Agostini et al. have shown that Bifidobacterium lactis CNCM I-2494 reduces visceral hypersensitivity by normalizing intestinal barrier function in rats ${ }^{21}$. Furthermore, a study by Rousseaux et al. demonstrated that Lactobacillus acidophilus NCFM has analgesic properties by inducing the expression of both $\mu$-opioid and cannabinoid receptors in intestinal epithelial cells ${ }^{22}$. These findings indicate that probiotics may have the potential to affect viscerosensation directly or indirectly.

Previous studies with probiotic products have primarily focused on daily symptoms in IBS patients. A recent meta-analysis by Hoveyda et al., pointed to a small but significant improvement in overall symptom scores (both continuous and dichotomous data) after probiotic intake, with evidence based on six randomized controlled trials ${ }^{10}$. However, 
overall beneficial results of probiotic interventions in IBS were modest and also negative outcomes have been documented ${ }^{23,24}$. Methodological limitations, different bacterial strains and differences in treatment dosages and/ or duration are likely to have affected these study outcomes ${ }^{25}$. In addition, heterogeneous groups of IBS patients have been included in these studies, rather than a selection of IBS patients based on pathophysiological characteristics. None of the studies up to now has chosen an approach by evaluating effects of probiotics targeting on one common pathophysiological mechanism.

We hypothesized that implementing a therapeutic intervention in IBS patients with documented visceral hypersensitivity, in which the intervention is known to affect mechanistic factors that contribute to visceral hypersensitivity, has the potential to reduce visceral hypersensitivity (primary aim). Focusing on a well-defined subgroup of IBS patients with visceral hypersensitivity may improve therapeutic efficacy. Since visceral hypersensitivity in IBS is related to symptom severity ${ }^{12,13}$, the secondary aim was to explore the effect of this probiotic product on IBS symptoms. As several putative mechanisms contribute to visceral hypersensitivity, we used not a single probiotic strain but a combination of strains with properties targeting several of these mechanisms.

\section{Materials and methods}

\section{Participants}

Between January 2008 and September 2011 eligible patients were recruited via the outpatient clinic of the division Gastroenterology-Hepatology of the Maastricht University Medical Center+ as well as via local advertisements. Prior to participation, medical history was taken.

Patients were allowed to participate in the study if they were between 18-65 years of age with a body mass index (BMI) between $18.5-30 \mathrm{~kg} / \mathrm{m}^{2}$. All patients complied with the diagnosis of IBS according to Rome III criteria. Major exclusion criteria comprised the use of pre- and/ or probiotics one month or less prior to participation and the use of anti-depressants and/ or antibiotics three months or less prior to participation, and/or alcohol intake over 15 consumptions per week. Furthermore, patients were excluded if they had undergone major abdominal surgery.

All patients gave written informed consent. The study protocol had been approved by the Maastricht University Medical Centre+ Committee of Ethics (reference number MEC 08-1-031) and was executed according to the Declaration of Helsinki (59 ${ }^{\text {th }}$ general 
assembly of the WMA, Seoul, South Korea, Oct. 2008). The study has been registered in the US National Library of Medicine (http://www.clinicaltrials.gov, NCT00702026).

\section{Study design}

The study was executed according to a randomized, placebo-controlled, double-blind design. The total duration of the intervention period was 42 days. Patients were selected for participation based on their previous barostat measurement. At baseline, patients underwent a barostat measurement to confirm visceral hypersensitivity. For a period of 6 weeks, patients consumed one sachet containing either $5 \mathrm{~g}$ of a multispecies probiotic or $5 \mathrm{~g}$ placebo once daily. A final barostat measurement was performed at the last day of the intervention. Two weeks prior to baseline barostat and the last two weeks of the intervention, patients kept a 14-day diary for assessment of symptoms and stool pattern, according to the Bristol stool form scale ${ }^{26}$. Patients were informed that the use of pre- and/ or probiotics or antibiotics during the trial was strictly prohibited.

\section{Multispecies probiotic}

The multispecies probiotic (Winclove 801) was kindly supplied by Winclove b.v., Amsterdam, the Netherlands and contained 6 bacterial species (Bifidobacterium lactis W52, Lactobacillus casei W56, Lactobacillus salivarius W57, Lactococcus lactis W58, Lactobacillus acidophilus NCFM and Lactobacillus rhamnosus W71) at $10^{9} \mathrm{cfu} / \mathrm{gr}$, with maize starch, maltodextrins and mineral mix. The strains were selected based on their resistance to gastric acid, digestive enzymes and bile toxicity and their in vitro potential to beneficially affect parameters associated with visceral hypersensitivity, i.e. inhibition of pro-inflammatory cytokines ${ }^{27-29}$. Furthermore, L. acidophilus NCFM has previously shown to exert analgesic functions in the gut of Sprague-Dawley rats and to induce expression of the $\mu$-opioid and cannabinoid receptor, hereby potentially affecting nociceptive pathways ${ }^{22}$.

The placebo was indistinguishable from the probiotic product in color, smell and taste, but contained no bacteria. All sachets were blinded and coded and block-wise randomization was performed centrally by Winclove b.v. Participants, principal investigator and all other personnel directly involved in the study, were blinded to treatment allocation. Each participant consumed one sachet daily (before breakfast) containing $5 \mathrm{~g}$ probiotic or placebo, for 6 weeks. Sachets had to be dissolved in a glass of lukewarm water, left for $10 \mathrm{~min}$, stirred, and thereafter ingested. 


\section{Barostat measurement}

Participants arrived in the hospital after an overnight fast. They received a rectal enema containing at least $60 \mathrm{~m}$ of tap water to clean the rectum and were instructed to void rectal contents five minutes thereafter.

Rectal perception was measured with an electronic barostat (Distender II; G\&J Electronics, Toronto, ON, Canada, part: C7-CB-R) as described previously ${ }^{30}$, using a balloon of non-compliant material (Mui Scientific, Missisauga, ON, Canada, part: C7-2CB-R), lubricated with KY-gel (Johnsson \& Joshnsson, Longhorne, PA, USA). The barostat protocol consisted of three subsequent procedures for the assessment of visceral perception, starting with I. a First distension at $25 \mathrm{mmHg}$ to unfold the bag; II. measurement of the minimal distension pressure (MDP) and III. assessment of visceral perception of pain, urge and discomfort on a visual analogue scale (VAS) between 0 and $100 \mathrm{~mm}$, according to a semi-random staircase protocol between $0-50 \mathrm{mmHg}$ above $\mathrm{MDP}^{30}$.

\section{Primary parameter: visceral perception}

Treatment efficacy was evaluated by assessment of visceral hypersensitivity before and after the intervention. Responders to treatment showed absence of visceral hypersensitivity after the intervention. Visceral hypersensitivity was calculated based on the perception of pain. A previous study by our group defined the cut-off for visceral hypersensitivity based on the mean pain threshold found in healthy controls minus 2SD. This resulted in a VAS-score $>10 \mathrm{~mm}$ before or at pressure step $23 \mathrm{mmHg}$ to be the curt-off for visceral hypersensitivity ${ }^{13}$. In the present study, the same cut-off was used to define visceral hypersensitivity. Note that at inclusion only patients that were hypersensitive to rectal distensions were allowed to proceed with the study.

Next, the perception data for rectal distensions between $0-50 \mathrm{mmHg}$ were analyzed for intensity of pain, as well as urge and discomfort using VAS-scoring between 0-100 mm. Finally, thresholds for pain, urge and discomfort and overall first sensation were assessed $^{31}$.

\section{Secondary parameters: symptom scores}

Two weeks prior to participation and in the final 2 weeks of supplementation, patients kept a symptom diary in which they scored seven symptoms on a 5-point Likert-scale (i.e. flatulence, bloating, abdominal cramping, abdominal pain, nausea, stools with blood and stools with mucus). The number of symptom free days over a period of 2 weeks and the mean symptom composite score (MSS) were calculated, based on the individual symptoms. Fecal consistency and frequency were scored according to the 
Bristol Stool Form (26) and furthermore response to therapy was calculated. A $30 \%$ or greater improvement in symptom scores was regarded as clinically relevant.

\section{Statistics}

Treatment allocation was concealed to all investigators and participants, until the study had been completed and all analyses had been performed.

Sample size was determined for the primary outcome of the study, i.e. pain perception, using an alpha $=0.05$ and a beta $=20$ (power $=80 \%$ ). Based on previous work ${ }^{13}$, in which we reported a mean pain threshold of $22.2 \pm 9.1 \mathrm{mmHg}$ (mean \pm SD) in IBS patients and considering an increase of $7 \mathrm{mmHg}$ clinically relevant, 17 patients per group were required to reach statistical significance. Although the study was not powered to reach any significant effect on symptoms, self-reported symptom scores were included as secondary outcomes.

Urge, discomfort and pain curves were analyzed using a Gaussian non-linear regression, including a random effect and an autocorrelation ${ }^{32}$. The effect of treatment or placebo on urge, discomfort and pain was considered significant if the $90 \%$ confidence intervals (Cls) did not overlap ${ }^{30,31,33}$. Furthermore, deltas of area under curves (AUCs) after vs. before intervention were compared between probiotics and placebo to assess and quantify the effect of the intervention between groups. Data was tested for normality using the Kolmogorov-Smirnov test. Age and BMI were analyzed parametrically, perception thresholds, and symptoms were compared non-parametrically, using a Mann-Whitney $U$ test and a Wilcoxon signed rank test to compare data between and within groups, respectively. Dichotomous variables were compared using a Chi-square test. A two-sided P-level of 0.05 was considered to be statistically significant. All data were analyzed using SPSS 18.0 for Macintosh, Chicago IL. All data were analyzed by intention-to-treat (ITT) and per protocol (PP) analyses.

\section{Results}

In total, 40 patients were included in the ITT analyses ( 21 in probiotic and 19 in placebo group). Five patients did not complete the study for several reasons (too time consuming, loss of interest, invasiveness of barostat), resulting in 35 patients that were included in the PP analyses (19 in probiotic and 16 in placebo group; Figure 7.1). The outcomes by PP analyses did not differ from ITT analyses. 


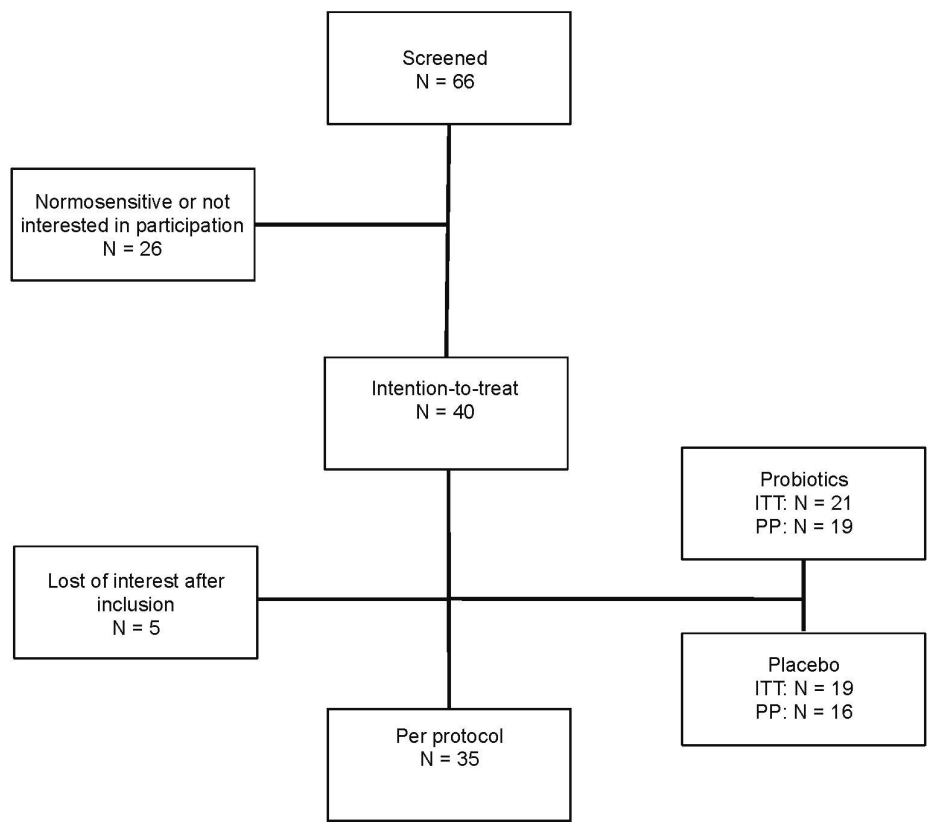

Figure 7.1 Flow-chart of IBS patients that participated in the trial. Graph adjusted.

\section{Patient characteristics}

In Table 7.1, the patient characteristics are presented for the probiotic and the placebo group included in the ITT analysis. Mean age $( \pm$ SEM) did not differ between the probiotic and placebo group $(P=0.93)$, nor did the distribution of sexes $(P=0.74)$, BMI $(P=0.79)$, predominant bowel habits $(P=0.51)$ and disease duration $(P=0.80)$.

Table 7.1 Patient characteristics in probiotic and placebo group included in the ITT analysis (BMI=body mass index; IBS-C=constipation-predominant IBS; IBS-D=diarrhoea-predominant IBS; IBS-M=IBS with mixed stools; IBS-U=unspecified IBS).

\begin{tabular}{lccc}
\hline Parameter & Probiotic $(\mathrm{N}=21)$ & Placebo $(\mathrm{N}=19)$ & $P$-value \\
\hline Age (Mean \pm SEM) & $40.0 \pm 2.2 \mathrm{yr}$ & $41.1 \pm 4.2 \mathrm{yr}$ & 0.93 \\
Sex (male: female) & $6: 15$ & $7: 12$ & 0.74 \\
BMI (Mean \pm SEM) & $25.8 \pm 1.1 \mathrm{~kg} / \mathrm{m}^{2}$ & $25.1 \pm 0.9 \mathrm{~kg} / \mathrm{m}^{2}$ & 0.79 \\
IBS-type [-C; -D; -M; -U] (\%) & $5 ; 43 ; 33 ; 19$ & $17 ; 44 ; 28 ; 11$ & 0.51 \\
Disease duration (Mean \pm SEM) & $3.27 \pm 1.34 \mathrm{yr}$ & $2.73 \pm 1.97 \mathrm{yr}$ & 0.80 \\
\hline
\end{tabular}




\section{Primary parameter: visceral perception}

Patients were selected based on the presence of visceral hypersensitivity. At baseline, all included patients showed visceral hypersensitivity. After 6 weeks of intervention, the percentage of hypersensitive patients decreased significantly within both the probiotic and placebo group (towards $76.5 \%$ and $71.4 \%$, respectively; both $P<0.05$ versus baseline). However, response rates did not differ between the groups $(P=0.24)$.

\section{Scores for pain, discomfort and urge}

The delta values (after versus before intervention) of the area under the curve (in arbitrary units) for pain did not significantly differ between the probiotic versus placebo group (248.6 [115.3-383.0] vs. 253.5 [126.5-382.5], respectively). Following from the non-linear regression analysis, scores for pain significantly decreased in both the probiotic and placebo group (Figure 7.2).

Neither for urge, nor for discomfort did the delta values of the AUCs significantly differ between the probiotic and placebo group. As for pain, scores for urge and discomfort decreased significantly within groups (data not shown).
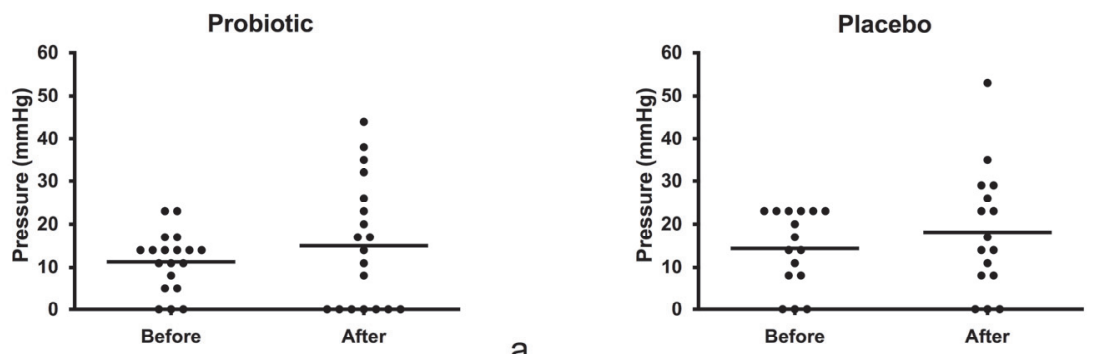

b
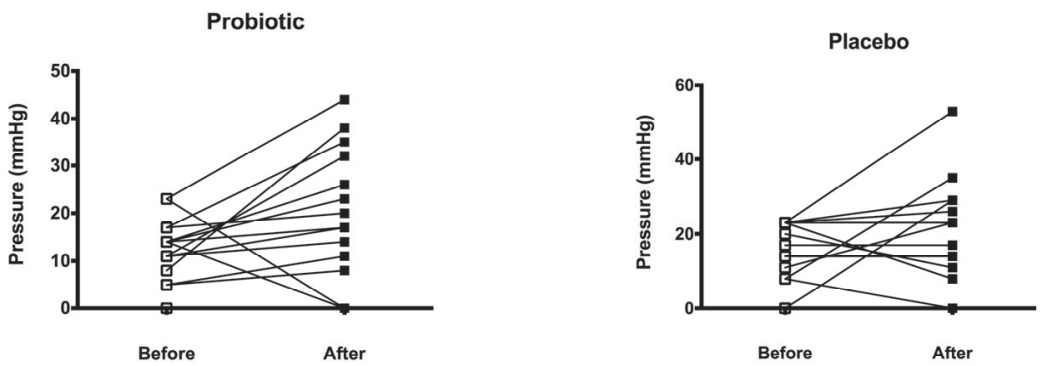

C

Figure 7.2 Mean pain VAS-scores \pm SEM in probiotic (a) and placebo (b) group before (open squares) and after (black squares) intervention (ITT; $\mathrm{N}=21$ and 19 in probiotic and placebo group, respectively) 


\section{Thresholds for pain, urge and discomfort}

Delta values for the thresholds of pain, urge and discomfort neither differed significantly between the probiotic and placebo group (data not shown). Figure 7.3 shows that the median thresholds for pain did not differ significantly before versus after intervention within the probiotic nor the placebo group. This holds also true for urge and discomfort (Figure 7.4). Finally, the difference in first sensation to rectal distension was not statistically significant between probiotic vs. placebo $(0.00$ $[-0.17-15.00]$ vs. $0.00[-17.00-23.00] ; P=0.78)$.

Probiotic

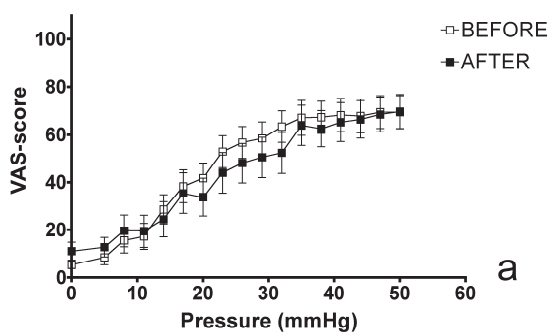

Placebo

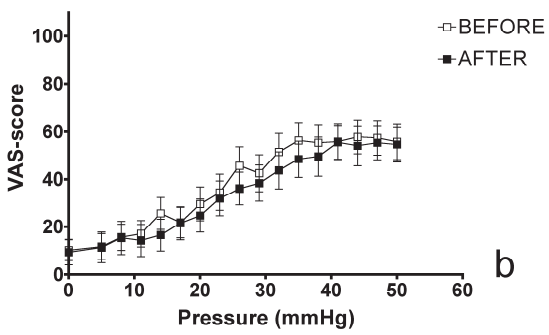

Figure 7.3 Pain perception scores in probiotic (a) and placebo (b) group before (open squares) and after (black squares) intervention (PP; $\mathrm{N}=19$ and 16 in probiotic and placebo group, respectively). Graph adjusted.

\section{Secondary parameters: symptoms}

None of the baseline scores for IBS-symptoms showed a significant difference between the probiotic versus the placebo group. Table 7.2 shows the results for IBS-symptoms at baseline versus after the intervention, according to the ITT analysis.

Delta values (after versus before) for MSS were not significantly different between the probiotic and the placebo group (-0.66 [-3.77-1.64 vs. $-1.36-[-7.42-1.43])$. In the probiotic group MSS did not significantly decrease after the intervention (7.82 [3.21-20.23 vs. 6.36 [1.72-16.75]; $P=0.12$ ). However, the placebo group did show a statistically significant decrease of mean composite symptom score, MSS: 6.33 [2.93-16.00] vs. -4.74 [0.85-15.43]; $P<0.01)$.

With regards to individual symptoms, median delta values (after versus before) were not statistically different between probiotic and placebo, with the exception of flatulence, pointing to a larger improvement in the placebo compared to the probiotic group $(-0.59[-1.95-0.67]$ vs. $-0.07[-1.03-0.72]) ; P<0.01)$. Within the probiotic group, individual symptoms did not significantly decrease, whereas the symptoms flatulence, 
bloating and abdominal cramping reduced significantly (all $P<0.01$ ) in the placebo group. Abdominal pain did not change in both groups.

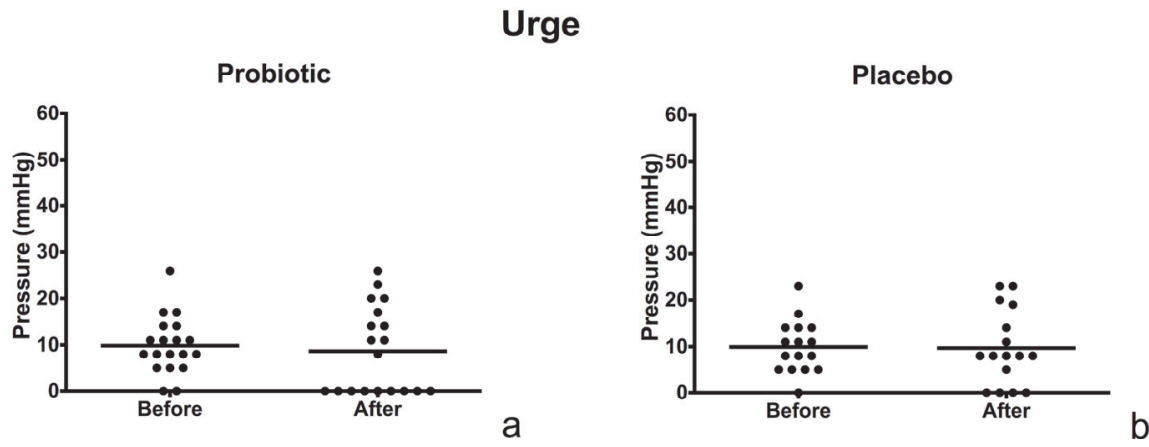

\section{Discomfort}
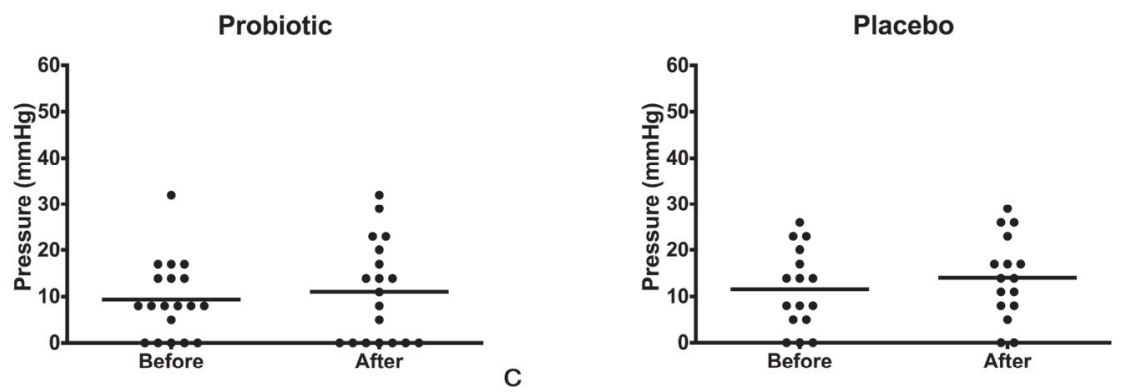

Figure 7.4 Thresholds for urge and discomfort probiotic $(a, c)$ and placebo $(b, d)$ group, respectively before and after intervention (PP; N = 19 and 16 in probiotic and placebo group, respectively).

The difference in number of symptom-free days (delta values after versus before) was not statistically significant between the probiotic and placebo group. Within groups, the placebo group showed a small, though statistically significant increase in symptom-free days, whereas no such effect was observed in the probiotic group (Table 7.2).

Finally, in none of the groups did any of the symptoms correlate with intensity of visceroperception, neither at baseline, nor at the end of the intervention.

\section{Mean symptom improvement}

Based on a $>30 \%$ improvement in MSS, $19 \%$ in the probiotic group and $39 \%$ in the placebo group showed symptom improvement. This was not significantly different between groups $(P=0.18)$. Overall, when combining the probiotic and placebo group, 
patient characteristics did not differ between the patients that did and did not show improvement with regards to mean age (39.3 \pm 14.2 vs. $38.8 \pm 14.0(P=0.94)) ; B M I$ $(24.9 \pm 2.5$ vs. $26.0 \pm 5.5(P=0.62))$ and female $\operatorname{sex}(75 \%$ vs. $61.1 \%(P=0.67))$.

Table 7.2 IBS-related symptoms expressed as median [range] before and after intervention in probiotic $(\mathrm{N}=21)$ and placebo group $(\mathrm{N}=19)$ (ITT). Abbreviations $A b$. and $\mathrm{SF}$ refer to abdominal and symptom-free, respectively.

\begin{tabular}{lcc}
\hline & Before - Median [range] & After - Median [range] \\
\hline MSS probiotic & $7.82[3.21-20.23]$ & $6.36[1.72-16.75]$ \\
MSS placebo & $6.33[2.93-16.00]$ & $4.74[0.85-15.43]^{* *}$ \\
Flatulence probiotic & $1.64[0.07-3.21]$ & $1.43[0.10-3.43]^{* *}$ \\
Flatulence placebo & $1.57[0.42-3.00]$ & $1.00[0.00-2.79]^{*}$ \\
Bloating probiotic & $1.79[0.00-3.79]$ & $1.29[0.07-3.73]^{*}$ \\
Bloating placebo & $1.57[0.00-3.71]$ & $1.21[0.00-3.23]^{*}$ \\
Ab. cramping probiotic & $1.62[0.14-3.21]$ & $1.29[0.00-3.18]^{*}$ \\
Ab. cramping placebo & $1.18[0.14-4.00]$ & $0.58[0.00-3.07]^{*}$ \\
Ab. pain probiotic & $1.71[0.07-3.21]$ & $1.69[0.14-3.64]$ \\
Ab. pain placebo & $1.62[0.00-3.54]$ & $0.91[0.00-3.23]$ \\
SF-days probiotic & $<0.01[0.00-9.00]$ & $<0.01[0.00-9.00]$ \\
SF- days placebo & $<0.01[0.00-7.00]$ & $<0.01[0.00-13.00]^{*}$ \\
\hline
\end{tabular}

* $P<0.05,{ }^{* *} P<0.01$

\section{Gender differences}

We observed gender-related response differences in the placebo group, implicating that MSS, bloating and abdominal cramping as well as symptom-free days only improved in the female subjects, but not in the male subjects. In the probiotic group, no gender differences were observed. Table 7.3 summarizes median symptom improvement of female and male subjects in both groups.

Table 7.3 Symptom improvement versus baseline (median [range]), in probiotic and placebo group (ITT), split for sex. Abbreviations Ab. and SF refer to abdominal and symptom-free, respectively. Total number of female patients in the probiotic and placebo group was 15 and 12 , respectively.

\begin{tabular}{lcc}
\hline Symptom improvement & Female (N=27) & Male (N=13) \\
\hline MSS probiotic & $-0.06[-3.77-1.64]$ & $-1.18[-1.80--0.25]$ \\
MSS placebo & $-1.49[-7.42--0.32]^{*}$ & $-0.93[-3.87-1.43]$ \\
Flatulence probiotic & $-0.16[-0.77-0.72]$ & $-0.36[-1.03-0.67]$ \\
Flatulence placebo & $-0.62[-1.95-0.67]$ & $-0.27[-1.80-0.00]^{*}$ \\
Bloating probiotic & $-0.36[-1.09-0.86]$ & $-0.06[-1.70-1.50]$ \\
Bloating placebo & $-0.43[-1.64--0.10]^{*}$ & $-0.43[-1.85-0.50]$ \\
Ab. cramping probiotic & $-0.52[-0.93-0.97]$ & $-0.66[-0.76-0.45]$ \\
Ab. cramping placebo & $-0.30[-1.86-0.00]^{*}$ & $-0.21[-1.07-0.18]$ \\
Ab. pain probiotic & $-0.13[-0.79-1.50]$ & $0.39[-0.29-0.67]$ \\
Ab. pain placebo & $-0.22[-1.60-0.30]$ & $-0.14[-0.36-2.02]$ \\
SF-days probiotic & $0.00[-2.00-8.00]$ & $0.00[-1.00-3.00]$ \\
SF- days placebo & $2.00[0.00-6.00]^{*}$ & $0.00[0.00-11.00]$ \\
\hline
\end{tabular}
${ }^{*} P<0.05$ 


\section{Discussion}

In the present study, no significant difference in effect of the multispecies probiotic product versus placebo was observed on visceroperception in a group of IBS patients characterized by visceral hypersensitivity. When analyzing data within groups, not only in the probiotic group but also in the placebo group a significant reduction was observed in pain scores during mechanical rectal distensions.

IBS is a complex heterogeneous condition of multifactorial origin. Previous intervention studies assessing the effect of probiotics on symptoms have shown (modest) beneficial but also negative results. Comparing results of various studies is difficult due to methodological shortcomings such as differences in study design, type of probiotic used, and outcome parameters. In addition, previous studies often used non-validated and subjective endpoints and/ or included heterogeneous patient populations.

Most probiotic intervention studies in IBS have focused on daily symptoms ${ }^{10,34}$. We have chosen for a distinct approach i.e. we assessed the effect of the intervention on visceroperception measured by rectal barostat ${ }^{35}$. IBS patients were specifically selected for the study based on the presence of visceral hypersensitivity.

Previously, it has been postulated that increased intestinal permeability and inflammation and immune response relate to visceral hypersensitivity ${ }^{14}$. From this perspective, hypersensitive IBS patients were ought to benefit from a probiotic intervention that modulates the abovementioned factors involved in the pathophysiology of visceral hypersensitivity.

Nonetheless, we did not observe any beneficial effect of the probiotic over the placebo group. After six weeks of intervention, a significant decrease in visceral pain perception was observed in the probiotic group and the placebo group. We know that an increased intensity of visceral symptoms, i.e. pain, is present in about two-third of the overall IBS population when using the barostat procedure. It is well known that in IBS, symptoms decline over time and prevalence is higher among younger vs. older patients ${ }^{36}$. IBS patients tend to seek medical care when their symptoms are most pronounced. IBS symptoms vary considerably with a tendency to decrease after a maximum has been reached. So, when including IBS patients with high perception scores, they are prone to show improvement over time regardless of the intervention (active or placebo), as our data indicate. In addition, several clinical trials have reported on the magnitude of the placebo response in IBS patients, which may vary between $30 \%$ and $50 \%{ }^{37-39}$.

With respect to the secondary parameters: At baseline, the placebo and probiotic group were comparable regarding baseline characteristics, including symptom scores 
and disease duration. Symptom scores did not improve significantly in the probiotic group, but the placebo group showed significant improvements in the number of symptom free days, MSS as well as on the individual symptoms flatulence, bloating and abdominal cramps.

It should be taken into account that the study was not powered to reach statistical significances for the secondary parameters. Nonetheless, when analyzing symptom scores in more detail, it became apparent that the observed beneficial effect in the placebo group results from a significant symptom reduction among female and not among male patients. It remains unclear why female patients respond differently than male patients.

Although a placebo-effect has been observed in IBS patients previously ${ }^{37,38}$, the probiotic group in our study did not show an improvement in symptom scores. Thus, the probiotic product potentially masked or impaired the placebo-effect in these patients. The placebo group after all did show improvement with respect to several symptoms. Form another perspective, at least symptoms did not worsen in the probiotic group and it is noteworthy that the individual symptoms that improved in the placebo group (i.e. flatulence, bloating and abdominal cramping) all can be linked to presence of excessive gas or gas production. Previous studies have shown that increased numbers of Bifidobacterium spp. may increase flatulence ${ }^{23,40,41}$. We assume that the presence of some strains in the probiotic mixture may have contributed to an increased gas production and thereby to an increase in gas-related symptoms. Hence, this could explain why the symptoms flatulence, but also bloating and abdominal cramping improved in the placebo group, but not in the probiotic group.

Several limitations of the study should be mentioned. First, the duration of the intervention: the supplementation period we implemented was 6 weeks. This was longer than the lower limits of duration generally applied in other probiotic studies ${ }^{42}$. Several groups have reported positive effects on symptoms after a four-week supplementation period ${ }^{43-46}$. On the contrary however, other groups have shown negative effects after an equally long supplementation period of 4 weeks ${ }^{47,48}$. More pronounced improvements have been found in trials where probiotic supplementation lasted for at least 8 weeks ${ }^{23}$. Since IBS is a chronic condition it may require longer term or even continuous supplementation of probiotics to result in significant alterations at symptom level.

Second, an important point to be taken into account is the composition of the probiotic product. Studies with probiotics that have demonstrated symptom improvement in IBS patients, in general assessed the effect of only a single bacterial strain. A recent review 
concluded that Bifidobacterium infantis 35624 at a dose of $1 \times 10^{8} \mathrm{cfu} /$ day taken for at least 4 weeks $^{44}$, showed the strongest evidence for improving symptoms in IBS ${ }^{23,42,44}$. The present study used a mix of six different bacterial strains specifically selected for their individual potential to improve symptoms. L. acidophilus NCFM was selected based on its antinociceptive properties ${ }^{22}$, whereas other strains (e.g. Lactobacillus salivarius, Lactobacillus casei) were selected by their in vitro potential to improve epithelial integrity and inflammatory status ${ }^{27,29}$, which may contribute to improved visceral perception. Although multispecies and multistrain probiotics may potentially have higher treatment efficacy compared to monostrain probiotics ${ }^{49,50}$, it cannot be excluded that competition occurs between ingested species or strains. Consequently, negative effects on outcome due to bacterial interactions may occur.

Third, number of patients included in the trial was probably low to detect large differences in treatment efficacy between both groups. Although this study was executed as randomized placebo-controlled double-blind study and powered at 34 hypersensitive patients to detect a significant improvement in probiotic vs. placebo, this may have been too low to demonstrate clear differences. Nonetheless, drop-out rate was low ( 5 in total, of which 2 in probiotic and 3 in placebo) and the per protocol analysis did not differ from the intention-to-treat analyses. Furthermore, it is important to note that the study was not powered to detect significant differences in selfreported symptom scores as measured by the symptom diary.

While we considered the strategy for an IBS treatment aiming at patients that share a common clinical hallmark an attractive and alternative approach, we were not able to demonstrate any improvement in visceral sensitivity during probiotics vs. placebo. It is important to note that theoretical assumptions of probiotics that are based on in vitro work should be considered with care and require validation in vivo.

We cannot exclude that the mix of probiotics may have resulted in overall inhibitory and thereby negative effects on various parameters and mechanisms in vivo. The outcome of our study may have been influenced by the duration of supplementation as well as the composition of multispecies probiotic product. However, the concept of therapeutic interventions in targeted subgroups of IBS patients with a common pathophysiological characteristic deserves further evaluation.

In this placebo controlled trial in IBS patients with visceral hypersensitivity, no significant effect of a multispecies probiotic on visceroperception was observed. 


\section{References}

1. Talley NJ. Irritable bowel syndrome: definition, diagnosis and epidemiology. Baillieres Best Pract Res Clin Gastroenterol 1999;13:371-84.

2. Hungin AP, Whorwell PJ, Tack J, Mearin F. The prevalence, patterns and impact of irritable bowel syndrome: an international survey of 40,000 subjects. Aliment Pharmacol Ther 2003;17:643-50.

3. Piche T, Barbara G, Aubert P, Bruley des Varannes S, Dainese R, Nano JL, Cremon C, Stanghellini V, De Giorgio R, Galmiche JP, Neunlist M. Impaired intestinal barrier integrity in the colon of patients with irritable bowel syndrome: involvement of soluble mediators. Gut 2009;58:196-201.

4. Camilleri M, Lasch K, Zhou W. The Confluence of Increased Permeability, Inflammation, and Pain in Irritable Bowel Syndrome. Am J Physiol Gastointest Liver Physiol 2012;303:G775-85.

5. Spiller R, Lam C. An Update on Post-infectious Irritable Bowel Syndrome: Role of Genetics, Immune Activation, Serotonin and Altered Microbiome. J Neurogastroenterol Motil 2012;18:258-68.

6. Isolauri E, Salminen S, Ouwehand AC. Microbial-gut interactions in health and disease. Probiotics. Best Pract Res Clin Gastroenterol 2004;18:299-313.

7. Mai V, Braden CR, Heckendorf J, Pironis B, Hirshon JM. Monitoring of stool microbiota in subjects with diarrhea indicates distortions in composition. J Clin Microbiol 2006;44:4550-2.

8. Mertz H, Naliboff B, Munakata J, Niazi N, Mayer EA. Altered rectal perception is a biological marker of patients with irritable bowel syndrome. Gastroenterology 1995;109:40-52.

9. Grundmann O, Yoon SL. Irritable bowel syndrome: epidemiology, diagnosis and treatment: an update for health-care practitioners. J Gastroenterol Hepatol 2010;25:691-9.

10. Hoveyda N, Heneghan C, Mahtani KR, Perera R, Roberts N, Glasziou P. A systematic review and metaanalysis: probiotics in the treatment of irritable bowel syndrome. BMC Gastroenterol 2009;9:15.

11. Nozu T, Okumura T. Visceral sensation and irritable bowel syndrome; with special reference to comparison with functional abdominal pain syndrome. J Gastroenterol Hepatol 2011;26 Suppl 3:122-7.

12. Posserud I, Syrous A, Lindstrom L, Tack J, Abrahamsson H, Simren M. Altered rectal perception in irritable bowel syndrome is associated with symptom severity. Gastroenterology 2007;133:1113-23.

13. van der Veek PP, Van Rood YR, Masclee AA. Symptom severity but not psychopathology predicts visceral hypersensitivity in irritable bowel syndrome. Clin Gastroenterol Hepatol 2008;6:321-8.

14. Zhou Q, Verne GN. New insights into visceral hypersensitivity--clinical implications in IBS. Nat Rev Gastroenterol Hepatol 2011;8:349-55.

15. Zhou Q, Zhang B, Verne GN. Intestinal membrane permeability and hypersensitivity in the irritable bowel syndrome. Pain 2009;146:41-6.

16. Larauche M. Novel insights in the role of peripheral corticotropin-releasing factor and mast cells in stress-induced visceral hypersensitivity. Neurogastroenterol Motil 2012;24:201-5.

17. Binshtok AM, Wang H, Zimmermann K, Amaya F, Vardeh D, Shi L, Brenner GJ, Ji RR, Bean BP, Woolf CJ, Samad TA. Nociceptors are interleukin-1beta sensors. J Neurosci 2008;28:14062-73.

18. Mayer EA, Gebhart GF. Basic and clinical aspects of visceral hyperalgesia. Gastroenterology 1994;107: 271-93.

19. Ait-Belgnaoui A, Bradesi S, Fioramonti J, Theodorou V, Bueno L. Acute stress-induced hypersensitivity to colonic distension depends upon increase in paracellular permeability: role of myosin light chain kinase. Pain 2005;113:141-7.

20. Zhou Q, Souba WW, Croce CM, Verne GN. MicroRNA-29a regulates intestinal membrane permeability in patients with irritable bowel syndrome. Gut 2010;59:775-84.

21. Agostini S, Goubern $M$, Tondereau V, Salvador-Cartier $C$, Bezirard V, Lévèque $M$, Keränen $H$, Theodorou V, Bourdu-Naturel S, Goupil-Feuillerat N, Legrain-Raspaud S, Eutamene H. A marketed fermented dairy product containing Bifidobacterium lactis CNCM I-2494 suppresses gut hypersensitivity and colonic barrier disruption induced by acute stress in rats. Neurogastroenterol Motil 2012;24:376-e172.

22. Rousseaux C, Thuru X, Gelot A, Barnich N, Neut C, Dubuquoy L, Dubuquoy C, Merour E, Geboes K, Chamaillard M, Ouwehand A, Leyer G, Carcano D, Colombel JF, Ardid D, Desreumaux P. Lactobacillus acidophilus modulates intestinal pain and induces opioid and cannabinoid receptors. Nat Med 2007;13:35-7. 
23. Simrén M, Barbara G, Flint HJ, Spiegel BM, Spiller RC, Vanner S, Verdu EF, Whorwell PJ, Zoetendal EG; Rome Foundation Committee. Intestinal microbiota in functional bowel disorders: a Rome foundation report. Gut 2013;62:159-76.

24. Whelan K. Probiotics and prebiotics in the management of irritable bowel syndrome: a review of recent clinical trials and systematic reviews. Curr Opin Clin Nutr Metab Care 2011;14:581-7.

25. Clarke G, Cryan JF, Dinan TG, Quigley EM. Review article: probiotics for the treatment of irritable bowel syndrome--focus on lactic acid bacteria. Aliment Pharmacol Ther 2012;35:403-13.

26. O'Donnell LJ, Virjee J, Heaton KW. Detection of pseudodiarrhoea by simple clinical assessment of intestinal transit rate. BMJ 1990;300:439-40.

27. Timmerman HM, Niers LE, Ridwan BU, Koning CJ, Mulder L, Akkermans LM, Rombouts FM, Rijkers GT. Design of a multispecies probiotic mixture to prevent infectious complications in critically ill patients. Clin Nutr 2007;26:450-9.

28. de Jager W, te Velthuis H, Prakken BJ, Kuis W, Rijkers GT. Simultaneous detection of 15 human cytokines in a single sample of stimulated peripheral blood mononuclear cells. Clin Diagn Lab Immunol 2003;10:133-9.

29. Malago JJ, Tooten PC, Koninkx JF. Anti-inflammatory properties of probiotic bacteria on Salmonellainduced IL-8 synthesis in enterocyte-like Caco-2 cells. Benef Microbes 2010;1:121-30.

30. Ludidi S, Conchillo JM, Keszthelyi D, Koning CJ, Vanhoutvin SA, Lindsey PJ, Leufkens AM, Kruimel JW, Jonkers DM, Masclee AA. Does meal ingestion enhance sensitivity of visceroperception assessment in irritable bowel syndrome? Neurogastroenterol Motil 2012;24:47-53, e3.

31. Ludidi S, Conchillo JM, Keszthelyi D, Van Avesaat M, Kruimel JW, Jonkers DM, Masclee AA. Rectal hypersensitivity as hallmark for irritable bowel syndrome: defining the optimal cutoff. Neurogastroenterol Motil 2012;24:729-33.

32. Lindsey PJ, Kaufmann J. Analysis of a longitudinal ordinal response clinical trial using dynamic models. J R Stat Soc Series B Stat Methodol 2004; 53.

33. Vanhoutvin SA, Troost FJ, Kilkens TO, Lindsey PJ, Hamer HM, Jonkers DM, Venema K, Brummer RJ. The effects of butyrate enemas on visceral perception in healthy volunteers. Neurogastroenterol Motil 2009;21:952-e76.

34. Moayyedi P, Ford AC, Talley NJ, Cremonini F, Foxx-Orenstein AE, Brandt LJ, Quigley EM. The efficacy of probiotics in the treatment of irritable bowel syndrome: a systematic review. Gut 2010;59:325-32.

35. Hammer HF, Phillips SF, Camilleri M, Hanson RB. Rectal tone, distensibility, and perception: reproducibility and response to different distensions. Am J Physiol 1998;274:G584-90.

36. Dunlop SP, Jenkins D, Spiller RC. Age-related decline in rectal mucosal lymphocytes and mast cells. Eur J Gastroenterol Hepatol 2004;16:1011-5.

37. Patel SM, Stason WB, Legedza A, Ock SM, Kaptchuk TJ, Conboy L, Canenguez K, Park JK, Kelly E, Jacobson E, Kerr CE, Lembo AJ. The placebo effect in irritable bowel syndrome trials: a meta-analysis. Neurogastroenterol Motil 2005;17:332-40.

38. Pitz M, Cheang M, Bernstein CN. Defining the predictors of the placebo response in irritable bowel syndrome. Clin Gastroenterol Hepatol 2005;3:237-47.

39. Rogers NJ, Mousa SA. The shortcomings of clinical trials assessing the efficacy of probiotics in irritable bowel syndrome. J Altern Complement Med 2012;18:112-9.

40. Bouhnik Y, Neut C, Raskine L, Michel C, Riottot M, Andrieux C, Guillemot F, Dyard F, Flourié B. Prospective, randomized, parallel-group trial to evaluate the effects of lactulose and polyethylene glycol-4000 on colonic flora in chronic idiopathic constipation. Aliment Pharmacol Ther 2004;19:889-99.

41. Florent C, Flourie B, Leblond A, Rautureau M, Bernier JJ, Rambaud JC. Influence of chronic lactulose ingestion on the colonic metabolism of lactulose in man (an in vivo study). J Clin Invest 1985;75: 608-13.

42. Camilleri M, Mayer EA. Developing irritable bowel syndrome guidelines through meta-analyses: does the emperor really have new clothes? Gastroenterology 2009;137:766-9.

43. Niedzielin K, Kordecki H, Birkenfeld B. A controlled, double-blind, randomized study on the efficacy of Lactobacillus plantarum $299 \mathrm{~V}$ in patients with irritable bowel syndrome. Eur J Gastroenterol Hepatol 2001;13:1143-7. 
44. Whorwell PJ, Altringer L, Morel J, Bond Y, Charbonneau D, O'Mahony L, Kiely B, Shanahan F, Quigley EM. Efficacy of an encapsulated probiotic Bifidobacterium infantis 35624 in women with irritable bowel syndrome. Am J Gastroenterol 2006;101:1581-90.

45. Kim HJ, Vazquez Roque MI, Camilleri M, Stephens D, Burton DD, Baxter K, Thomforde G, Zinsmeister AR. A randomized controlled trial of a probiotic combination VSL\# 3 and placebo in irritable bowel syndrome with bloating. Neurogastroenterol Hepatol 2005;17: 687-96.

46. Zeng J, Li YQ, Zuo XL, Zhen YB, Yang J, Liu CH. Clinical trial: effect of active lactic acid bacteria on mucosal barrier function in patients with diarrhoea-predominant irritable bowel syndrome. Aliment Pharmacol Ther 2008;28:994-1002.

47. Sen S, Mullan MM, Parker TJ, Woolner JT, Tarry SA, Hunter JO. Effect of Lactobacillus plantarum 299v on colonic fermentation and symptoms of irritable bowel syndrome. Dig Dis Sci 2002;47:2615-20.

48. Drouault-Holowacz S, Bieuvelet S, Burckel A, Cazaubiel M, Dray X, Marteau P. A double blind randomized controlled trial of a probiotic combination in 100 patients with irritable bowel syndrome. Gastroenterol Clin Biol 2008;32:147-52.

49. Timmerman HM, Koning CJ, Mulder L, Rombouts FM, Beynen AC. Monostrain, multistrain and multispecies probiotics--A comparison of functionality and efficacy. Int J Food Microbiol 2004;96: 219-33.

50. Chapman CM, Gibson GR, Rowland I. Health benefits of probiotics: are mixtures more effective than single strains? Eur J Nutr 2011;50:1-17. 


\section{Chapter 8}

General discussion 
Chapter 8 


\section{General discussion}

\section{IBS from diversity to subtyping with focus on hypersensitivity}

Although IBS is a highly prevalent functional disorder, the exact pathophysiology remains unclear. The heterogeneous disease phenotype involves several potential pathophysiological mechanisms such as intestinal barrier dysfunction, chronic low grade inflammation, intestinal dysbiosis, disturbed gut-brain interactions and visceral hypersensitivity. Of these factors, visceral hypersensitivity is considered an important hallmark of the disorder, reported in about half of the patients. Visceral perception itself may be affected by several of the above mentioned mechanisms, such as increased intestinal permeability, inflammation, and (enteric) nerve signaling. However, little is known about clinical profiles of patients with or without visceral hypersensitivity and their association with mechanistic factors.

In Maastricht we have taken the initiative to build a cohort of IBS patients for extensive phenotyping, genotyping and genotype-phenotype interactions. We have collected biomaterials such as blood samples, DNA, stools, urine and biopsies and stored them in a biobank. In addition, data on demographics, symptoms, psychological status and patient well-being have been collected. In a subgroup, visceral perception by rectal barostat and intestinal permeability by a multi-sugar test has also been assessed. This strategy will allow us a) to systematically study clinical and psychological profiles, as well as pathophysiological factors, in relation to symptoms, to search for non-invasive markers and b) to develop new therapeutic strategies based either on identical or shared pathophysiological findings or on well-phenotyped subgroups with a common background, for instance with visceral hypersensitivity.

\section{Assessment of visceral perception}

Among the multitude of IBS symptoms, pain has a predominant role. Patients with IBS frequently experience episodes of (recurrent) pain, and abdominal pain is a major constituent of the Rome III criteria for the diagnosis of IBS. IBS patients specifically report visceral/abdominal pain. This pain cannot be traced to a specific organ or anatomical site. Adequate assessment methods are essential in understanding and mapping visceral pain in the patient population of IBS.

The barostat is a widely accepted method to induce and record a symptomatic response either in the rectum or elsewhere in the GI tract, that can subsequently be measured using a Likert or visual analogue scale (VAS). The barostat method has been shown previously, to be able to evoke IBS-specific visceral pain following balloon distensions applied to the colon or to the rectum. In fact, hypersensitivity observed in 
the rectum has been proposed to represent hypersensitivity throughout the colon ${ }^{1}$. In addition, pain perception in response to application of mechanical distensions in IBS patients, was shown to correlate with daily symptoms and with the site of symptom presentation, as indicated by the viscero-somatic referral areas ${ }^{2}$. Compared to symptom diaries or questionnaires, which are prone to recall bias, the barostat measures pain in direct response (i.e. in real time). Nonetheless, it is less representative for the physiological situation as it measures the patient's response at a certain applied stimulus. Another way to measure pain directly related to stimuli, comes from the experience sampling method (ESM). The ESM provides an assessment tool that is more representative for daily practice and for this reason, recent interest has gone to this method. ESM has previously been used in psychology and psychiatry to obtain data on patient's experiences and feelings at 'random' time points during the day. Pilot data within our department suggest that the ESM is promising as it may provide additional data or even new insights in symptom generation and therapy-response in IBS and other disorders.

At present, the rectal barostat is the standard method to assess visceral hypersensitivity in IBS. Using this method, we consistently observed that $50 \%$ to $60 \%$ of our patient population showed visceral hypersensitivity, a finding that is within the range of $30 \%$ and $90 \%$ described previously ${ }^{3,4}$. However, reported prevalences of visceral hypersensitivity in IBS populations vary considerably between GI motility labs, which is partly the result of different protocols used. In order to enhance and optimize the detection of visceral hypersensitivity, we demonstrated in Chapter $\mathbf{2}$ that ingestion of a high-fat liquid meal increased visceral perception of pain, urge and discomfort in patients with IBS. Not only in IBS patients, but also in healthy controls, perception of urge and pain increased postprandially. However, the majority of the IBS patients (86\%) showing postprandial hypersensitivity, were already hypersensitive in the fasted state. As a consequence, the liquid meal did not significantly increase the "sensitivity" of the barostat method to detect visceral hypersensitivity in patients with IBS. In this respect a barostat test without a meal already is sufficient to separate hypersensititive from normosensitive IBS patients.

Further improvement of the barostat procedure in detecting hypersensitivity in IBS patients versus healthy subjects (Chapter 3 ), resulted in a new cut-off value for visceral hypersensitivity, i.e. VAS $\geq 20 \mathrm{~mm}$ at $26 \mathrm{mmHg}$ with $90 \%$ specificity. Based on these criteria, $50 \%$ of our IBS population showed visceral hypersensitivity in response to rectal distensions. Comparable to somatic pain, visceral pain perception can subdivided based on two perception levels: 1 ) by having reduced thresholds to perceive physiological stimuli as being painful (i.e. allodynia) or 2) by an increased perceived intensity (higher pain scores) for pain in response to painful stimuli (i.e. hyperalgesia). In our IBS population (Chapter 4), the majority (almost 90\%) of patients that were 
hypersensitive, showed allodynic responses to rectal distensions, compared to only $10 \%$ in the normosensitive group. Whereas other studies primarily focus on overall pain perception without distinguishing different levels of pain perception, our data clearly demonstrate that apart from increased perception to noxious stimuli, a significant subset of IBS patients exhibits early visceral pain perception, with lower thresholds to perceive pain. Distinguishing between these levels of altered pain perception may help to identify potential mechanistic factors or pathways that are involved in the pathophysiology of IBS.

\section{Characterisation of hypersensitive IBS patients}

In order to characterize hypersensitive IBS patients from a more clinical perspective, IBS patients with and without visceral hypersensitivity were compared (Chapter 4). Most studies have primarily focused on the different clinical IBS-subtypes, while data on demographics, symptom predominance and psychological factors of patients with hypersensitivity are limited. In our approach, we aimed to identify patients with IBS based on the presence of a mechanistic factor that is visceral hypersensitivity.

Comparing the hypersensitive with the normosensitive patients, analysis of these characteristics did reveal some differences. IBS symptoms and also symptoms rated by the GSRS were significantly increased in patients with visceral hypersensitivity versus those without. Apart from an increased severity of gastrointestinal symptoms (e.g. abdominal pain, perceived intensity of symptoms) visceral hypersensitivity was associated with female sex, younger age and use of selective serotonin reuptake inhibitor (SSRI) medication. In general the IBS population is characterized by female predominance. Gender differences have previously been also described with respect to pain sensation and generally, women have lower thresholds for pain compared to men. This has been attributed to influences of sex hormones and differences in in CNS pain processing $^{5,6}$.

The enteric nervous system plays an important role in sensitizing (painful) stimuli. These stimuli are further processed in the central nervous system (CNS). The gut-brain connection may also be involved in symptom generation and visceral hypersensitivity in IBS. It is important to note that symptoms such as pain are rather subjective and strongly influenced by psychological factors such as hypervigilance or dysfunctional cognitions $^{7,8}$. In the IBS population the prevalence of psychological co-morbidity is high: $20-40 \%$ have anxiety or depression. These conditions are known to affect pain perception $^{9}$. In our cohort, the hypersensitive IBS group did not show significantly increased anxiety or depressive symptoms over the normosensitive IBS group. Neither were differences observed between hyper and normosensitive patients with respect to mental or physical quality of life. However, hypersensitive IBS patients had significantly increased intake of SSRI medication compared to normosensitive IBS patients. 
Serotonin metabolism is altered in patients with $\mathrm{IBS}^{10}$, but data with respect to differences in hyper- versus normosensitive patients are lacking. SSRIs act on the serotonergic metabolism. By modulating the bioavailability of serotonin locally, pain perception may also be affected.

\section{Pathophysiology of visceral hypersensitivity}

Visceral hypersensitivity in IBS is a phenomenon that has been studied for more than four decades. Although its exact pathophysiology has not been unravelled, there are a number of factors closely associated with the presence of visceral hypersensitivity. For instance, alterations in barrier function have been proposed to contribute to visceral hypersensitivity.

Increased intestinal permeability is considered an early event that may facilitate sensitization of neurons by noxious substances crossing the intestinal membrane, contributing to allodynia and/or hyperalgesia in a subset of IBS patients. Furthermore, permeation of bacteria and bacterial products can result in immune activation and local (low-grade) intestinal inflammation as has been observed predominantly in IBS-D patients.

Physiologically, the epithelial barrier is maintained by tight junction protein complexes, which regulate intestinal permeability. Increased intestinal permeability may allow noxious substances to pass the epithelial barrier. These permeated substances may trigger enteric afferent neurons projecting to the CNS or induce local inflammatory and immune responses. In Chapter 5, we have systematically explored permeability of various regions within the gastrointestinal tract (gastroduodenal, small intestine and large intestine) in patients with different IBS-subtypes. Gastroduodenal and small intestinal permeability were found to be increased in patients with IBS-C and IBS-D subtypes. After correction for confounding factors (i.e psychological symptoms, lifestyle factors and medication) gastroduodenal permeability in IBS was no longer significantly different from healthy controls. In the small intestine, however, permeability remained significantly increased over controls in patients with IBS-D, even after correction for the aforementioned confounding factors. Colonic permeability was not affected in IBS. Although some reports have pointed to a direct relation between intestinal barrier (dys)function and abdominal symptoms (e.g. visceral sensitivity), in our study, we did not observe such a relation. In our study, we analyzed baseline permeability levels, without assessing the effect of individual stressors. Several stressors have shown to have an effect on intestinal permeability and tight junction expression per se. Stress for instance is an important trigger, which has been associated with altered permeability $^{11,12}$. Our findings do not preclude that IBS patients, especially those with hypersensitivity, have a decreased capacity to react adequately to stressors. Therefore, 
in future studies, it would be interesting to evaluate intestinal permeability in both hyper and normosensitive IBS patients.

In another study by our group, changes in small intestinal permeability were found to be related to serotonin metabolism and to visceral (hyper)sensitivity both in patients with IBS and healthy controls. In healthy subjects however, administration of the serononin precursor 5-hydroxytryptophan (5-HT) was found to enhance barrier function by up-regulating transcription for TJ proteins Zonula Occludens (ZO-1) and Occludin. In IBS, such increased transcription after 5-HT suppletion was not observed. The altered serotonergic regulation as observed in these IBS patients may therefore impair a potential protective effect to reinforce barrier function as seen in healthy subjects. This could suggest that in IBS, pain perception is affected by serotonin in a direct and indirect manner, i.e. as a result of altered permeability.

In Chapter 6, we further elaborated on potential mechanisms involved in altered intestinal barrier function in IBS-D. We found that plasma tryptase and LPS levels were increased in IBS patients and that in a newly established in vitro 3D model of the gut epithelial barrier, plasma from IBS-D patients was able to induce an increased permeability. In line with our previous in vivo results on permeability in IBS-D, and supported by data from Zhou et al. these data confirm that the IBS-D subtype may involve a different pathophysiology, compared to the other disease subtypes. When assessing in vivo intestinal permeability with multi-sugar tests, it is highly recommended that future studies in patients with IBS should always take into account the potential confounding factors.

Apart from intestinal permeability, also intestinal inflammation has been associated with visceral hypersensitivity. In this respect mast cell quantity and even more so, mast cell degranulation was found to be specific for IBS. Mast cells have also been found in closer proximity to enteric neurons. Inflammatory mediators, such as tryptase and histamine that are released by mast cells are able to trigger and sensitize enteric afferent neurons ${ }^{13,14}$. We have not evaluated mast-cell activity, but it could be interesting to further explore the contribution of mast cells to visceral hypersensitivity, also from a therapeutic standpoint.

Finally, increasing evidence demonstrates that the intestinal microbiota may also contribute to the pathophysiology of IBS. Findings in patients with IBS, show that the intestinal microbiota composition is different and less diverse compared to healthy subjects and may even differ between subgroups of IBS patients ${ }^{15,16}$. Alterations in the intestinal microbiota may result in altered epithelial barrier function and intestinal bacteria may even affect visceroperception directly ${ }^{17}$. Studies comparing the intestinal 
microbiota of hypersensitive versus normosensitive patients are lacking. However, evidence from animal models suggests that probiotic strains might modulate intestinal pain $^{18}$. Therefore, targeting the intestinal microbiota using probiotics or prebiotics may provide a strategy to improve symptoms associated with visceral hypersensitivity in IBS. Probiotics for instance, can directly affect visceroperception by interacting with the enteric nervous system, e.g. $\mu$-opoid receptors and cannabinoid receptors ${ }^{17}$, or indirectly by modulating intestinal permeability or immune status. In Chapter 7 we focused on IBS patients with visceral hypersensitivity and evaluated the effect of a multispecies probiotic on hypersensitivity and on daily symptoms. In a double-blind randomized controlled design, the multispecies probiotic was not found to be effective in decreasing visceral hypersensitivity or reducing IBS symptoms, when compared to placebo. The negative outcome may have resulted from several factors, among them the usage of a multi-strain instead of single strain probiotic. Nowadays, interest with respect to microbiota and metabolism is shifting from probiotics to prebiotics.

\section{Clinical relevance of visceral hypersensitivity}

Over the years, measuring visceroperception has been disputed and gastroenterologists and researchers have questioned the additional value of visceral hypersensitivity assessment. Not only are the underlying mechanisms leading to visceral hypersensitivity not completely understood, also is visceral hypersensitivity only present in part of the IBS patients, which makes it difficult to translate the present hallmark into a biomarker that is specific and ubiquitous for IBS. Furthermore, the prevalence varies substantially between studies and in this respect, internationally standardised procedures that assess visceral sensitivity are needed. Such standards allow comparison of prevalences of hypersensitivity among populations and between institutions. On the other hand, a characteristic that is present in over $50 \%$ of subjects with this very heterogenous disorder, is highly relevant and deserves further evaluation. Furthermore, there is substantial evidence that visceral hypersensitivity is associated with IBS symptomatology. To date, hypersensitive patients are distinct from normosensitive patients, with respect to symptom severity and presentation, and potentially also with respect to underlying pathophysiology. Assessment of visceral perception for research purposes allows further characterisation and phenotyping of the IBS population (i.e. in addition to subtyping based on bowel habits), which can be important for the development of new treatment strategies.

It is important that future translational research focuses on mechanisms relevant for subgroups within IBS, as well as on in vitro and ex vivo models that allow more high throughput evaluation. The 3D model that we have employed for the study in Chapter 6 is a suitable physiological model to investigate pathophysiological mechanisms at the level of the intestinal barrier. Furthermore, for both patients as well as clinicians, the concept of visceral hypersensitivity in the clinical setting provides a 
tool to a) explain the origin and severity of symptoms b) understand symptom generation c) for targeted therapeutic interventions d) self management of patients (diet, exclusion of provocative factors, relaxation training etcetera).

We think that it is of key importance to obtain further insight in pathophysiological mechanisms and ways of subtyping of patients with IBS. Although the Rome III criteria distinguish between subtypes based on predominant bowel habits, pathophysiological differences between subtypes are not clear. A different categorisation should therefore be considered, especially since pathophysiological mechanisms between current subtypes are overlapping (e.g. visceral hypersensitivity). Obtaining such insight will require large (international) cohorts, which not only standardize their phenotypical and genotypical characterization, but also extensively evaluate potential pathophysiological markers, using advanced modelling approaches. This may aid in identifying subgroups of IBS patients that could benefit from targeted interventions. 


\section{References}

1. $\mathrm{Ng} \mathrm{C}$, Malcolm A, Hansen R, Kellow JE. Distension technique influences the relationship between colonic and rectal hypersensitivity in irritable bowel syndrome. Neurogastroenterology and motility : the official journal of the European Gastrointestinal Motility Society. 2006;18:206-10.

2. Simren $M$, Abrahamsson $H$, Bjornsson ES. An exaggerated sensory component of the gastrocolonic response in patients with irritable bowel syndrome. Gut. 2001;48:20-7.

3. van der Veek PP, Van Rood YR, Masclee AA. Symptom severity but not psychopathology predicts visceral hypersensitivity in irritable bowel syndrome. Clin Gastroenterol Hepatol. 2008;6:321-8.

4. Mertz H, Naliboff B, Munakata J, Niazi N, Mayer EA. Altered rectal perception is a biological marker of patients with irritable bowel syndrome. Gastroenterology. 1995;109:40-52.

5. Naliboff BD, Berman S, Chang L, Derbyshire SW, Suyenobu B, Vogt BA, Mandelkern M, Mayer EA. Sexrelated differences in IBS patients: central processing of visceral stimuli. Gastroenterology. 2003;124: 1738-47.

6. Chang L, Mayer EA, Labus JS, Schmulson M, Lee OY, Olivas TI, Stains J, Naliboff BD. Effect of sex on perception of rectosigmoid stimuli in irritable bowel syndrome. Am J Physiol Regul Integr Comp Physiol. 2006;291:R277-84.

7. Merskey H. BN. Classification of Chronic Pain, Second Edition, IASP Task Force on Taxonomy - Part III: Pain Terms, A Current List with Definitions and Notes on Usage. IASP. 1994:5.

8. Thijssen AY, Jonkers DM, Leue C, van der Veek PP, Vidakovic-Vukic M, van Rood YR, Clemens $\mathrm{CH}$, Masclee AA. Dysfunctional cognitions, anxiety and depression in irritable bowel syndrome. J Clin Gastroenterol. 2010;44:e236-41.

9. Dorn SD, Palsson OS, Thiwan SI, Kanazawa M, Clark WC, van Tilburg MA, Drossman DA, Scarlett $Y$, Levy RL, Ringel Y, Crowell MD, Olden KW, Whitehead WE. Increased colonic pain sensitivity in irritable bowel syndrome is the result of an increased tendency to report pain rather than increased neurosensory sensitivity. Gut. 2007;56:1202-9.

10. Spiller R. Serotonin and GI clinical disorders. Neuropharmacology. 2008;55:1072-80.

11. Snoek SA, Verstege MI, Boeckxstaens GE, van den Wijngaard RM, de Jonge WJ. The enteric nervous system as a regulator of intestinal epithelial barrier function in health and disease. Expert Rev Gastroenterol Hepatol. 2010;4:637-51.

12. Vanuytsel T, van Wanrooy S, Vanheel H, Vanormelingen C, Verschueren S, Houben E, Salim Rasoel S, Toth J, Holvoet L, Farre R, Van Oudenhove L, Boeckxstaens G, Verbeke K, Tack J. Psychological stress and corticotropin-releasing hormone increase intestinal permeability in humans by a mast celldependent mechanism. Gut. 2013 Oct 23.

13. Klooker TK, Braak B, Koopman KE, Welting O, Wouters MM, van der Heide S, Schemann M, Bischoff SC, van den Wijngaard RM, Boeckxstaens GE. The mast cell stabiliser ketotifen decreases visceral hypersensitivity and improves intestinal symptoms in patients with irritable bowel syndrome. Gut. 2010;59:1213-21.

14. Stanisor OI, van Diest SA, Yu Z, Welting O, Bekkali N, Shi J, de Jonge WJ, Boeckxstaens GE, van den Wijngaard RM. Stress-induced visceral hypersensitivity in maternally separated rats can be reversed by peripherally restricted histamine-1-receptor antagonists. PloS One. 2013;8:e66884.

15. Dai $\mathrm{C}$, Zheng $\mathrm{CQ}$, Jiang $\mathrm{M}, \mathrm{Ma} X Y$, Jiang $\mathrm{L}$. Probiotics and irritable bowel syndrome. World J Gastroenterol. 2013;19:5973-80.

16. Ohman L, Simren M. Intestinal microbiota and its role in irritable bowel syndrome (IBS). Curr Gastroenterol Rep. 2013;15:323.

17. Rousseaux C, Thuru X, Gelot A, Barnich N, Neut C, Dubuquoy L, Dubuquoy C, Merour E, Geboes K, Chamaillard M, Ouwehand A, Leyer G, Carcano D, Colombel JF, Ardid D, Desreumaux P. Lactobacillus acidophilus modulates intestinal pain and induces opioid and cannabinoid receptors. Nat Med. 2007; 13:35-7. 
18. Agostini S, Goubern M, Tondereau V, Salvador-Cartier C, Bezirard V, Leveque M, Keranen H, Theodorou V, Bourdu-Naturel S, Goupil-Feuillerat N, Legrain-Raspaud S, Eutamene H. A marketed fermented dairy product containing Bifidobacterium lactis CNCM I-2494 suppresses gut hypersensitivity and colonic barrier disruption induced by acute stress in rats. Neurogastroenterol Motil. 2012;24:376-e172. 
Summary 


\section{Summary}

Irritable bowel syndrome (IBS) is a frequently occurring gastrointestinal disorder in Western society with a heterogeneous disease phenotype. Its pathophysiology is incompletely understood and involvement of a multitude of mechanisms involved in the aetiology of this disorder has been proposed. Among these mechanisms, visceral hypersensitivity plays a prominent role as it is present in up to $60 \%$ of IBS patients and therefore considered a hallmark of IBS.

In Chapter $\mathbf{1}$ we briefly reviewed the recent literature with respect to IBS, its epidemiology and proposed pathophysiology and proposed the aims and outline of investigations.

Mechanical or chemical stimuli to the GI tract have been reported to trigger symptoms in patients with IBS. Especially food intake has been related to exacerbation of symptoms, such as abdominal pain. In Chapter $\mathbf{2}$ we investigated food-induced symptom aggravation, by measuring visceral hypersensitivity under fasted and postprandial circumstances. Aim was to optimise the rectal barostat procedure for the assessment of visceral hypersensitivity (in patients with IBS, compared to healthy control subjects (HC). Seventy-one IBS patients and $30 \mathrm{HC}$ underwent a rectal barostat procedure under fasting and postprandial conditions (liquid meal; $368 \mathrm{kCal} ; 19 \mathrm{gr}$ fat) and sensations of urge, discomfort and pain were scored on a visual analogue scale (VAS) over a pressure range of 0-50 $\mathrm{mmHg}$. Cut-off for hypersensitivity was based on previously defined criteria, i.e. mean pain threshold in HC minus 2SD (VAS >10 mm at pressure $23 \mathrm{mmHg}$ ). Post-prandially, IBS patients showed significantly increased VAS scores for al sensations. HC showed significantly increased postprandial scores for urge and pain only. Although intake of the liquid meal significantly increased pain perception, the number of patients with visceral hypersensitivity did not significantly differ before versus after meal intake ( $40.9 \%$ vs. $39.4 \% ; P=1)$. Meal intake therefore, did not increase the yield for the detection of visceral hypersensitivity. However, optimising the cut-off based on a large group of IBS patients may increase the accuracy to detect visceral hypersensitivity in these patients.

Hypersensitivity rates have been reported in literature to vary between $30 \%-90 \%$ and results are hard comparable between studies due to different barostat protocols applied and lack of clear cut-off values to appoint visceral hypersensitivity. To further optimise the barostat procedure in our research center, in Chapter 3 three cut-off criteria were compared based on their sensitivity and specificity to detect visceral hypersensitivity in 126 patients with IBS versus $30 \mathrm{HC}$. All patients and $\mathrm{HC}$ underwent a single barostat according to a semi-randomised staircase distension protocol and 
scored urge, discomfort and pain on a VAS. Cut-off was based either on 1) mean threshold for first pain sensation in HC minus 2 SD (i.e. VAS>10 at $23 \mathrm{mmHg}$ ) 2) $10^{\text {th }}$ percentile of the threshold for first pain sensation in $\mathrm{HC}$ or 3 ) maximum discriminative optimum between $\mathrm{HC}$ and IBS patients based on ROC curves for pain perception. Using these cut-offs resulted in $34.9 \%, 64.3 \%$ and $63.5 \%$ of all IBS patients being hypersensitive, respectively. The latter cut-off criterion had sensitivity of $63 \%$ and specificity of $90 \%$ and a positive predictive value of $96 \%$ hereby showing the best discriminative capacity to differentiate between patients with IBS and HC, based on rectal barostat distensions. Using this criterion, $63.5 \%$ of the IBS patients were found to be hypersensitive versus $10.0 \%$ of the $\mathrm{HC}$.

In Chapter 4. we aimed to unravel clinical and psychosocial characteristics associated with having visceral hypersensitivity. Using several validated questionnaires and a symptom diary, we examined clinical and demographic patient characteristics, psychological comorbidity (by HADS), the use of medication, symptoms (by GSRS) and quality of life (by RAND-36). The study population consisted of 188 IBS patients with $(\mathrm{N}=93)$ and without $(\mathrm{N}=95)$ visceral hypersensitivity, according to previously the defined criteria defined for our research institute (Chapter 3 ). Compared to the normosensitive patients, we found that having visceral hypersensitivity was significantly associated with younger age (mean \pm SEM: $36.9 \pm 1.62$ vs. $45.8 \pm 1.71$ years; $P<0.001$ ), female sex ( $81.7 \%$ vs. $66.0 \% ; P<0.05)$ and use of SSRI mediation ( $16.1 \%$ vs. $6.3 \% ; P<0.05)$. However, in the multivariate analysis only age remained significantly associated with having visceral hypersensitivity with an Odds Ratio of 0.97 (95\% Cl: 0.94; 0.99). In Apart of being significantly younger, the patients with hypersensitivity showed significantly increased scores for GSRS abdominal pain, indigestion, reflux and constipation syndrome. IBS-related symptom intensity and discomfort as well as mean symptom composite score were significantly increased in the hypersensitive patients. Future mechanistically oriented studies within these subgroups of patients may provide biological markers or predictors for patients with and without visceral hypersensitivity.

Chapter 5 explored intestinal barrier function, as being one of the mechanisms potentially involved in the aetiology of IBS and visceral hypersensitivity. Ninety-one patients with IBS and $94 \mathrm{HC}$ underwent a multi-sugar test for the assessment of intestinal barrier function per intestinal segment. Findings were corrected for potential confounding demographic factors (i.e. age, sex and BMI), psychological symptomatology (anxiety and depression, lifestyle factors (smoking and alcohol use) and the use of medication. Compared to HC, patients with IBS, unspecified for disease subtype, had significantly increased excretion of sucrose. However, after correction for confounders the sucrose excretion, indicating gastroduodenal permeability was no longer different between IBS patients and controls. With respect to small intestinal 
permeability we found that the patients with IBS-D had a significantly increased lactulose-rhamnose excretion versus HC $(0.023$ [0.0020-0.0999] vs. 0.014 [0.0002-0.1512]; $P<0.05)$, that remained statistically significant after correction for confounding factors. Colonic permeability was not different between IBS and controls. None of the IBS subtypes did show significant alterations in colon permeability. It was concluded that small intestinal permeability is significantly increased in patients with IBS-D, irrespective of confounding factors. The mechanisms underlying this increased permeability in IBS-D need to be further explored.. Our findings also demonstrated that potential confounders should be taken into account in future studies on intestinal permeability in IBS.

We further investigated potential local and systemic factors contributing to altered intestinal barrier function in patients with IBS (Chapter 6). Factors that have previously been shown to be able to modulate barrier function are tryptase and LPS. In order to assess the involvement of these mediators after basolateral exposure, intestinal barrier function was assessed in vitro using a 3D Caco-2 cell culture. Caco-2 spheroids were exposed to different concentrations of tryptase (10-50 $\mathrm{mU}$ ) and LPS (1-50 $\mathrm{ng} / \mathrm{ml})$, after which permeability was assessed using FITC dextran 4KD (FD4) permeation. Both tryptase $(20$ and $50 \mathrm{mU})$ and LPS $(6.25-50 \mathrm{ng} / \mathrm{ml})$ were found to significantly increase permeability of the 3D model (all $P<0.05$ ). Inhibition of these mediators using nafamostat mesylate for tryptase and polymyxin B for LPS significantly attenuated this effect $(P<0.01)$. Subsequently, the model was exposed to plasma samples of patients with IBS-D, IBS-C and HC from the basolateral side. Plasma of patients with IBS-D showed significantly increased levels of tryptase versus HC. Moreover, the plasma of IBS-D patients induced a significant increase in paracellular permeability, compared to IBS-C and HC. Selectively inhibiting tryptase and LPS significantly reduced the observed effect. These findings point to a role of systemic tryptase and LPS in epithelial barrier alterations in patients with IBS-D.

Targeting specific mechanisms in subgroups of IBS patients may improve treatment success. Accordingly, in Chapter 7, we performed a double blind randomised controlled intervention study in a group of 40 IBS patients characterised by a common pathophysiological mechanism i.e. visceral hypersensitivity. Patients were randomly assigned to either a specifically designed multispecies probiotic $(\mathrm{N}=21)$ or a placebo $(\mathrm{N}=19)$. Patients took their probiotic or placebo daily for a period of 6 weeks. Before and after the intervention, all patients underwent a rectal barostat procedure for the assessment of visceral pain perception. Secondary, they kept a symptom diary 2 weeks prior each barostat procedure. Although the percentage of patients with visceral hypersensitivity decreased significantly in the probiotic group (to $76.5 \% ; P<0.05$ ), it also did in the placebo group (to $71.4 \% ; P<0.05$ ) and did not differ between both groups. 
Improvement in pain scores and mean symptom scores also did not differ significantly between probiotic and placebo treated patients. We concluded that in our group of hypersensitive IBS patients, no significant effect of the multispecies probiotic could be observed. For future studies, targeting specific mechanisms using probiotics, treatment duration and probiotic product should be taken into account. 
Samenvatting 


\section{Samenvatting}

Het prikkelbare darm syndroom (irritable bowel syndrome - IBS) is een heterogene darmaandoening welke frequent voorkomt in de Westerse maatschappij. De pathofysiologie van IBS wordt nog onvolledig begrepen en een diversiteit aan biologische mechanismen wordt toegeschreven aan de etiologie van de aandoening. Een van deze mechanismen betreft viscerale hypersensitiviteit welke voorkomt in circa $60 \%$ van alle patiënten met IBS en als een typisch kenmerk van de aandoening wordt beschouwt.

In de algemene introductie (Hoofdstuk 1) wordt ingegaan op de recente literatuur en worden epidemiologie en pathofysiologie nader toegelicht. Tevens worden de hoofdlijnen van dit proefschrift aangegeven

Studies hebben reeds aangetoond dat de aanwezigheid van mechanische of chemische stimuli in het maag-darmkanaal symptomen kunnen induceren bij patiënten met IBS. Inname van voedsel bijvoorbeeld, is geassocieerd met klachtenverergering, onder andere van abdominale pijn. Daarom hebben wij in Hoofdstuk 2 deze voedselgeassocieerde symptoomverergering onderzocht, door de mate van viscerale sensitiviteit te meten onder nuchtere en postprandiale condities. Het doel hiervan was, om de rectale barostat procedure te optimaliseren teneinde visceral hypersensitiviteit te kunnen meten in patiënten met IBS. Hiertoe ondergingen 71 IBS patiënten en 30 gezonde vrijwilligers een rectale barostatprocedure onder nuchtere en postprandiale condities (vloeibare maaltijd; $368 \mathrm{kCal} ; 19 \mathrm{gr}$ vet). Tijdens de barostatprocedure werd de mate van aandrang, onaangenaam gevoel en pijn geschaald op een visueel analoog schalingsformulier (VAS) tijdens het toedienen van drukken variërend tussen $050 \mathrm{mmHg}$. De afkapwaarde voor viscerale hypersensitiviteit was de gemiddelde pijndrempel in gezonde vrijwilligers, minus 2 SD (VAS>10 mm bij een druk van $23 \mathrm{mmHg}$ ), conform eerder gedefinieerde criteria. De IBS patiënten scoorden postprandiaal alle sensaties significant hoger ten opzichte van de nuchtere toestand. De gezonde vrijwilligers scoorden enkel aandrang en pijn hoger na de maaltijd. Het aantal patiënten met viscerale hypersensitiviteit daarentegen, nam niet significant toe na inname van de maaltijd ( $40.9 \%$ vs. $39.4 \% ; P=1)$. Aan de hand van deze resultaten concludeerden we dan ook dat het innemen van een maaltijd voorafgaande aan de barostatprocedure niet bijdraagt aan het vergroten van de detectie van hypersensitiviteit. Echter, het optimaliseren van de afkapwaarde zou wel hieraan kunnen bijdragen.

Zoals in de literatuur beschreven, komt viscerale hypersensitiviteit voor bij 30-90\% van alle patiënten met IBS. Vanwege deze heterogeniteit in prevalentie, maar ook vanwege de verschillende barostatprotocollen en definities voor hypersensitiviteit die worden 
toegepast is het lastig om data van verschillende studies te vergelijken. In Hoofdstuk 3 hebben wij de barostatprocedure geoptimaliseerd voor ons onderzoekscentrum. Hiertoe zijn 3 criteria voor hypersensitiviteit met elkaar vergeleken aan de hand van de sensitiviteit en specificiteit om viscerale hypersensitiviteit te detecteren in 126 patienten met IBS versus 30 gezonde vrijwilligers. Alle patiënten en vrijwilligers hebben hiertoe een rectale barostat ondergaan conform een semi-gerandomiseerd trapsgewijs protocol waarbij zij aandrang, onaangenaam gevoel en pijn scoorden op een VAS. De afkapwaarden betroffen 1) gemiddelde drempel voor first pain in gezonde vrijwilligers minus 2SD (VAS>10 bij $23 \mathrm{mmHg}$ ) 2) het 10e percentiel van de pijndrempel voor first pain bij gezonde vrijwilligers of 3 ) het op ROC-curves gebaseerde onderscheidende optimum voor pijn scores tussen gezonde vrijwilligers en IBS patiënten. Aan de hand van deze afkapwaarden vonden wij dat respectievelijk $34.9 \%$, 64.3\% en $63.5 \%$ van de IBS patiënten viscerale hypersensitiviteit vertoonden. De laatste afkapwaarde toonde een sensitiviteit van $63 \%$ bij een specificiteit van $90 \%$ en een positief voorspellende waarde van $96 \%$ en was hiermee het best onderscheidend tussen patiënten met IBS en gezonde vrijwilligers op basis van rectale distensies. Gebruik makend van dit laatste criterium was $63.5 \%$ van de IBS patiënten en $10.0 \%$ van de gezonde vrijwilligers hypersensitief.

Om meer inzicht te krijgen in welke klinische en psychosociale kenmerken geassocieerd zouden zin met viscerale hypersensitiviteit, worden in Hoofdstuk 4 de klinische en demografische patiëntkenmerken, de psychologische comorbiditeit (HADS), medicatiegebruik, symptomen (GSRS) en kwaliteit van leven (RAND-36) vergeleken tussen 95 normo- en 93 hypersensitieve IBS patiënten (conform het criterium beschreven in Hoofdstuk 3). Onder de hypersensitieve patiënten was de leeftijd significant lager (gemiddelde \pm SEM: $36.9 \pm 1.62$ vs. $45.8 \pm 1.71$ jaren; $P<0.001$ ), waren meer patiënten van het vrouwelijk geslacht $(81.7 \%$ vs. $66.0 \% ; P<0.05)$ en meer SSRI gebruik $(16.1 \%$ vs. $6.3 \% ; P<0.05)$ in vergelijking tot de normosensiteve patienten. Echter, in de multivariate analyse was alleen nog leeftijd significant geassocieerd met het hebben van viscerale hypersensitiviteit (OR 0.97; 95\% Cl: 0.94; 0.99). Naast het hebben van een jongere leeftijd, toonden de hypersensitieve patiënten hogere scores op de GSRS voor pijn, indigestie, reflux en obstipatie-syndroom en voor IBSgerelateerde symptomen. Toekomstige mechanistische studies kunnen mogelijk ophelderen welke biologische markers of predictoren patiënten kunnen onderscheiden op basis van viscerale hypersensitiviteit.

In Hoodstuk $\mathbf{5}$ is de barrièrefunctie van de darm onderzocht als potentieel mechanisme, betrokken bij de etiologie van IBS en viscerale hypersensitiviteit. Middels een multi-suiker test, werden 91 patiënten met IBS en 94 gezonde vrijwilligers getest voor barrièrefunctie per darmsegment. De bevindingen werden gecorrigeerd voor 
potentiële confounders, te weten: demografische factoren, psychosociale symptomen (angst, depressie), leefstijl factoren (roken en alcoholgebruik) en medicatiegebruik. Patiënten met IBS toonden initieel een significant verhoogde sucrose excretie, indicatief voor een verhoogde gastro-duodenale permeabiliteit; dit verschil verdween na correctie voor confounders. We vonden verder dat patiënten met IBS-D een significant verhoogde lactulose-rhamnose excretie toonden versus gezonde vrijwilligers (0.023 [0.0020-0.0999] vs. 0.014 [0.0002-0.1512]; $P<0.05)$, ook na correctie voor confounders. Geen van de IBS-subtypes toonde een verschil in colon-permeabiliteit. Concluderend vonden we dat de dunne darmpermeabiliteit significant is toegenomen in patiënten met IBS-D, ongeacht de aanwezigheid van confounders. De mechanismen die hiertoe leiden dienen echter verder te worden onderzocht.

Daarom hebben wij in Hoofdstuk 6 onderzocht welke systemische factoren zouden kunnen bijdragen aan een verstoorde barrièrefunctie in patiënten met IBS. Studies hebben reeds aangetoond dat tryptase en LPS de darm barrièrefunctie kunnen beïnvloeden. In ons onderzoek hebben wij onderzocht wat het effect van deze mediatoren is op de darm barrière na basolaterale toediening in de in vitro situatie. Drie dimensionale (3D) spheroids, bestaande uit Caco-2 cellen werden basolateraal blootgesteld aan verschillende concentraties tryptase (10-50 mU) en LPS (1-50 ng/ml), waarna de FITC dextran 4KD (FD4) permeatie van de basolateraal naar de luminale zijde werd onderzocht. Zowel toediening van tryptase (20 en $50 \mathrm{mU}$ ) als LPS $(6.25-50 \mathrm{ng} / \mathrm{ml})$ resulteerde in een significante toename in basolaterale naar luminale FD4 flux van het 3D model $(P<0.01)$. Vervolgens werd het model blootgesteld aan plasma van patiënten met IBS-D, IBS-C en gezonde vrijwilligers, eveneens aan de basolaterale zijde. Het plasma van patiënten met IBS-D toonde allereerst een significant verhoogde concentratie aan tryptase versus gezonde vrijwilligers. Daarbij induceerde dit plasma ook een verhoogde basolaterale naar luminale FD4 flux in vergelijking tot het plasma van IBS-C en van de gezonde vrijwilligers. Het inhiberen van tryptase en LPS leidde vervolgens tot normalisatie van het effect. Middels dit onderzoek hebben wij aangetoond dat het systemische compartiment mogelijk bijdraagt aan barrièredysfunctie in patiënten met IBS-D.

Het focussen van interventies op pathofysiologische mechanismen binnen IBS kan mogelijk bijdragen aan verhoogde mate van succes bij interventies. Derhalve is in Hoofdstuk 7 specifiek gefocust op het mechanisme viscerale hypersensitiviteit. In een dubbel gerandomiseerde placebo-gecontroleerde interventie met 40 IBS patiënten, allen hypersensitief, hebben wij onderzocht wat het effect van een speciaal ontworpen multi-species probioticaproduct zou zijn op de darmgevoeligheid. De patiënten werden gerandomiseerd toegewezen aan het multi-species probioticaproduct of een placebo en dienden dit dagelijks in te nemen gedurende 6 weken. Voorafgaande en na afloop 
van de interventie ondergingen alle patiënten een rectale barostatprocedure om de viscerale sensitiviteit te evalueren. Het percentage patiënten met viscerale hypersensitiviteit daalde significant in de probioticagroep (naar $76.5 \% ; P<0.05$ ). Echter, een gelijksoortige daling vond ook plaats in de placebogroup (naar $71.4 \% ; P<0.05$ ). Daarbij was de mate van het verschil niet significant tussen de twee groepen, probiotica versus placebo. Wij concludeerden dat het multi-species probioticaproduct in onze groep van hypersensitieve IBS patiënten geen effect had. Met het oog op toekomstige probioticastudies is het van belang de interventie te richten op specifieke pathofysiologische mechanismen, alsook de duur van de behandeling in acht te nemen. 
Dankwoord 


\section{Dankwoord}

Alle begin is moeilijk.

Denk aan het schrijven van een proefschrift, of nog simpeler: een artikel. Of het begin van de eerste zin, of de keuze van juist dat woord waarmee die zin begint. Evenzo kan het kiezen voor een nieuwe opleiding of baan moeilijk zijn of misschien die baan zelf. Of het starten van je eigen onderneming....

Misschien is beginnen zelfs wel moeilijker dan afsluiten. Hoewel het afronden van een proefschrift ook niet altijd makkelijk is. Maar het biedt kansen. Zo gaat het afsluiten van het één vaak gepaard met de start van het ander.

En om te kunnen afronden, maar ook om vol overtuiging te kunnen beginnen geldt dat volharding een absolute vereiste is. Maar minstens evenveel de steun die ons geboden wordt door collega's, vrienden en familie. Want zoals een Afrikaans gezegde luidt: 'If you want to go fast, go alone. If you want to go far, go together'.

Er zijn te veel mensen die een rol spelen of hebben gespeeld in het afronden van mijn proefschrift. Een aantal wil ik in dit dankwoord specifiek benoemen; het is nu mijn beurt om jullie te bedanken.

Een tweetal mensen die ik specifiek wil bedanken hebben een belangrijke rol gespeeld in de opvoeding van mij, Khaya en Nandi. Onze jeugdjaren hebben we samen onder een dak gewoond. Jullie waren als een soort van tweede ouders voor ons. Ik vind het daarom ook erg jammer dat jullie deze feestelijke dag niet meer hebben mogen meemaken. Al was ik er lang van overtuigd dat u op de dag van de verdediging ooit op de voorste rij zou komen te zitten oma! Dat u dan trots geweest zou zijn, dat weet ik zeker!

Mama en Papa, jullie hebben mij gevormd tot wie ik ben. Ik waardeer jullie continue steun en begrip jegens mij, maar ook naar Khaya en Nandi. Ik weet dat jullie trots op ons zijn, maar wij zijn minstens zo trots op jullie. Ik weet van mezelf dat ik er nog lang niet ben en er is nog veel ruimte voor groei en verbetering, maar jullie taak zit er op nu is het aan mijzelf ;-). Mam, de hubs 't good gedaon, baeter haeij neet gekoos. Pap, ndiyabulela ukuba usoloko ungumzeleko omhle kum. Ik hou van jullie, you're the best parents ever! 
Khaya, er is veel veranderd in de afgelopen jaren. Ik wil je bedanken voor al het plezier dat we hebben beleefd toen we nog klein waren en op één kamer sliepen. Toen was je m'n kleine broertje, nu ben jij een van mijn belangrijkste raadgevers. Hoe snel kan de tijd gaan. Ik wil je specifiek bedanken voor het werk dat je hebt verricht in de opstartfase van mijn bedrijf en alle tijd die jij hierin hebt gestoken. Ik heb me wel eens zorgen gemaakt over je toekomst, maar je hebt je voor mij nu al meer dan bewezen! Jij beschikt over inzichten waarover niemand anders beschikt. Je gelooft ook in jezelf en dat maakt je sterk en goed in wat je doet. Je gaat het nog ver schoppen met je eigenwijsheid, ik geloof voor de volle $100 \%$ in je: maak er wat van lief broertje! 0 ja, ik hoop dat we de COMPOSITRON-X 3D toch echt gauw mogen realiseren ;-).

Nandisa, ook over jou heb ik me als grote broer wel eens zorgen gemaakt. Heel lang heb je niet geweten wat je wilde, wat je gelukkig zou maken, maar nu weet ik zeker dat het goed zit. Omdat jij dat ook weet! Ik heb inmiddels al veel van je werk gezien en dat is beter dan goed! Als ook hierop de regel 'streef altijd naar verbetering' van toepassing is, dan ga je het nog ver schoppen! Geen wonder dat we ondertussen ook professioneel samenwerkingspartners zijn - dat was vroeger wel anders he ;-) - want ik wil alleen het beste van het beste en dat ben jij! Bedankt voor hetgeen je tot dusver voor me hebt betekend, ik hoop dat we nog heel veel samen mogen doen! Ik hou van je lief zusje!

Lieve Helen, bedankt dat je in me gelooft. Wat heb ik het toch getroffen met een heerlijke meid zoals jij, die in haar ogenschijnlijke bescheidenheid net zo vurig en passievol kan zijn als ik, een tikkeltje minder tegendraads weliswaar, maar des te liever. Een mooier en completer persoon ken ik niet. Jij hebt me weten te temmen en bent de stabiele factor in mijn leven geworden. En wie had dat gedacht: ik en stabiliteit (had je het van je zelf wel gedacht 2 jaar geleden)? In een korte tijd heb ik dankzij jou enorm veel geleerd. Veel over zakelijke aangelegenheden, relaties en praten, over mensen, over mezelf. Ook over het leven delen met iemand waarvan je houdt. $\mathrm{O}$ ja, en over hoe vrouwen denken over trouwen. Maar daarvoor wil ik je toch nog een keer meenemen terug in de tijd. Naar Parijs, onder de Eiffeltoren... flauw van me he! Ik dank je specifiek voor de manier waarop jij mij hebt verrijkt. Ik weet zeker dat ook hier het Afrikaanse gezegde weer geldt. Helen, ik hou van je!

Mijn vrienden dank ik. Stuk voor stuk hebben wij elkaar gevormd en verrijkt. Een ieder heeft zijn of haar persoonlijke succes te danken doordat hij of zij onderdeel was van het geheel. Sander, die zondagavonden zullen me nog lang bijblijven. En vooral die ene specifieke uitspraak. Ik ben blij dat er mensen zoals jij zijn die begrijpen dat er meer in het leven is dan werk en dat ook waarderen. Bedankt, dat je al zo lang mijn vriend bent; dat dit zo moge blijven! 
Marcel, Gijs, Stéphanie, bedankt voor de continue interesse en voor de steun die jullie mij bieden in mijn bedrijf. Ik waardeer hetgeen jullie voor me hebben gedaan enorm.

Sjoerd en Richard bedankt voor jullie continue bereidheid me verder te helpen en jullie onmenselijke beschikbaarheid en responsiesnelheid waarmee jullie altijd reageren op weer een nieuw verzoek van me. Beiden hebben jullie een mooi staaltje vakwerk geleverd. Richard: jouw cover-design is überStark!

Verder wil ik al mijn oud-collega's van de MDL bedanken en in het bijzonder mijn paranimfen, mijn vrienden Daniël en Mark.

Daniël Keszthelyi. Wat ben ik blij dat er meer mensen zijn met een ongebruikelijke, onuitspreekbare naam! Partners in crime waren we. Op het werk en daarbuiten hebben we veel grappige en vooral ook mooie momenten beleefd. Nu geen partners in crime meer, kindjes krijgen (Daniël) en verstandiger worden (Samefko, Daniël?) zijn dan ook serieuze aangelegenheden. Bedankt dat je me hebt geïnspireerd, gestimuleerd en hebt mede-gevormd tot wie ik ben geworden. Ik voel me vereerd dat het avontuur dat ik met jou begon, ook samen met jou - aan mijn zijde als paranimf - afgesloten mag worden. Ik wens jou heel veel geluk met Rilana en Aeneas. Geniet van jullie mooie Valentijn en dat onze vriendschap nog lang moge duren!

Mark van Avesaat. Op jou kan ik altijd bouwen. Jij staat voor zekerheid. Dat bleek ook toen ik besloot de academie te verlaten. Je was het er niet mee eens, maar je steunde mij en mijn ideeën door en door, omdat jij wist waar mijn passie lag. Nog altijd ben ook jij een van mijn zakelijke adviseurs waarvoor ik je wil bedanken. Bedankt ook voor onze vriendschap, welke me veel waard is. Ik geloof dat jij getalenteerd bent en de carrière die je ambieert jou op het lijf geschreven is, bekwaam als arts, doch sensitief als persoon. Je bent een goed mens Mark!

Faro. Nu ben jij aan de beurt. Een echte collega ben je eigenlijk nooit geweest; net als die twee hierboven ben ook jij een echte vriend. We hebben nooit op dezelfde afdeling gewerkt en ook nu in het echte leven zijn onze vakgebieden zo enorm verschillend. Toch denken we over veel hetzelfde.

Jij was een van de weinigen die het niet alleen begreep, maar ook voelde wat het was om onderzoek te doen aan een Universiteit. We hebben veel steun aan elkaar gehad en 's levens hoogte- en dieptepunten vaak samen geëvalueerd. We analyseerden elke situatie en schatten kansen in op basis van mogelijkheden. Elk gesprek voelde bijna als een filosofisch leermoment, hoe kort het ook was. Daarom genoot ik altijd zo van die koffiemomenten met jou. En nog steeds, nu niet meer in het keukentje op de 3e of 5e 
verdieping, maar bij ons thuis in ons Maastrichtse stadstuintje, of soms bij jou in Amsterdam. Het fundament van onze vriendschap is onverwoestbaar, bedankt Faro!

Elhaseen, we made lots of fun. You were always in for a chat and a good cup of coffee, of course never without the sweets! The 3D model is your pride of which you thought me a lot in the laboratory. Doing in vitro work and working with cells is so time consuming. Together we often killed time in the lab or behind the confocal microscope by extensively evaluating my weekend adventures or the difficulties that they had resulted in... You are a funny guy Elhaseen and that's why I loved working with your. Thanks for making the lab a little bit more human-like by bringing your joy and pleasure to that boring place. I hope you and your family do well!

Zlatan, nog immer waardeer ik de koffiemomenten gedurende de laatste periode van mijn aio-schap. Die zomer van 2013 dat we een maand lang werkten van 07:30 - 21:00 om de laatste loodjes van mijn werk afgerond te krijgen. Bedankt voor je hulp toen en in de afgelopen maand bij het voorbereiden van mijn verdediging. Het was een uiterst prettige samenwerking. Je bent een aangenaam gezelschap, oprecht en zeer gedreven.

Verder dank ik Freddy. Jouw opmerking: "Je collega's zijn minstens net zo belangrijk als het inhoudelijke werk" heeft me tijdens mijn studententijd tot nadenken aangezet om mijn academische carrière als promovendus voort te zetten. Met alle gevolgen van dien. Positieve gevolgen, waarvoor dank!

Martine, dank voor de rust die je uitstraalde en het geduld dat je toonde. Ik vond het fijn dat ik ook af en toe bij jou mijn hart kon en mocht luchten. Succes met je eerste halve marathon!

Annemieke, Bas, Bouke, Chantal, Elhaseen, Eveline, Fedde, Harm-Jan, Karen, Kirsten \& Kirsten, Mariëlle, Montserrat, Pee-Wee, Stevie, Steven, Tim, Zlatan. Ik bedank jullie voor de leuke tijd die jullie me hebben bezorgd tussen 2009 - 2013. We hebben een aantal (te) gekke uitstapjes beleefd. Ik heb een mooie tijd met jullie gehad, dankjewel!

Al mijn stagiaires, dank voor jullie ondersteuning: Inge Werter; Ronald - Sliepe Sleijpen; Mark - de Lange - van Avesaat; Gwen Dackus; Nicole - Blondie - Oldenstam; Stefan - Flörkemeißter - Flörkemeier; Tiny Castermans; Vicky Weel en Ruud Verhees.

Tiny, bedankt voor de voortvarendheid waarmee je het afronden van mijn promotie in een stroomversnelling hebt doen komen. Ik weet zeker dat ik niet de gemakkelijkste ben geweest voor je, maar je hebt mij het leven in ieder geval een heel stuk 
gemakkelijker gemaakt in de laatste maanden. Mietsie, Elly, al liet hij ons een uur wachten, dankzij jullie was het altijd gezellig!

Nicole, Ria, Pierre, we hadden een prettige en zorgeloze samenwerking. Bij jullie op de functiekamer was het door alle drukte soms chaotisch, doch altijd heerste er een gemoedelijke sfeer. Bedankt voor alle wijze levenslessen Ria!

José, co-promotor, bedankt voor de aangename samenwerking. Je hebt me vaak laten lachen in je zoektocht naar elektronische vernieuwing en en je continue strijd met de IT-afdeling van het MUMC+.

Ad, $u$ was als een academisch vader-figuur voor me de afgelopen 5 jaar. $U$ bent er verantwoordelijk voor dat er - om met uw eigen woorden te spreken - "schot in de zaak" heeft gezeten. En dat ik uiteindelijk "in the flow" ben geraakt, vooral tegen het einde. Een flow overigens, waar ik nooit meer uit ben gekomen, allereerst dank daarvoor!

Communiceren via de e-mail deed $u$ altijd op een zeer eigenaardige wijze: Kort, kordaat en emotie-loos, bijna robot-achtig. Ik moet $\mathrm{u}$ eerlijk bekennen dat ik hier in het begin wel eens moeite mee heb gehad, maar later realiseerde ik me dat $u$ 1. niet van de digitale generatie bent (hoewel $u$ in korte tijd een behoorlijke inhaalslag heeft gemaakt) en 2. dit voor $u$ als drukbezet 'CEO' een zeer efficiënte en snelle manier was om te communiceren. Ik heb me overigens af gevraagd hoe dat zou gaan, nu ook de smartphone bij u z'n intrede heeft gedaan. Deze vraag werd nog voor het afronden van dit dankwoord beantwoord, namelijk toen ik u onlangs een adres door what's-appte: "Dank Gr ad".

Toen ik $u$ onlangs mailde, om advies te vragen en gebruik te maken van uw zakelijke inzichten, reageerde u juist niet als vanouds: "Samefko : Natuurlijk wil ik meedenken, bel mietsie naar om iets af te spreken... gr ad". Zoals een echte mens-vader betaamt was u meer dan bereid me verder te helpen in mijn vraagstuk. Ik weet zeker dat dit gesprek heeft bijgedragen aan het verstevigen van mijn fundering met het oog op mijn toekomst; ook hiervoor dank. De mail was overigens wel nog met alle 'spatie-kommaspaties' en n-m vervormingen inbegrepen. Wij onderzoekers hebben hier vaak plezier aan beleefd. Ik mis het eigenlijk wel een beetje.

Beste $\mathrm{Ad}$, ik ben $\mathrm{u}$ enorm dankbaar voor de kans die u mij geboden heeft mijzelf te verrijken. De jaren die ik als onderzoeker onder uw hoede heb mogen werken waren 4 hele mooie jaren. Ik heb genoten, we'll stay in touch!

Tot slot Daisy. Al een jaar lang mis ik onze fijne koffie-bijpraat momentjes: even lekker het verstand op nul alvorens de dag begon. Gauw een kamer verder binnenlopen en bijkletsen. Soms een beetje roddelen en dan weer over tot de orde van de dag. Je hebt 
me het belang doen inzien van het behalen van mijn doctorstitel, met name met het oog op mijn toekomst. De afgelopen 5 jaren waren niet altijd even makkelijk. Jij was degene en de enige die me heeft weten te motiveren en ervoor gezorgd dat de laatste punt in dit boekje uiteindelijk is gezet. Ik vond het overigens wel grappig hoe je me elke keer opnieuw weer overtuigde. Door telkens hetzelfde te zeggen: "...het veult straks auk fijn es ge det weer vaan ug taofel haejt..." en "...det kump waal goodj...". Maar ik wist dat het waar was.

Bedankt dat je dit hele proces als co-promotor in goede banen hebt geleid en als coach tot het eind hebt begeleid. Het behalen van deze titel is mij enorm veel waard en zonder jou zou dit geenszins realiteit zijn geweest. De champagne was heerlijk, ik kom gauw mijn schuld aflossen, Daisy, bedankt!

Lieve mensen, ik heb genoten van deze mooie (verlengde studenten)tijd. Het ga jullie allemaal goed. Maak er een feest van, want dat is het leven, maar wees zuinig op je lijf en geniet van je gezondheid!

Samefko 
Curriculum vitae 


\section{Curriculum vitae}

Samefko Ludidi was born on the 18th of February 1985 in Geleen, the Netherlands, to a Dutch mother and a South African father. In 2005 he started studying Health Sciences for which he obtained both, his Bachelor's degree in Human Movement Sciences and his Master's degree in Metabolism and Nutrition in 2009. His Master's thesis, which he wrote under supervision of prof. dr. Ad Masclee was entitled: "Serotonergic modulation of the intestine"

In September 2009, Samefko was appointed as a full-time Ph.D. student at the department of Internal Medicine, division of Gastroenterology and Hepatology at the Maastricht University Medical Centert, under supervision of his previous mentor, prof. dr. Ad Masclee and dr. Daisy Jonkers and dr. José Conchillo.

In August 2012, Samefko founded his company, in which he - as a part-time entrepreneur - implemented personalized health promotion programs. As of January 2014 Samefko has been working as a Scientific Health Consultant in the renamed company Samefko - Health by Science. Samefko pursues his career in implementing science-based personalised and large-scale health interventions and disease prevention programs. 
Scientific publications 


\section{Scientific publications}

Ludidi S, Jonkers DM, Elamin EE, Pieters HJ, Schaepkens E, Bours P, Kruimel J, Conchillo $J M$, Masclee AA. The intestinal barrier in irritable bowel syndrome: subtype-specific effects of the systemic compartment in an in vitro model. Submitted

Mujagic Z*, Ludidi S*, Keszthelyi D, Hesselink MA, Kruimel JW, Lenaerts K, Hanssen NM, Conchillo JM, Jonkers DM, Masclee AA. Small intestinal permeability is increased in diarrhoea predominant IBS, while alterations in gastroduodenal permeability in all IBS subtypes are largely attributable to confounders. Aliment Pharmacol Ther. 2014 Jun [Epub ahead of print]

Ludidi S*, Mujagic Z*, Jonkers D, Keszthelyi D, Hesselink M, Kruimel J, Conchillo J, Masclee A. Markers for visceral hypersensitivity in patients with irritable bowel syndrome. Neurogastroenterol Motil. 2014 Jun [Epub ahead of print]

Ludidi S, Jonkers DM, Koning CJ, Kruimel JW, Mulder L, van der Vaart IB, Conchillo JM, Masclee AA. Randomized clinical trial on the effect of a multispecies probiotic on visceroperception in hypersensitive IBS patients. Neurogastroenterol Motil. 2014;26:705-14

Keszthelyi D, Troost FJ, Jonkers DM, Helyes Z, Hamer HM, Ludidi S, Vanhoutvin S, Venema K, Dekker J, Szolcsányi J, Masclee AA. Alterations in mucosal neuropeptides in patients with irritable bowel syndrome and ulcerative colitis in remission: a role in pain symptom generation? Eur J Pain. 2013;17:1299-306

van der Schaar PJ, van Hoboken E, Ludidi S, Masclee AA. Effect of cholecystokinin on rectal motor and sensory function in patients with irritable bowel syndrome and healthy controls. Colorectal Dis. 2013;15:e29-34

Keszthelyi D, Troost FJ, Simrén M, Ludidi S, Kruimel JW, Conchillo JM, Masclee AA.Revisiting concepts of visceral nociception in irritable bowel syndrome. Eur J Pain. 2012;16:1444-54

Ludidi S, Conchillo JM, Keszthelyi D, Van Avesaat M, Kruimel JW, Jonkers DM, Masclee AA. Rectal hypersensitivity as hallmark for irritable bowel syndrome: defining the optimal cutoff. Neurogastroenterol Motil. 2012;24:729-33

Ludidi S, Conchillo JM, Keszthelyi D, Koning CJ, Vanhoutvin SA, Lindsey PJ, Leufkens AM, Kruimel JW, Jonkers DM, Masclee AA. Does meal ingestion enhance sensitivity of visceroperception assessment in irritable bowel syndrome? Neurogastroenterol Motil. 2012;24:47-53 
Valorisation 


\section{Valorisation addendum}

Medical knowledge has rapidly increased over the past decades. This reflected by the broad range of scientific research that is performed in the health sector nowadays. Also, the enormous output that is delivered and translated into scientific publications demonstrates the load of knowledge that we possess. At the same time, patients are better informed, supported by the digital era in which we thrive. It is therefore our duty to deliver high-quality research and healthcare, but also to create transparency that helps to justify our scientific decisions and to inform the general population adequately.

\section{Economic relevance}

Economic burden of chronic diseases is likely to increase, as a result of the increased expected lifespan, which is estimated to be up to 90 years in the year 2060 in the Netherlands. In addition, it is common knowledge that exposure to several (lifestyle) risk factors, for example reduced physical activity, the Western diet and air pollution is increasing and is associated with a broad range of diseases.

In the present thesis, we have evaluated clinical characteristics and potential underlying mechanisms in patients with irritable bowel syndrome (IBS) in order to obtain better insight in the pathophysiology of the this disorder, for which thus far no clearly effective therapy is available.

IBS is a highly prevalent heterogeneous disorder associated with abdominal pain, accompanied by altered bowel habits, for instance diarrhoea or constipation. It affects between $5-20 \%$ of the Dutch population, meaning that between $850,000-3,370,000$ people have symptoms I). of which the pathophysiology is incompletely understood and II). which are associated with a substantial disease burden. Compared to other chronic diseases with well-known pathophysiology (such as diabetes), patients with IBS report significantly decreased quality of life, both mentally and physically ${ }^{1,2}$ and also increased levels of cognitive dysfunctions, together leading to increased utilisation of health care ${ }^{3}$ and reduced work productivity ${ }^{4}$. Patients with IBS often state that they feel their condition is not well understood. In clinical practice this results in patients visiting several (different) specialists, or as they would describe it "being sent from pillar to post", without achieving real treatment success. Frequently occurring psychologic comorbidity amongst patients with IBS, not only adds to health care utilisation, it also necessitates support with psychologicals or behavioral/ psycho -therapy in addition to more conventional, often symptom-based treatment.

As a result, annual direct costs in the Netherlands are estimated to be $€ 1648$ per patient in primary care and $€ 2003$ in secondary care ${ }^{5}$. Although only about $30 \%$ of IBS patients consult a physician - of which $80 \%$ seeks help in primary health care and $20 \%$ is 
seen by a specialist in secondary health care ${ }^{5,6}$ - annual direct costs of IBS can be estimated to be at least $€ 741 \mathrm{mln}$.

Furthermore, indirect costs (in particular work absenteeism and reduced workproductivity) may comprise an even larger part of the economic burden for IBS. Therefore, not only for the sake of patients' health, but also for socio-economic reasons, further research in the field of IBS is highly needed to decrease disease burden. Below we will further elaborate on the relevance of the present thesis for its target population outside the research field and future innovation and potential products.

\section{Practical implications for patients and specialists}

So far, diagnosis is based on the Rome III criteria for IBS, for which exclusion of organic disease cause is required, followed by the presence of abdominal pain and altered defecation pattern for at least three months. A diagnostic approach thus, which is merely symptom-based.

Since altered visceral perception i.e. visceral hypersensitivity is one of the predominant symptoms present in about $50 \%$ of the IBS population, it is considered an important hallmark of the disorder. It may play a role in IBS pathophysiology as well and therefore using the rectal barostat as a procedure for assessing visceral sensitivity may aid in supporting the diagnosis of IBS.

The assessment procedure of visceral hypersensitivity by means of the barostat procedure is further optimised in the present thesis. Although direct consequences for daily clinical practice are not evident, patients being 'diagnosed' with visceral hypersensitivity often show mental relief. Apart of the scientific value in exploring IBS pathophysiology, the barostat procedure may help the patient learning to accept its bodily sensations when being found hypersensitive. The rectal barostat could therefore support disease coping, alongside with good doctor-to-patient communication. Therefore, it should be considered to use visceral hypersensitivity not only as an internal research-parameter, but also as a clinical outcome that can aid in explaining the disorder and may contribute to patient acceptance. For future studies, it would be interesting to investigate the impact of such measures on patient-related outcome measures, for instance quality of life and health care utilisation.

\section{Lifestyle factors: diet and funcional foods}

IBS patients themselves often associate food intake with symptoms. Surveys on perceived food intolerance find that over $60-90 \%$ of patients report their symptoms to be triggered by meals or specific food items, a hypothesis that is further studied in this thesis. Indeed, we found that meals high in fat can provoke symptoms. In particular, we 
demonstrated that the IBS patients showed increased symptomatology during a barostat procedure, when performed after consuming such a meal compared to fasting conditions. However, consuming a high-fat meal before the barostat did not improve the detection rate of visceral hypersensitivity in IBS. Which means that although from the patient's perspective meal intake increases symptoms, from a clinical perspective it does not have any added value in discriminating between subgroups of patients upon visceral perception (hypersensitve subgroup versus normosensitive subgroup) as a disease mechanism.

Instead of avoidance, patients also seek for means to actively reduce symptoms. Some have been under medication and many are exploring the field of altering their lifestyle to reduce symptoms by means of changing their diet or introducing functional foods. Although formally no legal definition exists, functional foods, are true foods that are ought to improve a person's health beyond basic nutrition. However, they are not considered to be medication. Being of such, probiotics have gained popularity and are available without a doctor's prescription. Also in the medical world, interest in probiotics and in the role of the intestinal microbiota, did result in a myriad of studies on their efficacy and potential mechanisms of action. It has been postulated that probiotics could act on several pathophysiological pathways that are related to IBS, to improve low-grade mucosal inflammation altered barrier function, and visceral hypersensitivity. However, overall findings of probiotic trials in IBS patients are not convincing. Most studies focused on the IBS population as a whole, without discriminating between groups upon potential pathophysiological differences. In this perspective, we therefore aimed to study a specific subgroup and one putative underlying mechanism: visceral hypersensitivity. Although we could not find a clear effect of a specifically designed multispecies probiotic on symptoms in patients with increased visceral perception, we believe that the approach to address specific disease mechanisms in IBS should be further investigated.

The fact that the pathophysiology of IBS is ought to be diverse, yet not wellunderstood, urges the necessity for more research at the field of functional gastrointestinal disorders. Bio-markers for IBS are thus far not yet available. Research is ongoing to unravel the potential of biological factors to provide us with markers for IBS, for instance derived blood, faeces or exhaled air. The heterogeneous nature and the fluctuating symptomatology over time however, makes it quite challenging to find such markers with a high disease-specificity. Therefore, it may be more promising to seek for markers that are either subtype-specific or related to pathophysiological mechanisms, such as hypersensitivity or altered intestinal permeability. Volatile organic compounds (VOC) for instance are already found to be able to discriminate between Inflammatory Bowel Diseases and healthy controls and preliminary data show 
promising results to diagnose IBS. In future, they may provide us with biological markers based on metabolic alterations related to host as well as microbial processes that can be detected in exhaled air. With very low patient-invasiveness, such markers may pave the way for developing interventions that truly focus on pathophysiology, rather than symptoms. 


\section{References}

1. El-Serag HB, Olden K, Bjorkman D. Health-related quality of life among persons with irritable bowel syndrome: a systematic review. Aliment Pharmacol Ther. 2002;16:1171-85.

2. Gralnek IM, Hays RD, Kilbourne A, Naliboff B, Mayer EA. The impact of irritable bowel syndrome on health-related quality of life. Gastroenterology. 2000;119:654-60.

3. Spiller R, Aziz Q, Creed F et al. Clinical Services Committee of the British Society of Gastroenterology. Guidelines on the irritable bowel syndrome: mechanisms and practical management. Gut 2007;56:1770-98.

4. Simrén M, Brazier J, Coremans G, Dapoigny M, Müller-Lissner SA, Pace F, Smout AJ, Stockbrügger RW, Vatn $\mathrm{MH}$, Whorwell PJ. Quality of life and illness costs in irritable bowel syndrome. Digestion. 2004;69(4):254-61. Epub 2004

5 Flik CE, Laan W, Smout AJ, de Wit NJ. Irritable bowel syndrome patients diagnosed in primary and secondary care; what explains the difference in health care costs. UEG13-ABS-4598

6 Flik CE, van Rood YR, Laan W, Smout AJ, Weusten BL, Whorwell PJ, de Wit NJ. A randomised controlled trial on hypnotherapy for irritable bowel syndrome: design and methodological challenges (the IMAGINE study).BMC Gastroenterol. 2011 11:137: 11-137 
